\title{
Silencing neural symphonies with deep brain stimulation
}

Citation for published version (APA):

van Zwieten, G. (2021). Silencing neural symphonies with deep brain stimulation. [Doctoral Thesis, Maastricht University]. Maastricht University. https://doi.org/10.26481/dis.20210709gz

Document status and date:

Published: 01/01/2021

DOI:

10.26481/dis.20210709gz

Document Version:

Publisher's PDF, also known as Version of record

\section{Please check the document version of this publication:}

- A submitted manuscript is the version of the article upon submission and before peer-review. There can be important differences between the submitted version and the official published version of record.

People interested in the research are advised to contact the author for the final version of the publication, or visit the DOI to the publisher's website.

- The final author version and the galley proof are versions of the publication after peer review.

- The final published version features the final layout of the paper including the volume, issue and page numbers.

Link to publication

\footnotetext{
General rights rights.

- You may freely distribute the URL identifying the publication in the public portal. please follow below link for the End User Agreement:

www.umlib.nl/taverne-license

Take down policy

If you believe that this document breaches copyright please contact us at:

repository@maastrichtuniversity.nl

providing details and we will investigate your claim.
}

Copyright and moral rights for the publications made accessible in the public portal are retained by the authors and/or other copyright owners and it is a condition of accessing publications that users recognise and abide by the legal requirements associated with these

- Users may download and print one copy of any publication from the public portal for the purpose of private study or research.

- You may not further distribute the material or use it for any profit-making activity or commercial gain

If the publication is distributed under the terms of Article $25 \mathrm{fa}$ of the Dutch Copyright Act, indicated by the "Taverne" license above, 


\section{GUSTA vaN ZWIETEN}

SLENATG

NEURAL SYMPHONIES

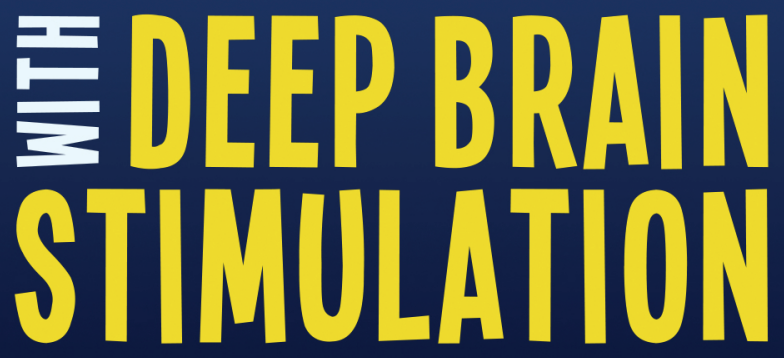





\section{Silencing neural symphonies with deep brain stimulation}


(C) Gusta van Zwieten, Maastricht 2021

All rights reserved. No part of this publication may be reproduced or transmitted in any form or by any means, without prior permission of the author or the publishers of the published chapters.

Cover design: Aksaramantra

Layout: Tiny Wouters

Printing: Ridderprint, www.ridderprint.nl

ISBN: 978-94-6416-542-5

Printing of this thesis was financially supported by $\mathrm{SBOH}$ and Maastricht University.

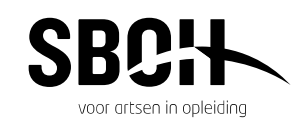




\title{
Silencing neural symphonies with deep brain stimulation
}

\author{
PROEFSCHRIFT
}

ter verkrijging van de graad van doctor aan de Universiteit Maastricht, op gezag van de Rector Magnificus, Prof. dr. Rianne M. Letschert volgens het besluit van het College van Decanen, in het openbaar te verdedigen op vrijdag 9 juli 2021 om 10:00 uur

door

Gusta van Zwieten 


\section{Promotores}

Prof. dr. Y. Temel

Prof. dr. R.J. Stokroos

\section{Copromotor}

Dr. M.L.F. Janssen

\section{Beoordelingscommissie}

Prof. dr. W.H. Mess (voorzitter)

Prof. dr. J.M.C. van Dijk (Rijksuniversiteit Groningen)

Prof. dr. B. Kremer

Dr. A. Mallinson (Vancouver General Hospital, Canada)

Dr. M. Moerel 
Voor mijn ouders 


\section{Contents}

$\begin{array}{ll}\text { Abbreviations } & 9\end{array}$

Chapter 1 General introduction

PART I Exploring deep brain stimulation in the auditory circuit

Chapter 2 Tinnitus: is there a place for brain stimulation?

Surgical Neurology International (2016)

Chapter 3 Alleviation of tinnitus with high frequency stimulation of the dorsal cochlear nucleus: a rodent study Trends in Hearing (2019)

Chapter 4 Inhibition of experimental tinnitus with high frequency stimulation of the rat medial geniculate body Neuromodulation (2019)

PART II Neuropathophysiological mechanisms

Chapter 5 Noise-induced neurophysiological alterations in the rat 85 medial geniculate body and thalamocortical desynchronization by deep brain stimulation Journal of Neurophysiology (2021)

Chapter 6 The effect of noise trauma and high frequency stimulation on thalamic sensory gating in rodents BioRxiv (2020)

PART III Clinical translation

Chapter 7 Deep brain stimulation for refractory tinnitus: a protocol for 131 a randomized double-blinded cross-over clinical pilot study Submitted

Chapter 8 General discussion 
Addendum

Summary

165

Nederlandse samenvatting

171

Impact paragraph

177

Dankwoord

183

Biography

191

List of publications

195 



\section{Abbreviations}

A1

ABI

ABR

AMI

Area LC

BAI

BBN

BDI-II

CI

$\mathrm{CN}$

$\mathrm{CV}$

DBS

DCN

DPSS

dwPLI

EEG

EP

EZM

fMRI

GPIAS

HADS

HFS

IC

LFP

LFS

LMEM

MGB

MRI

NAc

$\mathrm{OF}$

peSPL

SF-36

SFR

SG

tDCS

TENS
Primary auditory cortex

Auditory brainstem implant

Auditory brainstem response

Auditory midbrain implant

Locus of caudate neurons

Beck Anxiety Inventory

Broadband noise

Beck Depression Inventory II

Cochlear implant

Cochlear nucleus

Coefficient of variation

Deep brain stimulation

Dorsal cochlear nucleus

Discrete prolate spheroid sequences

debiased weighted phase lag index

Electroencephalography

Evoked potential

Elevated zero maze

functional magnetic resonance imaging

Gap-prepulse inhibition of the acoustic startle

Hospital anxiety and depression scale

High frequency stimulation

Inferior colliculus

Local field potential

Low frequency stimulation

Linear mixed-effects model

Medial geniculate body of the thalamus

Magnetic resonance imaging

Nucleus accumbens

Open field

peak equivalent sound pressure level

36-Item short form health survey

Spontaneous firing rate

Sensory gating

Transcranial direct current stimulation

Transcutaneous electric nerve stimulation 
TFI

TMS

TQ

VAS

VCN

VIM
Tinnitus functional index

Transcranial magnetic stimulation

Tinnitus questionnaire

Visual analogue scale

Ventral cochlear nucleus

Ventral intermediate nucleus of the thalamus 
General introduction

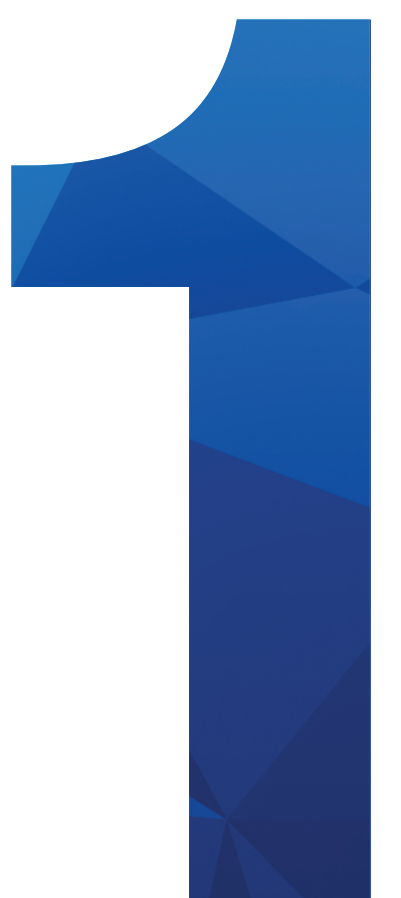





\section{Introduction}

Tinnitus originates from the Latin word tinnire, "to ring" and is commonly defined as the conscious perception of a sound in the absence of an external source. It is essential to realize that perception is different from sensation. Whereas sensation basically means the detection and processing of sensory information, perception includes interpretation and organization of this information. ${ }^{1}$

Two types of tinnitus can be distinguished. In objective tinnitus, a sound is generated in the body and conducted to the inner ear. Objective tinnitus usually has a vascular or muscular origin. This sound can often be detected by an external observer. Subjective tinnitus is the second type and much more common. Here, no detectable sound source is present and the sound can only be perceived by the patient who has tinnitus. ${ }^{2-4}$ This thesis focuses on subjective tinnitus only.

Subjective phenomena are often challenging to explain. Perhaps for this reason, wide-ranging ideas about tinnitus existed throughout history, influenced by cultural factors. Ancient Egyptians believed tinnitus occurred from a "bewitched ear", while Asian mysticism regarded tinnitus as "sensitivity to the divine". Probably a more modern-day view came from the Romans, who proposed the idea that tinnitus has a similar pathophysiology as seizures or depression. 3,4

Numerous historical figures are known to have suffered from tinnitus. Among many musicians and composers, Ludwig von Beethoven (1770-1827) suffered from progressive hearing loss and tinnitus. A different example is Charles Darwin (1809-1882), who kept daily records of the characteristics of his perceived tinnitus, which was possibly caused by Meniere's disease and/or loud sound exposure while hunting in his youth. ${ }^{3}$

The majority of people have experienced temporarily ringing in the ears, for example after visiting a concert. It has been shown that in the normal hearing population a transient phantom sound can be triggered in $80 \%$ of the people when placed in a soundproof room. ${ }^{5}$ Unfortunately, for $10-15 \%$ of the general population tinnitus is a chronic condition that requires medical evaluation. ${ }^{6}$ This makes this symptom one of the most common health problems. 
Comparable prevalence numbers are described in European countries, ${ }^{7,8}$ the USA, ${ }^{6,9}$ Japan, $^{10}$ and also lower income countries in the African continent ${ }^{11,12}$ as well as Asia. ${ }^{13}$ Most patients can function without limitations in daily life. Yet, one fifth of patients is more severely affected. It is estimated that in the Netherlands more than 500.000 adults suffer from severe tinnitus. ${ }^{14}$ Consequently, this places a high burden on society as well. This is illustrated by the high mean societal cost of illness of $€ 6.8$ billon in the Netherlands, of which the biggest part is not health care related. ${ }^{14}$ This is for example almost double as high as the total societal costs of low-back pain, which has been estimated to be $€ 3.5$ billion. ${ }^{15}$

Tinnitus is a heterogeneous phenomenon. Perceptual characteristics and different accompanying symptoms vary widely between patients, and frequently vary over time in each patient. The perceived sound can be a pure tone, noise-like or polyphonic sound, which can be present unilaterally, bilaterally or holocranially. Some patients hear it continuously and some only intermittently. ${ }^{16}$ Often, patients have hearing difficulties and hyperacusis. Many patients describe feelings of frustration, annoyance and irritability. In more severe cases, patients can suffer from anxiety, depression and insomnia and even cases of suicide have been described. It is intriguing that some patients suffer severely emotionally, while others are not much bothered by the phantom sound.7 Apparently, the severity of tinnitus is not determined by loudness, but by accompanying symptoms and impact on quality of life. ${ }^{17}$

The subjective nature of this disorder, in combination with the heterogeneity of the symptoms, and the accompanying and often disabling comorbidities, illustrate the complexity of this condition and its mechanisms. Therefore, research in the field of tinnitus is a great challenge.

\section{Tinnitus mechanisms}

Up to date, exact pathophysiological mechanisms are not understood. Many studies have been conducted in recent years, providing not only increasing insight in possible underlying mechanisms of tinnitus, but also basic audiological functioning. Here, I will discuss the most plausible concepts of the underlying mechanisms of tinnitus. This section is divided in four parts. The first part elaborates on the involvement of the cochlea in the development of 
tinnitus. After cochlear damage has occurred, neuronal changes appear in the central auditory pathway, which will be described in the second part. Thirdly, non-auditory networks will be discussed, as their involvement is essential for conscious perception of a (phantom) sound. Finally, I will discuss three proposed models underlying tinnitus development, namely central gain enhancement, thalamocortical dysrhythmia and breakdown of thalamic gating.

\section{Cochlea}

The cochlea is a spiral fluid-filled tube, located in the temporal bone. It is responsible for the transformation of pressure waves into electric signals. Sound pressure waves deform the basilar membrane in the area that is specific to the frequency of vibration. Here, stereocilia on the surface of inner hair cells bend and cause depolarization. Outer hair cells control and amplify the sensitivity of inner hair cells. Sensory information is transmitted to the brain via tonotopically organized auditory nerve fibers. ${ }^{18}$

The major risk factor for tinnitus development is hearing loss, such as presbyacusis, or hearing loss caused by noise exposure. ${ }^{19,20}$ As of now, it is thought that some degree of hearing impairment is required for tinnitus to develop. Even when elevated auditory thresholds do not occur, cochlear dead regions can still be found, ${ }^{21}$ as well as hair cell damage. ${ }^{22,23}$

Hearing loss or cochlear damage is a form of peripheral deafferentation. Typically, tinnitus patients judge their perceived tinnitus sounds as the same frequencies as their hearing loss regions. ${ }^{24}$ Comparisons between tinnitus and phantom pain have often been made. ${ }^{25}$ Peripheral deafferentation may produce plastic maladaptive compensatory changes in central brain regions, which can lead to phantom pain syndromes. A similar mechanism is postulated for phantom sound perception in tinnitus patients. ${ }^{26-28}$

\section{Central auditory pathway}

Processing of auditory information occurs within the brainstem, midbrain and cortex (Figure 1.1). Auditory nerve fibers originating from the cochlea synapse on neuronal cell bodies of the cochlear nucleus. This is the first station of the auditory pathway and consists of a dorsal and ventral part. Ventral neurons play a role in sound pattern identification while neurons of the dorsal division are important in sound localization. The vast majority of axons of cochlear 
nucleus cells cross over to the contralateral side of the brain. ${ }^{18}$ Fibers originating from the cochlear nucleus synapse in an area of the brainstem called the superior olivary complex, where information of both ears is incorporated. The inferior colliculus is the next station, which plays a major role in sound localization. Additionally, inputs from other sensory systems is incorporated for analysis of complex auditory scenes. ${ }^{29}$ Axons originating in the inferior colliculus project to the medial geniculate body (MGB) of the thalamus. This nucleus is a major relay and gateway between midbrain and cortex. Here, limbic and auditory information are integrated. Finally, signals arrive at the auditory cortex, where conscious awareness of (phantom) sounds might occur. This is dependent on aberrant neuronal activity within a broader cortical network involving frontal and parietal cortices as well as subcortical limbic regions. ${ }^{25}$

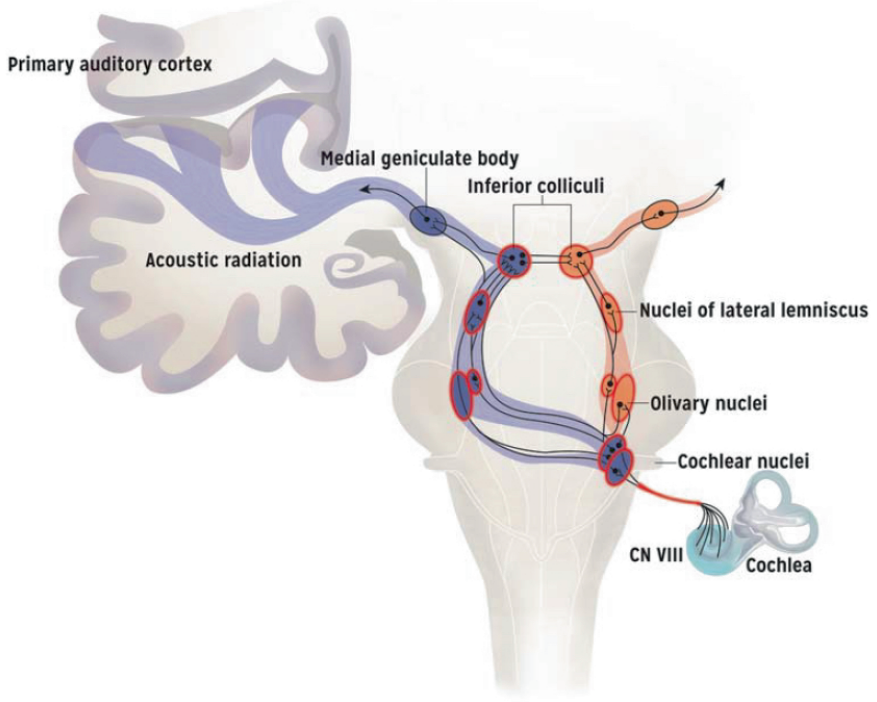

Figure 1.1 The auditory pathway from cochlea to auditory cortex. $\mathrm{CN}$, cranial nerve. By permission of Rammo, et al. 2018.30

In tinnitus, changes in neuronal activity are found throughout the central auditory pathway. The main neural correlates associated with tinnitus are hyperactivity, hypersynchrony, and tonotopic reorganization. ${ }^{26,31}$ 


\section{Nonauditory networks}

In recent years, there is growing evidence that nonauditory brain regions are involved in the perception and interpretation of sounds, and tinnitus in particular (Figure 1.2). It is well known that many factors can influence the way we perceive sounds, and tinnitus for that matter. To turn this around: tinnitus itself can have a certain influence on the same factors. Examples are attention, emotions, memory and sleep.

The fact that not all people who have hearing loss develop tinnitus, indicates that other top-down mechanisms in the brain are involved as well. According to one theory, tinnitus must occur from a deficient "noise-cancelling system", which mainly consists of nonauditory structures. 32,33

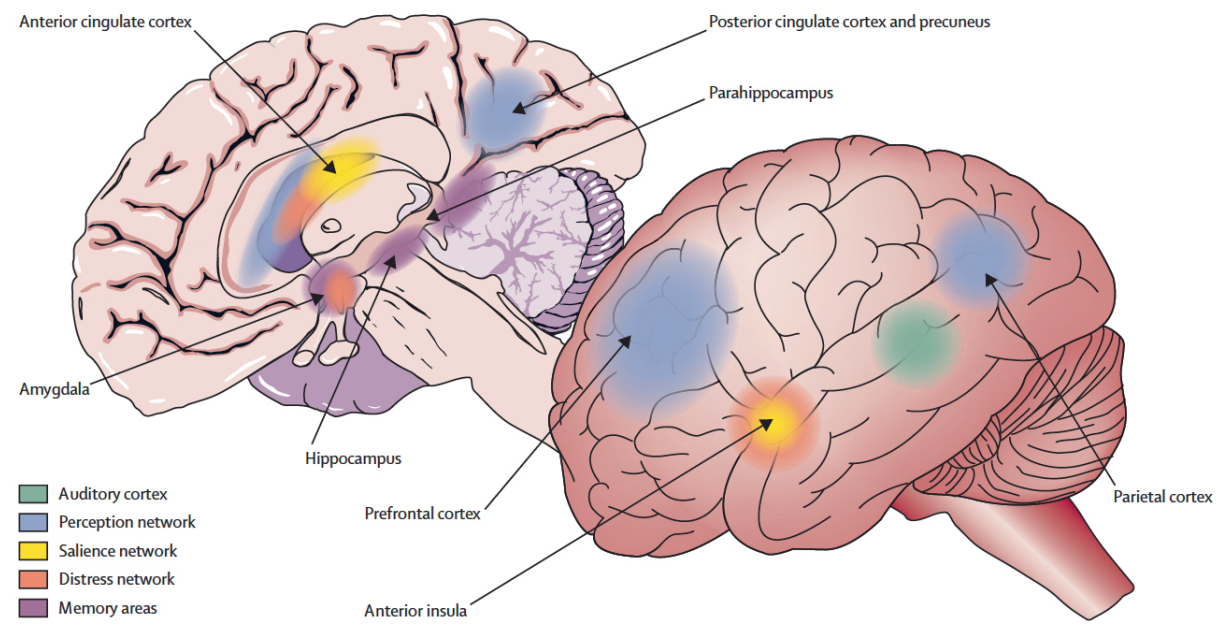

Figure 1.2 Brain networks involved in tinnitus perception. Involvement of the auditory cortex (green) alone is insufficient for tinnitus perception. For conscious awareness, coactivation of the perception network is needed, including the subgenual and dorsal anterior cingulate cortices, posterior cingulate cortex, precuneus, parietal cortex and prefrontal cortex (blue). Salience or attention to the phantom sound occurs when dorsal anterior cingulate cortex and anterior insula are activated (yellow). A distress network consists of the anterior cingulate cortex (orange), anterior insula, and amygdala. The parahippocampal area, amygdala, and hippocampus (purple) have a role in memory mechanisms and tinnitus. By permission of Langguth, et al. 2013.34 


\section{Tinnitus theories}

Neural plastic changes play a key role in tinnitus pathophysiology, but exact mechanisms remain controversial. Multiple models have been proposed.

\section{Central gain model}

According to this model, tinnitus is the result of a dynamic compensatory gain enhancement at many different levels of the central auditory system after sensory input loss. ${ }^{35}$ Electrophysiological studies have shown increased evoked responses following noise trauma. Possible underlying mechanisms might be a complex combination of: 1) decrease in inhibitory synaptic responses, 2) an increase in excitatory activity, and 3) alterations to intrinsic neuronal excitability. ${ }^{36}$

\section{Thalamocortical dysrhythmia}

Here, abnormal theta-range resonant interactions occur between thalamus and cortex, as a consequence of thalamic changes such as hyperpolarization due to deafferentation. In case of severe deafferentation, bursting theta activity acts as a carrier wave to access hippocampal memory processes. In a similar way, an increase in salience and attentional activity might be created. ${ }^{37,38}$

\section{Breakdown of thalamic gating}

According to this theory, tinnitus occurs if there is a failing "noise cancelling system" in addition to peripheral deafferentation. This system is an inhibitory feedback loop, consisting of paralimbic structures that connect to the thalamic reticular nucleus which in turns inhibits the MGB. If this thalamic gate fails, the tinnitus signal in the auditory pathway is relayed to the auditory cortex, where it leads to chronic tinnitus. ${ }^{32,39}$

To conclude, perception of tinnitus involves a large and complex interconnected network of neural structures. Multiple forms of tinnitus with various associated symptoms may be the result from dysfunction in different parts of the complex system. Involvement of the auditory pathway is mandatory for perception of both normal and phantom sounds.

Conscious regular hearing occurs when external noises induce harmoniously orchestrated neuronal activity and synchrony within and between the central auditory nuclei. In tinnitus, the same neuronal symphony can be observed, 
except without an external source, and in the absence of a conductor. In search of common grounds in the highly heterogenous group of tinnitus patients, we argue that the central auditory system is involved in all tinnitus patients. Therefore, we consider the central auditory pathway as the "core" of the complex tinnitus network.

\section{Clinical management}

During the last decades multiple therapies have been developed, some of them improved complaints in subgroups of tinnitus patients. Due to lack of consistent evidence, tinnitus care is still not standardized in many countries. ${ }^{40}$ There is no causal nor standard treatment. Nonetheless, this does not justify therapeutic nihilism. ${ }^{34}$ To arrange optimal clinical management, a multidisciplinary approach is essential.

According to Dutch and International guidelines, basic diagnostics and counselling is recommended for all patients. ${ }^{41,42}$ Diagnostics include an adequate history taking, assessing tinnitus severity, clinical ear examination, and audiological measurements of hearing function. Counseling is considered fundamental, even though the efficacy is undetermined. This form of psychoeducation assists patients to learn to cope with tinnitus and to achieve habituation to the sound.

In case of comorbidities, such as hearing loss or psychiatric conditions, further treatment should first focus on these. If hearing loss is more than $25 \mathrm{~dB}$, hearing aids are recommended. Even though hearing aids are widely prescribed, evidence from controlled trials is sparse. ${ }^{43,44}$ In case of severe tinnitus in combination with bilateral profound sensorineural hearing loss or unilateral profound deafness, cochlear implantation can be considered. The evidence for efficacy of this treatment is increasing, ${ }^{45}$ yet this treatment is only available for tinnitus patient with profound hearing loss. Anxiety or mood related disorders can be treated with psychological interventions and/or pharmaceutical therapy. ${ }^{42}$

If treatment of comorbidities is insufficient, symptom-oriented strategies should be considered. Cognitive behavioral therapy is advised in case of medium tinnitus severity. ${ }^{46}$ Here, the aim is to change maladaptive cognitive 
emotional and behavioral responses. It has been demonstrated that quality of life improves, even if tinnitus loudness itself does not change. Some patients find relieve from sound therapy. Even though acoustic stimulation is not actively advised in clinical guidelines because evidence is missing, it is commonly used in order to mask tinnitus; Apps and Youtube videos offering so called "tinnitus-relieving sounds" such as rainfall or white noise are readily available. Tinnitus retraining therapy is a specific form of sound therapy in combination with directive counseling and widely applied. One randomized controlled trial found considerable benefit, 47 but firm conclusions cannot be drawn due to the low quality of this study. ${ }^{48}$ Various other experimental sound paradigms, such as coordinated reset auditory stimulation, ${ }^{49}$ and musical therapy50,51 are still under research. Yet, convincing evidence for all these approaches is still lacking.52 Noteworthy, wide-ranging pharmacological therapies have been shown to be ineffective as a treatment for tinnitus loudness. Not a single compound has been approved by US Food and Drug Administration or European Medicines Agency for treatment of tinnitus. ${ }^{34}$

More recently, neuromodulation techniques obtained increasing interest in the treatment of tinnitus. In experimental clinical settings, noninvasive techniques such as repetitive transcranial magnetic stimulation and transcranial direct current stimulation are investigated. In some studies, subgroups of patients showed short lasting relieve, whilst others show negative results. ${ }^{53}$ Invasive methods of neuromodulation that have been investigated are auditory cortex stimulation and vagal nerve stimulation. At the moment, evidence does not support a place for such therapies in clinical practice. ${ }^{42,54}$ More prospective, randomized controlled trials are needed to assess effectiveness adequately.

\section{Deep brain stimulation}

Deep brain stimulation (DBS) is a form of minimally invasive neuromodulation. During a neurosurgical procedure, electrodes are implanted within a specific brain area. Thin subcutaneous wires connect these electrodes to an internal pulse generator, usually subcutaneously implanted underneath the clavicula or abdominal. Electrical impulses are produced and interfere with neuronal activity, which influence clinical symptoms. At the moment, DBS is an FDA approved treatment for several neurological disorders, such as Parkinson's disease and essential tremor (1997), dystonia (2003), obsessive- 
compulsive disorder (2009), and epilepsy (2018). The effect of DBS is being studied in clinical trials for Alzheimer's disease, chronic pain, major depression and other neurologic and psychiatric disorders. In the future, indications for DBS are likely to be expanding. ${ }^{55}$ Currently, more than 160.000 patients have already been treated.

A handful of human case reports and case studies have already shown that DBS can attenuate tinnitus. One case study described that conventional DBS surgery led to vascular injury in a specific part of the caudate nucleus ("area LC"), coincidentally causing tinnitus suppression. ${ }^{56}$ A following pilot study enrolled 6 patients with severe and chronic tinnitus who received DBS of the same nucleus. Results showed significant tinnitus suppression as measured with the TFI score (average reduction of 33\%) in 3 of 5 patients who fully completed the study, without induction of side-effects. ${ }^{57}$ In one other case report, researchers aimed to reduce the emotional content of tinnitus in one patient with DBS of the ventral anterior limb of the internal capsule. It has been demonstrated that DBS of this structure can improve mood and anxiety in major depressive disorder and obsessive-compulsive disorder. This patient eventually showed a reduction in TFI score of $63 \% .{ }^{58}$ Lastly, three out of seven patients who received DBS in nonauditory thalamus for movement disorders and also reported having tinnitus, reported reduced tinnitus loudness during stimulation. Even though these studies are small and the rationale for the chosen targets are often based on coincidental findings without much preclinical support, the results are encouraging.

DBS is widely applied, but exact working mechanisms are still under debate. DBS does not only influence the activity in the target nucleus, but in complete related networks. This is shown by evidence that DBS causes soma inhibition, as well as axonal activation. ${ }^{59}$ From clinical studies, we know that high frequency stimulation mimics a lesioning effect. This type of stimulation might disrupt abnormal neuronal activity and cause an informational-lesion within a network. ${ }^{60,61}$ In Parkinson's disease, it has been shown that high frequency DBS of the subthalamic nucleus can suppress hypersynchrony and bursting activity $^{62}$ and herewith the associated motor related symptoms of this disease. The same neural correlates have been described in tinnitus pathophysiology within the central auditory pathway. ${ }^{31}$ Therefore, we hypothesize that DBS of auditory structures can silence tinnitus with a similar mechanism. The three 
subcortical candidate structures of the core tinnitus network are the dorsal cochlear nucleus, inferior colliculus and MGB (Figure 1.3).

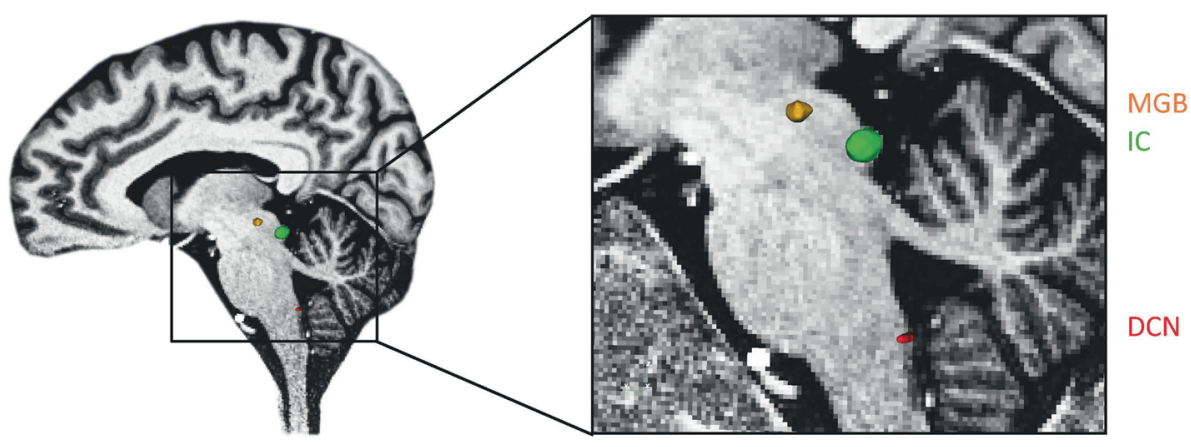

Figure 1.3 Sagittal MRI of human brain with three candidate targets for deep brain stimulation in tinnitus; dorsal cochlear nucleus (red), inferior colliculus (green), and MGB (orange).

\section{The problem}

Current treatment strategies aim at symptomatic relief, but still fail to eliminate the tinnitus percept effectively. Considering the existing theories on the pathophysiological mechanisms in tinnitus and the effect of DBS on neural disorders with aberrant neural functioning, we hypothesize that DBS has the potential to suppress tinnitus. Yet, it is unknown which structure should be targeted in human to treat tinnitus, what possible side-effects can be induced and which stimulation parameters are most effective. Furthermore, insufficient knowledge about the underlying neuropathophysiology hinders the development of treatment options. A better understanding of the neurophysiological origin of tinnitus is needed. This is a prerequisite for successful innovative neuromodulative therapeutic advances.

\section{A translational approach}

To challenge these difficulties, a well-considered translational neuroscientific approach is required. Hence, preclinical studies have been conducted. A validated noise-exposed tinnitus animal model was used. Here, rats were 
anesthetized and unilaterally exposed to a $115 \mathrm{~dB}$ loud 16-kHz octave banded noise for 90 minutes. Following noise-exposure, tinnitus can develop, as well as temporary hearing loss and hyperacusis. Tinnitus was assessed using the gapprepulse inhibition of acoustic startle response paradigm (GPIAS). This paradigm makes use of a diminished suppressing effect of the gap-prepulse on the acoustic startle response in tinnitus animals, as illustrated in Figure 1.4. Electrodes were implanted in specific brain targets using stereotaxy. ${ }^{63}$ This way, deep brain stimulation and tinnitus assessment could be applied in vivo.

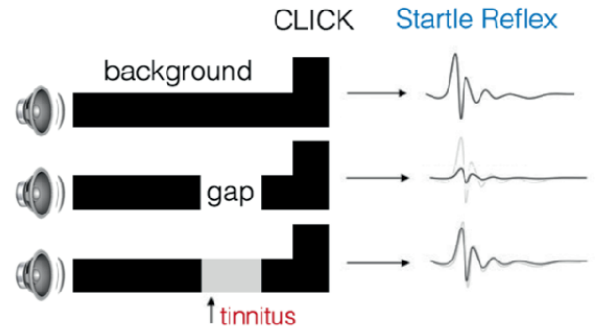

Figure 1.4 Principle of gap-prepulse inhibition of the acoustic startle response (GPIAS) for tinnitus assessment. In tinnitus animals, the suppressing effect of the pre-pulse gap on the startle response is smaller compared to control animals.

\section{Aim of this thesis}

In this thesis, we investigate whether DBS of auditory structures can be used to suppress tinnitus and what the mechanisms of action are. To reach this objective, the following studies have been conducted:

\section{Part I}

In Chapter 2, a review on the state-of-the-art of neuromodulation techniques to suppress tinnitus, with DBS in particular, will be presented. Next, results of two preclinical studies on the effect of DBS on tinnitus suppression will be discussed. Chapter 3 concerns a preclinical study on the effect of DBS of the dorsal cochlear nucleus on tinnitus. To elaborate on possible mechanisms underlying DBS and tinnitus, neuronal activity is assessed using an immediate early gene marker. In Chapter 4, the effect of DBS of the MGB in an animal model will be presented. Here, we assessed the effect of different stimulation paradigms on tinnitus behavior, and also evaluated potential side effects of stimulation. 


\section{Part II}

To further elaborate on underlying mechanisms of tinnitus and the effect of DBS, results of electrophysiological measurements will be discussed in this part. In Chapter 5 the effect of noise-trauma in rats on single unit activity in the MGB and effects of MGB DBS on local field potentials will be presented and discussed. Chapter 6 elaborates on the effect of noise-trauma and MGB DBS on sensory gating and auditory prediction.

\section{Part III}

The translation to clinical practice will be made in Chapter 7. Here, a clinical study protocol on the effect of DBS of the medial geniculate body in severe, refractory tinnitus patients will be presented. Finally, in Chapter 8 an overall discussion is provided. 


\section{References}

1. Freeman WJ. How brains make up their minds. Columbia University Press; 2000.

2. Møller AR. Hearing: Its Physiology and Pathophysiology. San Diego: Academic Press; 2000.

3. Dietrich S. Earliest historic reference of 'tinnitus' is controversial. J Laryngol Otol. 2004;118(7): 487-488.

4. Shaikh AG. A journey of tinnitus: myths, models, membranes and medicines. J Neurol Neurosurg Psychiatry. 2012;83(8):765-767.

5. Del Bo L, Forti S, Ambrosetti U, et al. Tinnitus aurium in persons with normal hearing: 55 years later. Otolaryngol Head Neck Surg. 2008;139(3):391-394.

6. Hoffman HJ, Reed GW. Epidemiology of Tinnitus. In: Snow JB, Jr., ed. Tinnitus: Theory and Management. BC Decker Inc; 2004.

7. Axelsson A, Ringdahl A. Tinnitus--a study of its prevalence and characteristics. Br J Audiol. 1989;23(1):53-62.

8. Krog NH, Engdahl B, Tambs K. The association between tinnitus and mental health in a general population sample: results from the HUNT Study. J Psychosom Res. 2010;69(3):289298.

9. Shargorodsky J, Curhan GC, Farwell WR. Prevalence and characteristics of tinnitus among US adults. Am J Med. 2010;123(8):711-718.

10. Michikawa T, Nishiwaki Y, Kikuchi Y, et al. Prevalence and factors associated with tinnitus: a community-based study of Japanese elders. J Epidemiol. 2010;20(4):271-276.

11. Khedr EM, Ahmed MA, Shawky OA, Mohamed ES, El Attar GS, Mohammad KA. Epidemiological study of chronic tinnitus in Assiut, Egypt. Neuroepidemiology. 2010;35(1):45-52.

12. Lasisi AO, Abiona T, Gureje O. Tinnitus in the elderly: Profile, correlates, and impact in the Nigerian Study of Ageing. Otolaryngol Head Neck Surg. 2010;143(4):510-515.

13. Xu X, Bu X, Zhou L, Xing G, Liu C, Wang D. An epidemiologic study of tinnitus in a population in Jiangsu Province, China. J Am Acad Audiol. 2011;22(9):578-585.

14. Maes IH, Cima RF, Vlaeyen JW, Anteunis LJ, Joore MA. Tinnitus: a cost study. Ear Hear. 2013;34(4):508-514.

15. Lambeek LC, van Tulder MW, Swinkels IC, Koppes LL, Anema JR, van Mechelen W. The trend in total cost of back pain in The Netherlands in the period 2002 to 2007. Spine (Phila Pa 1976). 2011;36(13):1050-1058.

16. Baguley D, McFerran D, Hall D. Tinnitus. Lancet. 2013;382(9904):1600-1607.

17. Langguth B. A review of tinnitus symptoms beyond 'ringing in the ears': a call to action. Curr Med Res Opin. 2011;27(8):1635-1643.

18. Pickles JO. Auditory pathways: anatomy and physiology. Handb Clin Neurol. 2015;129:3-25.

19. Axelsson A, Prasher D. Tinnitus induced by occupational and leisure noise. Noise Health. 2000;2(8):47-54.

20. Gilles A, Van Hal G, De Ridder D, Wouters K, Van de Heyning P. Epidemiology of noiseinduced tinnitus and the attitudes and beliefs towards noise and hearing protection in adolescents. PLoS One. 2013;8(7):e70297.

21. Weisz N, Hartmann T, Dohrmann K, Schlee W, Norena A. High-frequency tinnitus without hearing loss does not mean absence of deafferentation. Hear Res. 2006;222(1-2):108-114.

22. Kujawa SG, Liberman MC. Adding insult to injury: cochlear nerve degeneration after "temporary" noise-induced hearing loss. J Neurosci. 2009;29(45):14077-14085. 
23. Bauer CA, Turner JG, Caspary DM, Myers KS, Brozoski TJ. Tinnitus and inferior colliculus activity in chinchillas related to three distinct patterns of cochlear trauma. J Neurosci Res. 2008;86(11):2564-2578.

24. Norena A, Micheyl C, Chery-Croze S, Collet L. Psychoacoustic characterization of the tinnitus spectrum: implications for the underlying mechanisms of tinnitus. Audiol Neurootol. 2002;7(6):358-369.

25. De Ridder D, Elgoyhen AB, Romo R, Langguth B. Phantom percepts: tinnitus and pain as persisting aversive memory networks. Proc Natl Acad Sci U S A. 2011;108(20):8075-8080.

26. Eggermont JJ. Pathophysiology of tinnitus. Prog Brain Res. 2007;166:19-35.

27. Syka J. Plastic changes in the central auditory system after hearing loss, restoration of function, and during learning. Physiol Rev. 2002;82(3):601-636.

28. Shore SE, Roberts LE, Langguth B. Maladaptive plasticity in tinnitus - triggers, mechanisms and treatment. Nat Rev Neurol. 2016;12(3):150-160.

29. Malmierca MS. The structure and physiology of the rat auditory system: an overview. Int Rev Neurobiol. 2003;56:147-211.

30. Rammo R, Ali R, Pabaney A, Seidman M, Schwalb J. Surgical Neuromodulation of Tinnitus: A Review of Current Therapies and Future Applications. Neuromodulation. 2019;22(4):380387.

31. Roberts LE, Eggermont JJ, Caspary DM, Shore SE, Melcher JR, Kaltenbach JA. Ringing ears: the neuroscience of tinnitus. J Neurosci. 2010;30(45):14972-14979.

32. Rauschecker JP, Leaver AM, Muhlau M. Tuning out the noise: limbic-auditory interactions in tinnitus. Neuron. 2010;66(6):819-826.

33. Song JJ, Vanneste S, De Ridder D. Dysfunctional noise cancelling of the rostral anterior cingulate cortex in tinnitus patients. PLoS One. 2015;10(4):e0123538.

34. Langguth B, Kreuzer PM, Kleinjung T, De Ridder D. Tinnitus: causes and clinical management. Lancet Neurol. 2013;12(9):920-930.

35. Auerbach BD, Rodrigues PV, Salvi RJ. Central gain control in tinnitus and hyperacusis. Front Neurol. 2014;5:206.

36. Norena AJ. An integrative model of tinnitus based on a central gain controlling neural sensitivity. Neurosci Biobehav Rev. 2011;35(5):1089-1109.

37. De Ridder D, Vanneste S, Langguth B, Llinas R. Thalamocortical Dysrhythmia: A Theoretical Update in Tinnitus. Front Neurol. 2015;6:124.

38. Llinas RR, Ribary U, Jeanmonod D, Kronberg E, Mitra PP. Thalamocortical dysrhythmia: A neurological and neuropsychiatric syndrome characterized by magnetoencephalography. Proc Natl Acad Sci U S A. 1999;96(26):15222-15227.

39. Rauschecker JP, May ES, Maudoux A, Ploner M. Frontostriatal Gating of Tinnitus and Chronic Pain. Trends Cogn Sci. 2015;19(10):567-578.

40. Hoare DJ, Hall DA. Clinical guidelines and practice: a commentary on the complexity of tinnitus management. Eval Health Prof. 2011;34(4):413-420.

41. Joustra J, Buwalda J, Cima R, et al. Richtlijn Tinnitus. Nederlandse Vereniging voor Keel Neus -Oorheelkunde en Heelkunde van het Hoofd - Halsgebied. 2016.

42. Cima RFF, Mazurek B, Haider H, et al. A multidisciplinary European guideline for tinnitus: diagnostics, assessment, and treatment. HNO. 2019;67(Suppl 1):10-42.

43. Hoare DJ, Edmondson-Jones M, Sereda M, Akeroyd MA, Hall D. Amplification with hearing aids for patients with tinnitus and co-existing hearing loss. Cochrane Database of Systematic Reviews. 2014(1).

44. Hesse G. Evidence and evidence gaps in tinnitus therapy. GMS Curr Top Otorhinolaryngol Head Neck Surg. 2016;15:Doc04. 
45. Arts RA, George EL, Stokroos RJ, Vermeire K. Review: cochlear implants as a treatment of tinnitus in single-sided deafness. Curr Opin Otolaryngol Head Neck Surg. 2012;20(5):398-403.

46. Cima RF, Maes IH, Joore MA, et al. Specialised treatment based on cognitive behaviour therapy versus usual care for tinnitus: a randomised controlled trial. Lancet. 2012;379(9830):1951-1959.

47. Henry JA, Schechter MA, Zaugg TL, et al. Clinical trial to compare tinnitus masking and tinnitus retraining therapy. Acta Otolaryngol Suppl. 2006(556):64-69.

48. Phillips JS, McFerran D. Tinnitus Retraining Therapy (TRT) for tinnitus. Cochrane Database of Systematic Reviews. 2010(3).

49. Tass PA, Adamchic I, Freund HJ, von Stackelberg T, Hauptmann C. Counteracting tinnitus by acoustic coordinated reset neuromodulation. Restor Neurol Neurosci. 2012;30(2):137-159.

50. Vanneste S, van Dongen M, De Vree B, et al. Does enriched acoustic environment in humans abolish chronic tinnitus clinically and electrophysiologically? A double blind placebo controlled study. Hear Res. 2013;296:141-148.

51. Okamoto H, Stracke H, Stoll W, Pantev C. Listening to tailor-made notched music reduces tinnitus loudness and tinnitus-related auditory cortex activity. Proc Natl Acad Sci U S A. 2010;107(3): 1207-1210.

52. Sereda M, Xia J, El Refaie A, Hall DA, Hoare DJ. Sound therapy (using amplification devices and/or sound generators) for tinnitus. Cochrane Database of Systematic Reviews. 2018(12).

53. Meng Z, Liu S, Zheng Y, Phillips JS. Repetitive transcranial magnetic stimulation for tinnitus. Cochrane Database of Systematic Reviews. 2011(10).

54. Vanneste S, De Ridder D. Noninvasive and invasive neuromodulation for the treatment of tinnitus: an overview. Neuromodulation. 2012;15(4):350-360.

55. Lozano AM, Lipsman N, Bergman H, et al. Deep brain stimulation: current challenges and future directions. Nat Rev Neurol. 2019;15(3):148-160.

56. Cheung SW, Larson PS. Tinnitus modulation by deep brain stimulation in locus of caudate neurons (area LC). Neuroscience. 2010;169(4):1768-1778.

57. Cheung SW, Racine CA, Henderson-Sabes J, et al. Phase I trial of caudate deep brain stimulation for treatment-resistant tinnitus. J Neurosurg. 2019:1-10.

58. Dijkstra E, Figee M, Schuurman PR, Denys D. Effective deep brain stimulation of intractable tinnitus: A case study. Brain Stimul. 2018;11(5):1205-1207.

59. Hamani C, Temel Y. Deep brain stimulation for psychiatric disease: contributions and validity of animal models. Sci Transl Med. 2012;4(142):142rv148.

60. McIntyre CC, Hahn PJ. Network perspectives on the mechanisms of deep brain stimulation. Neurobiol Dis. 2010;38(3):329-337.

61. McConnell GC, So RQ, Hilliard JD, Lopomo P, Grill WM. Effective deep brain stimulation suppresses low-frequency network oscillations in the basal ganglia by regularizing neural firing patterns. J Neurosci. 2012;32(45):15657-15668.

62. Whitmer D, de Solages C, Hill B, Yu H, Henderson JM, Bronte-Stewart H. High frequency deep brain stimulation attenuates subthalamic and cortical rhythms in Parkinson's disease. Front Hum Neurosci. 2012;6:155.

63. Tan S, Vlamings R, Lim L, et al. Experimental deep brain stimulation in animal models. Neurosurgery. 2010;67(4):1073-1079; discussion1080. 


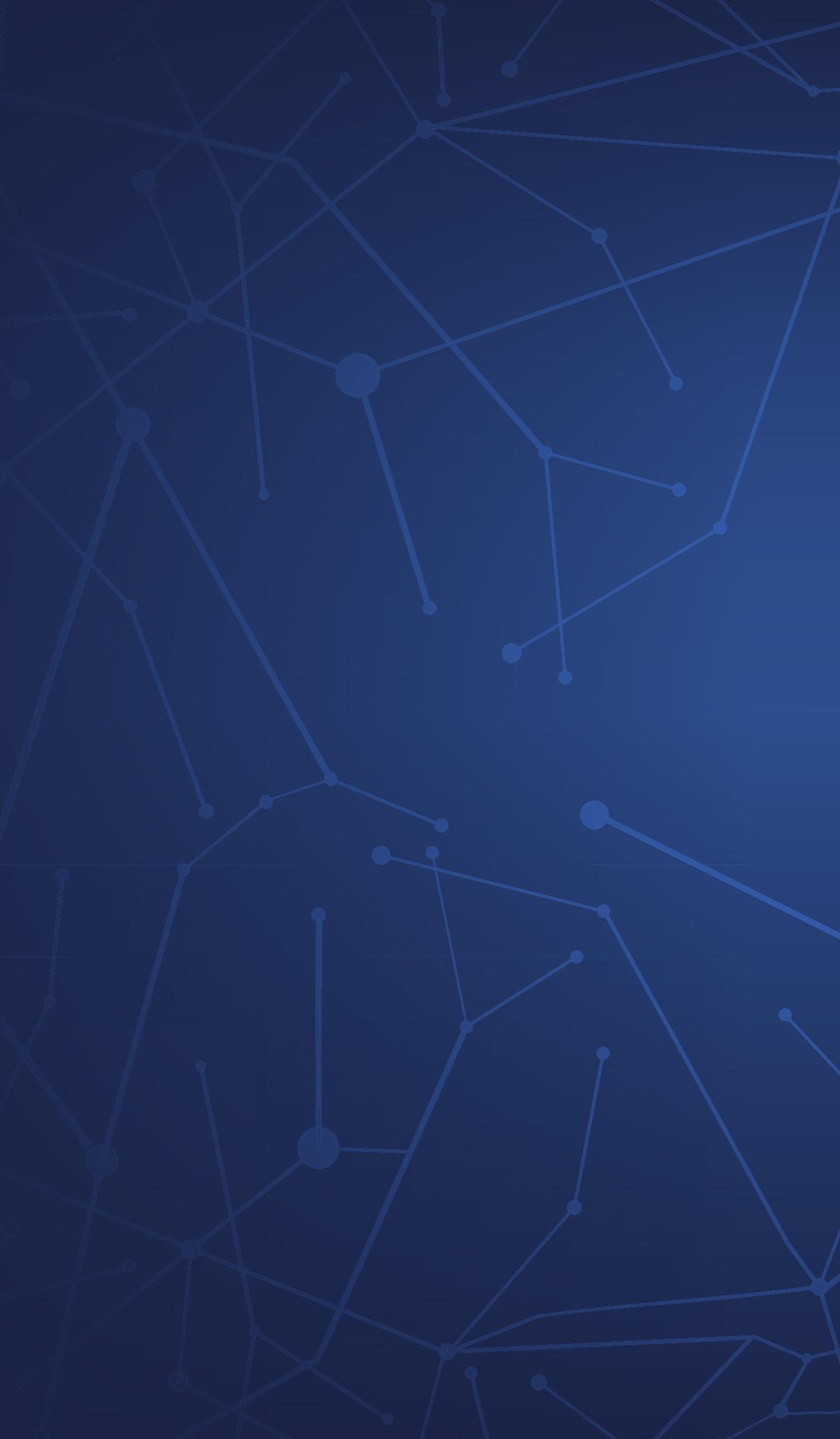




\section{PART I}

Exploring deep brain stimulation in the auditory circuit 



\title{
Tinnitus: is there a place for brain stimulation?
}

\author{
Van Zwieten G \\ Smit JV \\ Jahanshahi A \\ Temel Y \\ Stokroos RJ
}




\section{Abstract}

Tinnitus is the perception of a "phantom sound" and has a high prevalence. Although many therapies have been investigated within the last decades, there is still no effective standard therapy. Animal studies and human functional imaging studies revealed that tinnitus perception is associated with many complex changes in multiple brain structures. There is growing evidence that brain stimulation might be able to interrupt the local altered neuronal activity and hereby inhibit tinnitus perception. In this editorial review an update is given on the most promising targets for brain stimulation. Promising structures for stimulation are the dorsal cochlear nucleus, the inferior colliculus and the medial geniculate body of the thalamus. For cortical stimulation, the auditory cortex is considered as a target. Nevertheless, the field is waiting for evidence from well-designed clinical trials, based on supporting evidence from experimental/mechanistic research, to support or discourage the application of brain stimulation in tinnitus. 
Currently up to $15 \%$ of the general population suffers chronically from perception of a "phantom sound", also known as tinnitus. ${ }^{1,2}$ This is defined as the perception of a sound in the absence of an external source. Due to lack of awareness and an ageing population the prevalence is still rising. The most severe degree of tinnitus is experienced by $2.4 \%$ of the population and is associated with insomnia, depression and even suicide.,4 Although many therapies are being developed in the last years, there is still no effective standard therapy. 5,6 Current therapies mostly focus on treating the distress caused by tinnitus instead of reducing the actual phantom sound. Nevertheless, many patients do not benefit from the current approaches and become severe and chronic tinnitus sufferers. In these patients, neuromodulation-based treatments can be a promising option. Several preclinical and clinical studies demonstrated beneficial effects. ${ }^{7}$ From coincidental findings in Parkinson's disease patients who also had tinnitus and were treated with deep brain stimulation (DBS), we know that stimulation can alter or even completely diminish perception of tinnitus. ${ }^{8}$ Since central nervous system changes especially occur in the chronic patients it can be expected that these refractory, chronic and often severe sufferers are the best candidates for neuromodulation. In this editorial review, pathophysiological changes associated with tinnitus and the potential of neuromodulation to interfere with these changes are discussed. Based on the latest preclinical and clinical studies, brain stimulation of subcortical auditory targets, non-auditory structures and relevant cortical structures are reviewed. Furthermore, cochlear stimulation, as well as the novel approach trigeminal nerve stimulation to treat tinnitus are discussed.

Animal studies and human functional imaging studies revealed that tinnitus perception is associated with many complex changes in several different brain structures. The general accepted hypothesis is that neuronal changes occur in both auditory and non-auditory brain structures, most often as a compensating mechanism on reduced input from the auditory nerve caused by cochlear hair cell damage, which is associated with hearing loss. These central neuronal changes include an increase in spontaneous firing rate, synchronized activity, bursting activity and tonotopic reorganization.9,10 Tinnitus perception is the result of dysfunction of multiple involved brain structures. The exact working mechanism of DBS is unknown, but different theories describe a combined excitatory and inhibitory effect.11-14 DBS has been shown to be able to reduce an increased spontaneous activity as this therapy inhibits the elevated bursting 
activity in the subthalamic nucleus in Parkinson's disease patients. ${ }^{15}$ It can be expected that modulation of one arbitrary part in the complex tinnitus pathways can disrupt pathological neuronal activity and thereby alter tinnitus perception or distress caused by this phantom sensation.7,16

Complex interactions within and between auditory and non-auditory brain structures are present in tinnitus. Every change in neuronal activity causes a cascade of changes in direct and indirect connected brain areas. An important role for the limbic system has been implied, as studies have shown that attention and emotions can influence tinnitus perception. ${ }^{4,17}$ To simplify the complex pathways, classical and non-classical auditory pathways are distinguished, not taking descending projections into account. Following the classical pathway, the cochlear nerve fibers end in the ipsilateral cochlear nucleus $(\mathrm{CN})$, further project to the central part of the mainly contralateral inferior colliculus (IC) and subsequently to the medial geniculate body (MGB) and primary auditory cortex. Brain structures in the non-classical auditory pathway are interestingly less tonotopically organized than in the classical pathway and have additional connections with the limbic system and caudate nucleus. The central part of the IC connects to the dorsal and external nucleus of the IC, which in turn projects to the dorsal and medial part of the MGB. From these parts of the MGB, connections project to the amygdala, secondary auditory cortex and association auditory cortex. ${ }^{2,18}$

The loudness of tinnitus does not always correlate with the burden and impact of tinnitus on life quality, suggesting a substantial role of non-auditory brain structures in the pathophysiology of chronic tinnitus. The importance of auditory-limbic interactions has been emphasized by Rauschecker et al., who propose a failing neural "noise cancellation" mechanism of (para)limbic structures as the underlying cause of tinnitus suffering. ${ }^{19}$

Multiple targets for DBS can be proposed within the auditory pathway, which might have an advantageous effect on tinnitus perception (Figure 2.1). Firstly, multiple preclinical and clinical studies suggest that the dorsal cochlear nucleus (DCN) plays an important role in the development of tinnitus and could therefore be a target for DBS in tinnitus. An increased bursting activity is found in both the DCN and ventral cochlear nucleus (VCN). ${ }^{20,21}$ Ablation of this structure in an animal study resulted in a decrease in neuronal hyperactivity in higher output structures.22 In a human study, patients who did not have a 
functioning auditory nerve received an auditory brainstem implant in the DCN. The majority of successfully implanted patients (6/10) reported reduction in tinnitus perception or even complete suppression (1/10) during stimulation..$^{23}$ Side-effects of stimulation that have been described include facial pain and ocular vibration, although some studies do not mention any sideeffects. ${ }^{23,24}$ Effects of stimulation on hearing in patients with intact auditory nerves are not known. Secondly, IC stimulation might have an effect on tinnitus perception since studies have shown an increased spontaneous activity and neuronal synchrony in the contralateral IC in tinnitus. ${ }^{25-28}$ Almost all ascending auditory brainstem projections converge in the IC. Electrical stimulation of the IC in patients with unilateral deafness showed some sideeffects, including perception of unpleasant sounds, paraesthesia, dizziness, facial twitches and temperature changes. ${ }^{29}$ Thirdly, the MGB is a possible target in the auditory pathway. It is known that the thalamus plays an important role in tinnitus, as thalamotomies have shown attenuation of tinnitus. Integration of auditory and limbic information occurs in the thalamus and, more specifically, the amygdala receives auditory input from the MGB. ${ }^{17}$ The MGB has an important role in tinnitus, since the ventromedial prefrontal cortex and nucleus accumbens (NAc) might be able to tune out the pathophysiological tinnitus signal by projecting to the MGB. ${ }^{19,30}$ Although the role of the MGB in tinnitus is less intensively investigated as compared to the DCN and IC, it can be expected that stimulation of this specific thalamic structure can influence tinnitus perception and distress. Side-effects of electrical stimulation of the MGB are not known. Thalamic stimulation for movement disorders, however, has been proven to be safe with only few reversible side-effects. ${ }^{31}$ The MGB is better accessible with stereotaxy than deeper auditory structures and therefore the risks of surgery are expected to be relatively low.

Coincidental findings in patients with movement disorders who were treated with DBS taught us that stimulation of non-auditory targets can attenuate tinnitus. Stimulation of the ventral intermediate nucleus of the thalamus (VIM) in Parkinson's disease patients who also suffered from tinnitus improved tinnitus in three out of seven patients. ${ }^{8}$ Furthermore, two case reports described a decrease in tinnitus perception after a cerebrovascular accident in the putamen and caudate nucleus and after perioperative focal vascular injury in area LC, a locus of the caudate nucleus. ${ }^{32,33}$ A clinical study where patients with movement disorders were temporarily stimulated in locus LC revealed a decrease in tinnitus loudness in all patients. ${ }^{34}$ Although we do not know much 
about the role of the VIM or caudate nucleus in the pathophysiology in tinnitus, abovementioned findings are encouraging. The NAc, also known as the "reward centre" of the brain, has a role in tinnitus distress according to clinical electroencephalographic findings. ${ }^{35}$ It is hypothesized that DBS of the NAc in tinnitus would disrupt the abnormal functioning NAc in tinnitus patients in a way that tinnitus perception would be inhibited $1{ }^{19}$ DBS of the NAc has been performed in obsessive compulsive disorder patients and is associated with a risk of hypomania. ${ }^{36}$ Human functional magnetic resonance imaging studies and preclinical studies have demonstrated involvement of the amygdala and hippocampus in tinnitus and these areas could therefore be considered as possible DBS targets. ${ }^{37}$ Side-effects like negative emotions have appeared in some patients during stimulation and make these areas are less suitable for the treatment of tinnitus with DBS.

Other neuromodulation-based approaches have also been suggested. In this respect, modulating the activity of relevant cortical structures has been performed. Transcranial Magnetic Stimulation (TMS) is a non-invasive technique in which strong magnetic field impulses can alter neuronal activity in cortical but also in areas connected to the cortex. Repetitive TMS can induce residual inhibition and suppress tinnitus loudness temporarily. ${ }^{7}$ The effect of another non-invasive therapy, transcranial direct current stimulation (tDCS), has been evaluated in a meta-analysis. ${ }^{38}$ Overall, $39.5 \%$ of the patients responded with an average decrease in tinnitus intensity of $13.5 \%$. This effect can last for an hour or longer. Another method of modulating the cortical activity is by extradural electrical stimulation. Stimulation of the primary auditory cortex and/or the secondary auditory cortex can be successful in suppressing severe, refractory tinnitus. ${ }^{39}$ De Ridder and colleagues ${ }^{40}$ implanted auditory cortex electrodes in 43 tinnitus patients who all showed benefit from two placebo controlled TMS sessions. In this technique, the electrodes are secured on the dura of the auditory cortex, which is reached via a craniotomy $(2 \times 6 \mathrm{~cm})$, guided by functional magnetic resonance imaging. Despite that all patients responded to TMS, only $67 \%$ responded to cortical stimulation with an average suppressing effect of $51 \%$. Side-effects of stimulation are limited and only occurred at high frequency or high intensity stimulation. Symptoms as a feeling of intoxication, word finding difficulties, dizziness, vertigo, hearing perception changes, feeling of "aural pressure" and out of body experiences were described. Complications can be severe. Epileptic seizures occurred in three of forty-three patients and of the four patients who were implanted 
following the intradural technique, one had an intracranial haemorrhage and one developed an intracranial abscess. In another recent study, chronic electrical stimulation of the auditory cortex was applied in 9 patients. The authors did not find a general objective efficiency. ${ }^{41}$ Overall, cortical electrical stimulation might become a beneficial treatment option for a subgroup of severe tinnitus patients. ${ }^{42,43}$

Besides DBS and cortical neuromodulation approaches, some other concepts have been described. Intracochlear stimulation via cochlear implantation is a viable treatment option in patients with tinnitus and unilateral of bilateral severe or profound hearing loss. ${ }^{44,45}$ In patients with bilateral hearing loss, a systematic review concluded a reduction of mean tinnitus score of $25-72 \%$ and a total suppression of tinnitus in $8-45 \%$ of patients. ${ }^{46}$ Standard clinical stimulation, stimulation independent of an acoustic input and even inaudible stimulation can be effective. This suggests an effect of central neuroplastic changes besides the effect of a shift in attention from tinnitus to environmental sounds. ${ }^{47}$ Another technique that can indirectly influence central tinnitus related neuronal activity is non-invasive Transcutaneous Electric Nerve Stimulation (TENS). ${ }^{39}$ The cochlear nuclei receive somatosensory, non-auditory inputs besides auditory inputs from the vestibulocochlear nerve. Preclinical studies showed that transcutaneous electrical stimulation of the branches of the trigeminal nerve and parts of the dorsal column cause modulation of neuronal activity in the DCN. ${ }^{48}$ TENS of the median nerve, temporomandibular joint, parts of the external ear and upper cervical nerve C2 can be used to inhibit tinnitus perception temporarily in some patients. ${ }^{49-52}$ Recently, electrical stimulation of branches of the trigeminal nerve or the trigeminal ganglion has been proposed as a potential treatment modality for tinnitus. $39,53,54$

In conclusion, developments in the field of neuromodulation are promising for patients with severe tinnitus. Several types of neuromodulation-based approaches are being investigated. The general mechanism of action is that neuromodulation interferes with pathological neuronal activity and thereby can attenuate distress or perception of tinnitus. In this respect, increased neuronal activity is found in the DCN, IC, MGB and auditory cortex. These regions are therefore potential targets for brain stimulation. It is impossible to reach these regions selectively and precisely with non-invasive stimulation methods. When surgery is considered, then the MGB is a more accessible target. Furthermore, the MGB is an important relay station where the auditory 
and limbic structures interact. Tinnitus perception can be influenced with superficial stimulation techniques, which attenuate abnormal auditory cortex activity. Up to date, only a subgroup of tinnitus patients responded to auditory cortex stimulation. From the non-auditory structures, stimulation of the VIM, caudate nucleus (locus LC) and NAc have potential to interfere with tinnitus. Using a bottom-up approach with cochlear stimulation or TENS of somatosensory inputs of the DCN, tinnitus percept can be modified in some cases.

Although much is happening at the moment, the field is waiting for evidence from well-designed clinical trials, based on supporting evidence from experimental and mechanistic research, to support or discourage the application of brain stimulation in tinnitus.

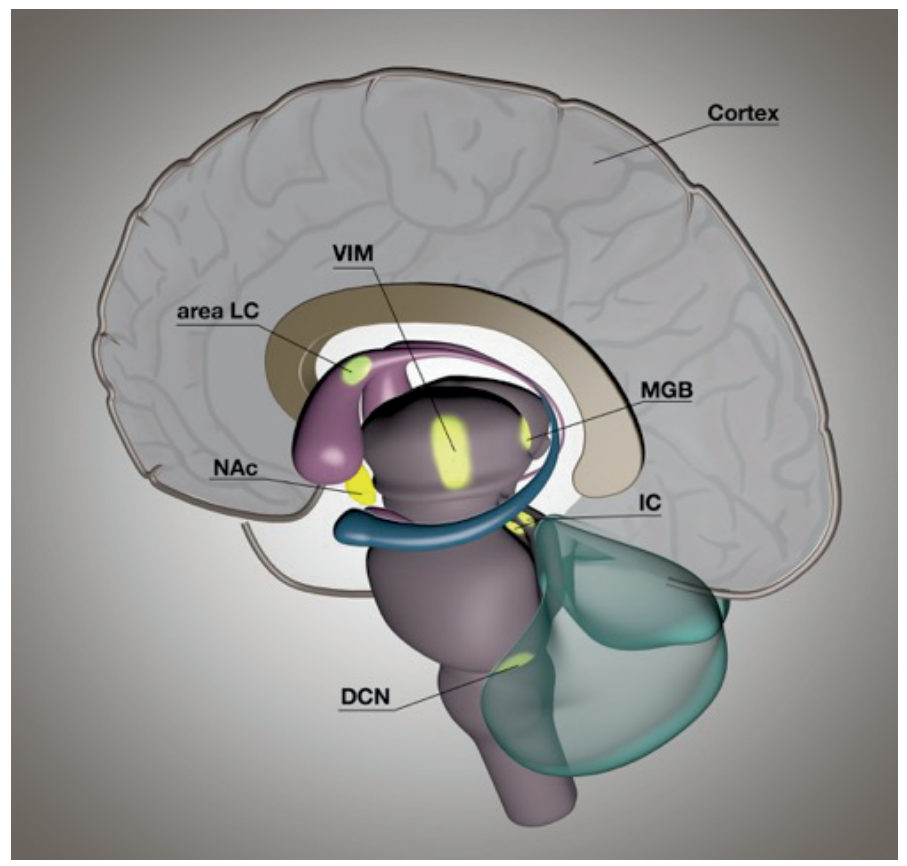

Figure 2.1 A schematic representation of a human brain from a sagittal view, showing possible targets for brain stimulation to treat tinnitus. Auditory structures include the dorsal cochlear nucleus (DCN), inferior colliculus (IC), medial geniculate body of the thalamus (MGB) and auditory cortex. Non-auditory structures are the nucleus accumbens (NAc), locus of caudate neurons (area LC) and ventral intermediate nucleus of the thalamus (VIM). 


\section{References}

1. Heller AJ. Classification and epidemiology of tinnitus. Otolaryngol Clin North Am. 2003; 36(2):239-248.

2. Møller A. Epidemiology of Tinnitus in Adults, in: Textbook of Tinnitus. 2011.

3. Axelsson A, Ringdahl A. Tinnitus--a study of its prevalence and characteristics. Br J Audiol. 1989;23(1):53-62.

4. Langguth B, et al. Tinnitus and depression. World J Biol Psychiatry. 2011;12(7):489-500.

5. Hoare DJ, et al. Systematic review and meta-analyses of randomized controlled trials examining tinnitus management. Laryngoscope. 2011;121(7):1555-1564.

6. Landgrebe $\mathrm{M}$, et al. Methodological aspects of clinical trials in tinnitus: a proposal for an international standard. J Psychosom Res. 2012;73(2):112-121.

7. Vanneste S, De Ridder D. Noninvasive and invasive neuromodulation for the treatment of tinnitus: an overview. Neuromodulation. 2012;15(4):350-360.

8. Shi Y, et al. Deep brain stimulation effects in patients with tinnitus. Otolaryngol Head Neck Surg. 2009;141(2):285-287.

9. Eggermont JJ, Roberts LE. The neuroscience of tinnitus. Trends Neurosci. 2004;27(11):676-682.

10. Salvi RJ, Wang J, Ding D. Auditory plasticity and hyperactivity following cochlear damage. Hear Res. 2000;147(1-2):261-274.

11. Kringelbach ML, et al. Translational principles of deep brain stimulation. Nat Rev Neurosci. 2007;8(8):623-635.

12. Chiken S, Nambu A. Mechanism of Deep Brain Stimulation: Inhibition, Excitation, or Disruption? Neuroscientist. 2016;22(3):313-322

13. Benazzouz A, Hallett M. Mechanism of action of deep brain stimulation. Neurology. 2000; 55(12 Suppl 6):S13-S16.

14. McIntyre CC, et al. Uncovering the mechanism(s) of action of deep brain stimulation: activation, inhibition, or both. Clin Neurophysiol. 2004;115(6):1239-1248.

15. Benazzouz A, et al. Intraoperative microrecordings of the subthalamic nucleus in Parkinson's disease. Mov Disord. 2002;17 Suppl 3:S145-S149.

16. Smit JV, et al. Deep brain stimulation in tinnitus: current and future perspectives. Brain Res. 2015;1608:51-65.

17. Kraus KS, Canlon B. Neuronal connectivity and interactions between the auditory and limbic systems. Effects of noise and tinnitus. Hear Res. 2012;288(1-2):34-46.

18. Kandel ER, Schwartz JH, Jessell TM, Principles of Neural Science, ed. H.P.D. McGraw-Hill. 2000.

19. Rauschecker JP, Leaver AM, Muhlau M. Tuning out the noise: limbic-auditory interactions in tinnitus. Neuron. 2010;66(6):819-826.

20. Vogler DP, Robertson D, Mulders WH. Hyperactivity in the ventral cochlear nucleus after cochlear trauma. J Neurosci. 2011;31(18):6639-6645.

21. Kaltenbach JA. Summary of evidence pointing to a role of the dorsal cochlear nucleus in the etiology of tinnitus. Acta Otolaryngol Suppl. 2006;(556):20-26.

22. Manzoor NF, et al. Noise-induced hyperactivity in the inferior colliculus: its relationship with hyperactivity in the dorsal cochlear nucleus. J Neurophysiol. 2012;108(4):976-988.

23. Soussi T, Otto SR. Effects of electrical brainstem stimulation on tinnitus. Acta Otolaryngol. 1994;114(2):135-140.

24. Matthies $\mathrm{C}$, et al. Auditory brainstem implants: current neurosurgical experiences and perspective. J Laryngol Otol Suppl. 2000;(27):32-36. 
25. Bauer CA, et al. Tinnitus and inferior colliculus activity in chinchillas related to three distinct patterns of cochlear trauma. J Neurosci Res. 2008;86(11):2564-2578.

26. Chen GD, Jastreboff PJ. Salicylate-induced abnormal activity in the inferior colliculus of rats. Hear Res. 1995;82(2):158-178.

27. Brozoski TJ, Ciobanu L, Bauer CA. Central neural activity in rats with tinnitus evaluated with manganese-enhanced magnetic resonance imaging (MEMRI). Hear Res. 2007;228(1-2):168-179.

28. Lanting $\mathrm{CP}$, et al. Functional imaging of unilateral tinnitus using fMRI. Acta Otolaryngol. 2008;128(4):415-421.

29. Lim HH, et al. The auditory midbrain implant: effects of electrode location. Hear Res. 2008; 242(1-2):74-85.

30. Leaver AM, et al. Dysregulation of limbic and auditory networks in tinnitus. Neuron. 2011; 69(1):33-43.

31. Koller WC, et al. Long-term safety and efficacy of unilateral deep brain stimulation of the thalamus in essential tremor. Mov Disord. 2001;16(3):464-468.

32. Larson PS, Cheung SW. A stroke of silence: tinnitus suppression following placement of a deep brain stimulation electrode with infarction in area LC. J Neurosurg. 2013;118(1):192-194.

33. Lowry LD, Eisenman LM, Saunders JC. An absence of tinnitus. Otol Neurotol. 2004;25(4): 474-478.

34. Cheung SW, Larson PS. Tinnitus modulation by deep brain stimulation in locus of caudate neurons (area LC). Neuroscience. 2010;169(4):1768-1778.

35. Vanneste S, et al. The neural correlates of tinnitus-related distress. Neuroimage. 2010;52(2): 470-480.

36. de Koning PP, et al. Current status of deep brain stimulation for obsessive-compulsive disorder: a clinical review of different targets. Curr Psychiatry Rep. 2011;13(4):274-282.

37. Shulman A, et al. SPECT Imaging of Brain and Tinnitus-Neurotologic/Neurologic Implications. Int Tinnitus J. 1995;1(1):13-29.

38. Song JJ, et al. Transcranial direct current stimulation in tinnitus patients: a systemic review and meta-analysis. ScientificWorldJournal. 2012;2012:427941.

39. Soleymani $\mathrm{T}$, et al. Surgical approaches to tinnitus treatment: A review and novel approaches. Surg Neurol Int. 2011;2:154.

40. De Ridder D, et al. Transcranial magnetic stimulation and extradural electrodes implanted on secondary auditory cortex for tinnitus suppression. J Neurosurg. 2011;114(4):903-911.

41. Engelhardt J, et al. Effect of chronic cortical stimulation on chronic severe tinnitus: a prospective randomized double-blind cross-over trial and long-term follow up. Brain Stimul. 2014;7(5):694-700.

42. De Ridder D, Vanneste S. Auditory cortex stimulation might be efficacious in a subgroup of tinnitus patients. Brain Stimul. 2014;7(6):917-918.

43. Langguth B, De Ridder D. Tinnitus: therapeutic use of superficial brain stimulation. Handb Clin Neurol. 2013;116:441-467.

44. Arts RA, et al. Review: cochlear implants as a treatment of tinnitus in single-sided deafness. Curr Opin Otolaryngol Head Neck Surg. 2012;20(5):398-403.

45. Tyler RS, et al. Electrical Stimulation of the Cochlea to Reduce Tinnitus. Semin Hear. 2008; 29(4):326-332.

46. Ramakers GG, et al. The effect of cochlear implantation on tinnitus in patients with bilateral hearing loss: A systematic review. Laryngoscope. 2015;125(11):2584-2592.

47. Arts RA, et al. Tinnitus Suppression by Intracochlear Electrical Stimulation in Single-Sided Deafness: A Prospective Clinical Trial - Part I. Audiol Neurootol. 2015;20(5):294-313. 
48. Wu C, Martel DT, Shore SE. Transcutaneous induction of stimulus-timing-dependent plasticity in dorsal cochlear nucleus. Front Syst Neurosci. 2015;9:116.

49. Moller AR, Moller MB, Yokota M. Some forms of tinnitus may involve the extralemniscal auditory pathway. Laryngoscope. 1992;102(10):1165-1171.

50. Herraiz C, Toledano A, Diges I. Trans-electrical nerve stimulation (TENS) for somatic tinnitus. Prog Brain Res. 2007;166:389-394.

51. Steenerson RL, Cronin GW. Tinnitus reduction using transcutaneous electrical stimulation. Otolaryngol Clin North Am. 2003;36(2):337-344.

52. Vanneste $S$, et al. Transcutaneous electrical nerve stimulation (TENS) of upper cervical nerve (C2) for the treatment of somatic tinnitus. Exp Brain Res. 2010;204(2):283-287.

53. Dehmel S, Cui YL, Shore SE. Cross-modal interactions of auditory and somatic inputs in the brainstem and midbrain and their imbalance in tinnitus and deafness. Am J Audiol. 2008; 17(2):S193-209.

54. Shore S, Zhou J, Koehler S. Neural mechanisms underlying somatic tinnitus. Prog Brain Res. 2007;166:107-123. 



\section{Alleviation of tinnitus with high frequency stimulation of the dorsal cochlear nucleus: a rodent study}

Van Zwieten G

Jahanshahi A

Van Erp ML

Temel $Y$

Stokroos RJ

Janssen MLF

Smit JV

Published in Trends in Hearing,

January 2019, Volume 22. 


\section{Abstract}

Deep brain stimulation of the central auditory pathway is emerging as a promising treatment modality for tinnitus. Within this pathway the dorsal cochlear nucleus (DCN) plays a key role in the pathophysiology of tinnitus and is believed to be a "tinnitus generator". We hypothesized that high frequency stimulation (HFS) of the DCN would influence tinnitus-related abnormal neuronal activity within the auditory pathway and hereby suppress tinnitus. To this end, we assessed the effect of HFS of the DCN in a noise-induced rat model of tinnitus. Presence of tinnitus was verified using the gap-prepulse inhibition of the acoustic startle response paradigm. Hearing thresholds were determined before and after noise trauma by measuring the auditory brainstem responses. Additionally, changes in neuronal activity induced by noise trauma and HFS were assessed using c-Fos immunohistochemistry in related structures. Results showed tinnitus development after noise-trauma and hearing loss ipsilateral to the side exposed to noise trauma. During HFS of the DCN, tinnitus was suppressed. There was no change in c-Fos expression within the central auditory pathway after HFS. These findings suggest that DCN-HFS changes patterns of activity and results in information lesioning within the network and hereby blocking the relay of abnormal tinnitus related neuronal activity. 


\section{Introduction}

Subjective tinnitus is defined as an auditory perception that is not induced by an acoustic stimulus. This symptom is highly prevalent in the general population and can severely impair an individual's quality of life. In recent years, numerous studies have provided further insight into tinnitus-related changes in auditory and non-auditory brain structures that occur, which are usually triggered by hearing loss. ${ }^{1-4}$

The dorsal cochlear nucleus (DCN) is the first site of multisensory convergence in the auditory pathway. Previous studies have suggested an important role of the DCN in the pathophysiology of tinnitus. ${ }^{5-7}$ It has been shown that a functioning DCN is necessary for tinnitus to develop in animal models. ${ }^{5,8}$ Therefore, the DCN has been referred as a tinnitus trigger and generator. ${ }^{9}$ Following this theory, hearing loss leads to reduced input to the eighth cranial nerve, and as a compensatory mechanism the DCN shows hyperactivity, e.g. increased synchrony and bursting. ${ }^{7}$ Multiple synaptic changes underlying these hyperactive changes have been described, such as degeneration of fibers, changes in excitatory neurotransmission, alterations in the balance of excitatory and inhibitory synapses and changes in expression of glycine receptors. ${ }^{10}$ Increased neuronal synchrony and bursting activity are also described in upstream areas of the auditory pathway, in specific the inferior colliculus (IC), ${ }^{11-13}$ medial geniculate body of the thalamus (MGB) ${ }^{14}$ and primary auditory cortex (A1). ${ }^{15,16}$ These findings suggest that a neural code for tinnitus emerges in the DCN, but affects the full network.

Despite decades of efforts to develop an effective therapy, it is still highly challenging to treat tinnitus. Multiple preclinical and clinical studies suggested deep brain stimulation (DBS) as a promising treatment option in severe, refractory tinnitus. ${ }^{17-19}$ Furthermore, electrical stimulation of the DCN with an auditory brainstem implant (ABI), which is used to elicit auditory sensations, can lead to changes in tinnitus loudness. ${ }^{20}$ This has been studied in patients with neurofibromatosis type 2 who received an ABI to elicit auditory sensations. DBS is a less invasive modality to electrically stimulate a subcortical structure, yet implantation of the DCN with a DBS electrode has not been performed in a clinical setting. 
Although the exact mechanism behind the effects of DBS remains unclear, there is evidence that especially high-frequency stimulation (HFS) disrupts abnormal neuronal activity. ${ }^{21,22}$ It has been proposed that a high rate conditioner stimulus induces stochastic characteristics of resting-level spontaneous activity, resulting in tinnitus suppression.23,24 In Parkinson's disease, hypersynchrony and bursting activity can be suppressed with HFS of the subthalamic nucleus. ${ }^{21,25}$ Therefore, we hypothesize that HFS of the DCN would suppress tinnitus with a similar mechanism.

In this study, the effect of bilateral HFS of the DCN on tinnitus was assessed in a noise induced rat model of tinnitus. Auditory brainstem responses (ABR) were recorded before and after noise exposure. Gap-prepulse inhibition of the acoustic startle (GPIAS) response paradigm was used to assess tinnitus. To elaborate on the effects of HFS as well as noise trauma on neuronal activity, the expression of an immediate early gene (c-Fos) was measured in the IC, MGB and A1.

\section{Materials and methods}

\section{Subjects}

Ten male Sprague Dawley Rats were used in the study, weighing approximately 250-300 $\mathrm{g}$ at time of surgery. Rats were individually housed in standard Makrolon ${ }^{\mathrm{TM}}$ cages, with feed and water ad libitum. Conditions in the room were constant, with a temperature of $20-22^{\circ} \mathrm{C}$ and a humidity of $60-70 \%$. The light-dark cycle was reversed and experiments were conducted within the dark period. The study protocol was approved by the Animal Experiments and Ethics Committee of Maastricht University.

\section{Study design}

A repeated measures design was used. All subjects were tested in a healthy condition and after noise trauma. All rats underwent surgery at the beginning of the experiment (Figure 3.1). 


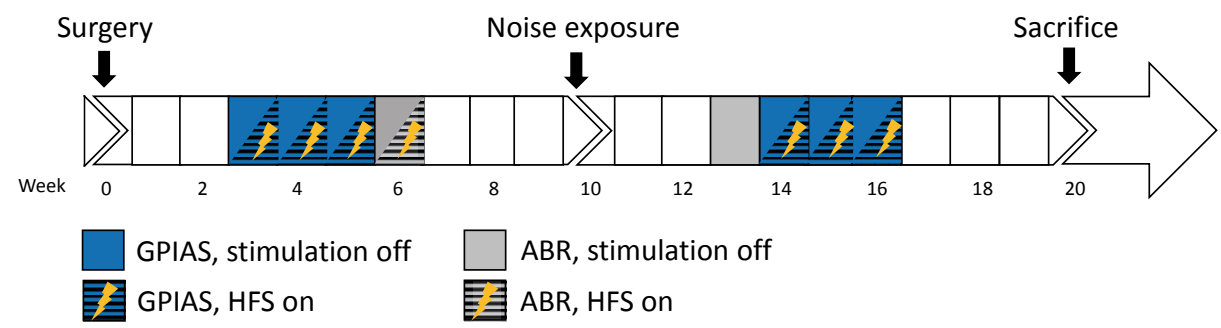

Figure 3.1 Timeline of the experimental procedures. GPIAS $=$ Gap-Prepulse Inhibition of the Acoustic Startle reflex paradigm for tinnitus assessment, ABR $=$ Auditory Brainstem Response recordings, HFS = high-frequency stimulation.

\section{Surgical procedure}

Anesthesia was induced by an intraperitoneal injection of Xylazine $(10 \mathrm{mg} / \mathrm{kg})$ and Ketamine $(90 \mathrm{mg} / \mathrm{kg})$ and maintained with Ketamine $(60 \mathrm{mg} / \mathrm{kg} / \mathrm{h})$. A rodent stereotact (Stoelting, Wood Dale, IL, USA, model 51653) was used with blunt ear bars to prevent damage to the middle ears. Stimulation electrodes (coaxial gold-plated with a platinum-iridium inner wire, shaft diameter of $250 \mu \mathrm{m}$ and tip diameter of $50 \mu \mathrm{m}$ (Technomed, Beek, the Netherlands)) were bilaterally implanted in the DCN (coordinates from Bregma: AP -11.1, ML -3.9, DV-7.8).26,27 Additionally, two recording electrodes (Teflon-coated stainlesssteel wire electrodes with exposed tip) were permanently secured to the scalp of the rats, one on the vertex and one behind the mastoid bone, to record ABR. The electrode construct was secured with five stainless steel screws and dental acrylic (Paladur, Heraeus Kulzer GmbH, Hanau Germany).

\section{Deep brain stimulation}

Rats were tested in two conditions: stimulation off (attached to the stimulation cable) and during HFS (100 Hz, $60 \mu$ s pulse width and $100 \mu \mathrm{A}$ amplitude). Electrical stimulation was bipolar with monophasic pulses, applied with a stimulator (DS8000, WPI, Berlin, Germany) connected to a constant-current isolator (DLS100, WPI, Berlin, Germany). The chosen stimulation parameters were based on our previous experiments. ${ }^{17,18,26}$ For GPIAS and ABR measurements, electrical stimulation was applied continuously during the full acquisition time, for GPIAS this included 10 minutes of habituation. 


\section{Tinnitus induction}

Subjects were anesthetized (induction with intraperitoneal injection of Xylazine $(10 \mathrm{mg} / \mathrm{kg})$ and Ketamine $(90 \mathrm{mg} / \mathrm{kg})$ and maintained with Ketamine $(60 \mathrm{mg} / \mathrm{kg} / \mathrm{h}))$ and unilaterally exposed to a $16 \mathrm{kHz}$ octave-band noise at $115 \mathrm{~dB}$ for 90 minutes. The sound paradigm was designed by a custom-made Matlab script, processed with an external soundcard with a sampling rate of $192 \mathrm{kHz}$ (Creative E-MU 0204) and amplified (Ultrasonic power amplifier and Ultrasonic Dynamic Speaker Vifa [Avisoft Bioacoustics, Berlin, Germany]). A loudspeaker was placed at a standard distance of $3 \mathrm{~mm}$ to the right ear, while the left ear was protected with a plug of modeling clay. Complete removal of the plug after the experiment could be easily achieved and confirmed with visual inspection. After noise exposure, subjects were not tested for two weeks.

\section{Gap-Prepulse Inhibition of the Acoustic Startle (GPIAS)}

To assess presence of tinnitus, GPIAS testing was performed as previously described. ${ }^{17,19}$ There were four conditions 1) at baseline, stimulation off, 2) at baseline with HFS, 3) after noise-exposure, stimulation off, and 4) after noise exposure, with HFS. The rats were placed in a cylinder made of vertical aluminum bars and polyethylene floor (diameter $17 \mathrm{~cm}$, height $40 \mathrm{~cm}$ ), inside an acoustic chamber. The stimulation electrode with swivel was attached to the electrode construct. Startle force was measured with a piezo transducer (FSG15N1A, Honeywell, Canada) underneath the cylinder floor. In the ceiling of the testing chamber, a speaker was mounted (Ultrasonic Dynamic Speaker Vifa (Avisoft Bioacoustics, Berlin, Germany). Sounds were amplified (Ultrasonic power amplifier Avisoft Bioacoustics, Berlin, Germany) and calibrated (Bruel \& Kjaer 2231 decibel meter with a 4191 microphone).

Background signals consisted of broadband noise (BBN) or narrow-band noise of $10,12,16$ or $20 \mathrm{kHz}$ at $75 \mathrm{~dB}$. The startle stimulus was a $20 \mathrm{~ms}$ long $115 \mathrm{~dB}$ peak equivalent sound pressure level (peSPL) broadband noise burst. In gap trials, there was a silent gap of $50 \mathrm{~ms}$, prior $(100 \mathrm{~ms})$ to the startle stimulus. For each of the different background signals, 10 gap-trials and 10 startle-only trials were presented with a random variable stimulus interval of $20 \pm 5 \mathrm{~s}$. The gap:no-gap ratio was calculated by dividing the amplitude of each gap startle by the corresponding mean of no-gap startles. For each condition, two complete sessions were performed on separate days and the mean of these gap:no-gap ratio was used for further analysis. Responses that contained too disturbing (moving) artifacts were excluded from analysis. Prior to every 
session, subjects were acclimatized for 5 minutes in the startle chamber, followed by 10 startle-trials in order to habituate the startle response. Furthermore, one complete session was performed at the start of the experiment for habituation to the testing procedure.

\section{Auditory Brainstem Responses (ABR)}

Hearing thresholds were assessed before noise-trauma and two weeks after noise trauma using ABR measurements. Furthermore, hearing thresholds during HFS were assessed before noise trauma. The exact procedure is described elsewhere. ${ }^{28}$ Briefly, subjects were anesthetized (see protocol above) and placed in a sound-attenuating Faraday cage. Cables were connected to the sockets of the recording electrodes in the construct on the animal's head and a ground electrode was placed on the left front paw. 1000 -ms tone bursts of 10, $12,16,20,24$ and $32 \mathrm{kHz}$ and a $\cos ^{2}$ rise and fall filter were created with Matlab and presented unilaterally with a frequency of $50 \mathrm{~Hz}$ at decreasing intensities from 110 to $0 \mathrm{~dB}$ peSPL with steps of $10 \mathrm{~dB}$. The contralateral ear was plugged with clay. Auditory stimuli were calibrated (Bruel \& Kjaer 2231 decibel meter with a 4191 microphone) and digitally triggered. ABRs were recorded in LabChart Pro 7 (ADInstruments, Castle Hill, Australia) and raw data were imported into Matlab. The evoked responses were amplified 100000 times, band-pass filtered (300-3000 Hz) and averaged. Data containing DBS artifacts were automatically removed based on a peak-detection analysis using a manual depicted maximal baseline value. The auditory threshold was defined as the lowest decibel level (peSPL) of the stimuli that produced a distinctive $\mathrm{ABR}$, in which at least two peaks (positive or negative) had to be clearly visible (Figure 3.4b).27

\section{Tissue collection}

For post mortem analysis, one noise-exposed animal who completed the whole protocol but received sham stimulation was added. Subjects were divided into sham $(n=5)$ and HFS groups $(n=6)$. Two hours prior to transcardial perfusion, the HFS group received HFS for 60 minutes, while the sham rats were only connected to the stimulation cable without stimulation. This was followed by 60 minutes of rest in their normal cages. Afterwards, rats were anesthetized with an overdose pentobarbital and perfusion-fixation was performed with Tyrode solution ( $0.1 \mathrm{M})$ followed by fixative containing $4 \%$ paraformaldehyde, $15 \%$ picric acid, and $0.05 \%$ glutaraldehyde in $0.1 \mathrm{M}$ phosphate buffer ( $\mathrm{pH} 7.6)$. 
Brains were collected and post-fixed overnight in paraformaldehyde at $4^{\circ} \mathrm{C}$ and subsequently in $1 \% \mathrm{NaN}_{3}$ at $4^{\circ} \mathrm{C}$ for long-term storage. Brains were cut serially on a vibratome (Leica ${ }^{\circledR}$, Wetzlar, Germany) into $30 \mu \mathrm{m}$ thick coronal sections while embedded in 10\% gelatin (Sigma-Aldrich, Zwijndrecht, the Netherlands).

\section{Histological and immunohistochemical staining}

For electrode verification, sections containing the electrode trajectories were processed for a cresyl violet (Nissl) staining. For c-Fos immunohistochemistry, sections were incubated for two nights with polyclonal rabbit anti-c-Fos primary antibody (1:500; Santa Cruz Biotechnology Inc, Santa Cruz, USA) followed by biotinylated donkey anti-rabbit secondary antibody (1:400; Jackson Immunoresearch Laboratories Inc., Westgrove, USA) and avidin-biotin peroxidase complex (1:800; Elite ABC-kit, Vectastain ${ }^{\circledR}$, Burlingame, CA, USA). The staining was visualized by 3,3'-Diaminobenzidine (DAB) combined with $\mathrm{NiCl} 2$ intensification.

\section{Quantitative cell counting}

The immunohistochemically stained sections were used to evaluate total number of c-Fos positive cells within the MGB, IC and A1. Stereological quantification was carried out with a stereological computer microscopy system (Stereo Investigator, Microbrightfield Bioscience, Williston, VT, USA). In all sections, the MGB (6 sections per rat), IC (5 sections per rat) and A1 (10 sections per rat) were delineated and the total number of c-Fos positive cells were estimated with the optical fractionator probe. ${ }^{29-31}$ If questionable, boundaries of brain areas were verified in corresponding Nissl stained sections. Established stereological counting methods have been described previously. ${ }^{32}$

\section{Statistical analysis}

Because of our small sample size, non-parametric tests were used for statistical analysis. For analysis of GPIAS test and ABRs, Wilcoxon signed rank tests were performed. For c-Fos, the left and right hemisphere were compared using Wilcoxon signed rank test. The effect of stimulation on the amount of c-Fos positive cells was assessed using Mann Whitney $U$ test. If applicable, HolmBonferroni corrected P-values are presented. P-values $<0.05$ were considered 
significant. All calculations were performed with SPSS (version 22.0 for Mac, SPSS, Chicago, IL, USA).

\section{Results}

\section{Electrode localization}

In all animals, sections where made at the level of DCN and the electrode tips were localized in H\&E stained sections. Exact localization of the electrode tips is presented in Figure 3.2. No histological damage was observed at microscopic level.
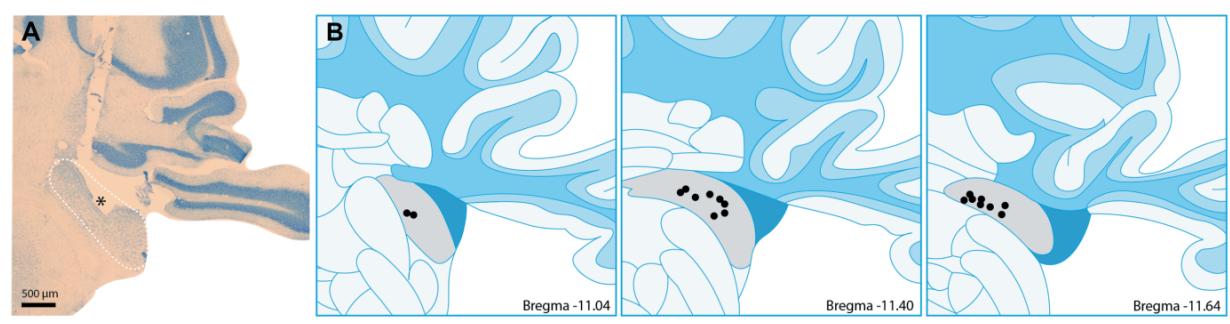

Figure 3.2 A) Representative photomicrograph of a coronal brain section stained for Nissl, showing histological verification of the electrode location (symbol ${ }^{*}$ ) in the dorsal cochlear nucleus (white dotted line). B) Anatomic placement of all electrode tips $($ symbol $\bullet$ ), shown schematically in one hemisphere.

\section{GPIAS}

GPIAS testing results are presented in Figure 3.3. One animal lost the electrode construct and was therefore excluded from GPIAS analysis. Only at $16 \mathrm{kHz}$ background noise, gap:no-gap ratios were significantly increased after noiseexposure $(Z=-2.668, p=0.023)$. This finding is comparable to results in our previous experiments. ${ }^{16,18}$ After noise-exposure, the gap:no-gap ratio decreased during HFS in the $16 \mathrm{kHz}$ background sound $(\mathrm{Z}=-2.310, p=0.042)$. At baseline, there was no effect of HFS ( $p>0.05$ for all frequencies). 


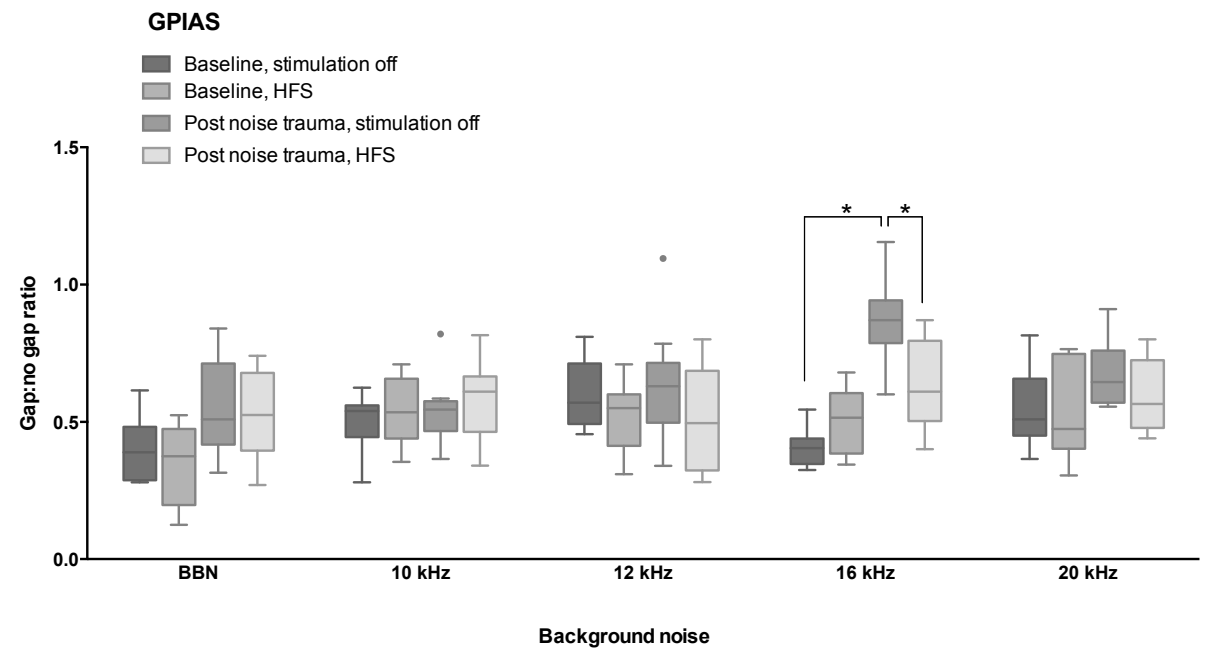

Figure 3.3 Gap-prepulse inhibition of the acoustic startle reflex paradigm for tinnitus assessment before and after exposure to the $16 \mathrm{kHz}$ tone, during stimulation off and HFS. Notice the increased gap:no-gap ratio after noise trauma at $16 \mathrm{kHz}$ background noise. HFS of the DCN caused a decrease of the gap:no-gap ratio. Data are presented as Tukey boxplots. ${ }^{*} p<0.05$

\section{ABR}

In consistency with previous experiments, 17,19 the ABR thresholds (Figure 3.4) were significantly higher after noise trauma compared to baseline in the traumatized (ipsilateral) side along all frequencies $(Z=-2.522, p=0.011, Z=-2.687$, $p=0.007, Z=-2.716, p=0.007, Z=-2.539, p=0.011, Z=-2.716, p=0.007, Z=-2.555$, $p=0.011$, for $10,12,16,20,24,32 \mathrm{kHz}$ respectively). In the contralateral side, hearing thresholds were not increased after noise exposure $(p>0.05$ for all frequencies). Hearing thresholds during HFS could not be determined due to disturbing unspecified artefacts in 13 out of 42 recordings. These recordings were therefore excluded from analyses. HFS did not change hearing thresholds ( $p>0.05$ for all frequencies) in 7 subjects that were recorded during HFS. 


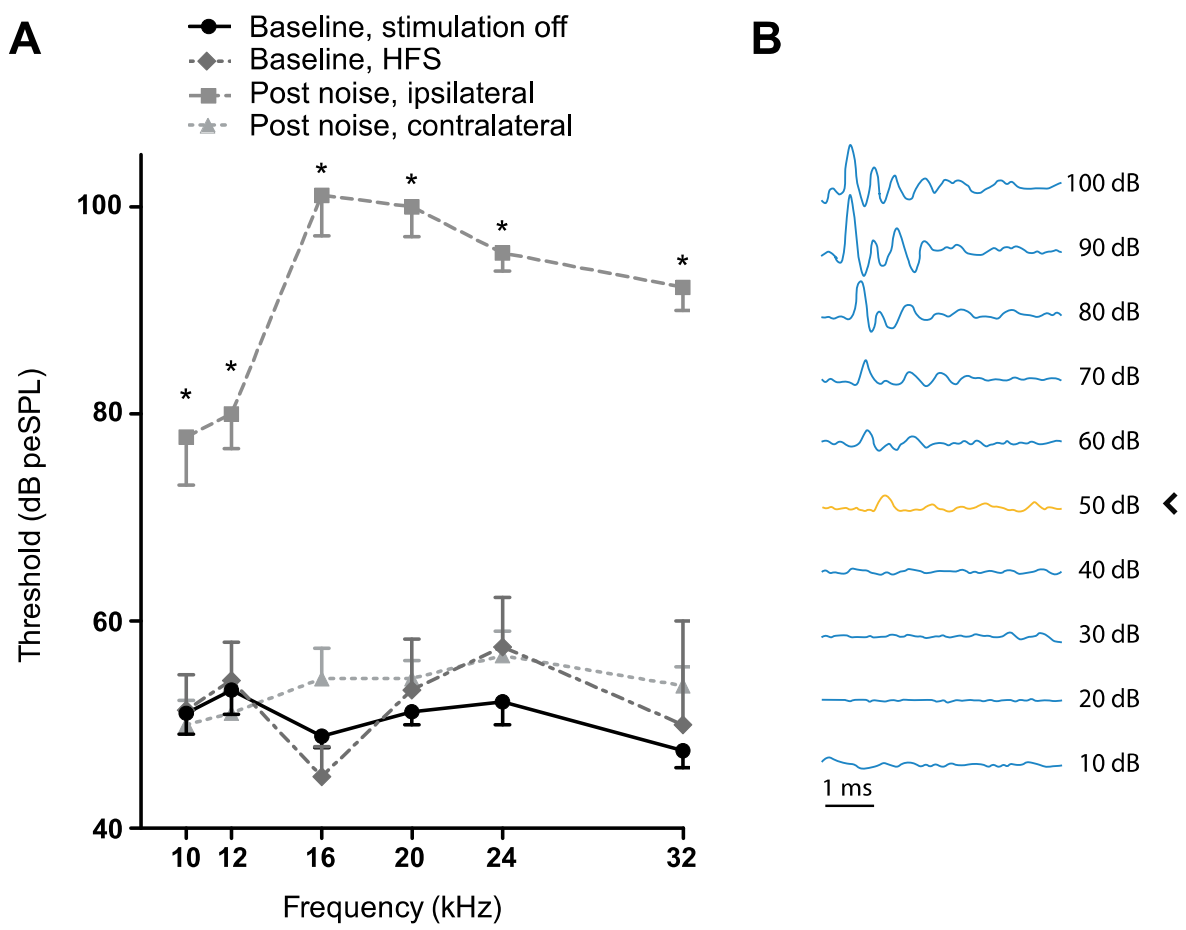

Figure 3.4 A) Auditory thresholds measured with auditory brainstem responses at baseline without HFS (round, solid), during HFS (diamond, dashed-dotted) and after noise exposure in the traumatized ear (square, dashed) and contralateral ear (triangle, dotted). Hearing thresholds at baseline during HFS Auditory thresholds are presented as means +- SEM. * $p<0.05$. B) Example of a recorded Auditory brainstem response with an auditory threshold of $50 \mathrm{~dB}$ peSPL.

\section{C-Fos}

No significant effects of HFS on the total number of c-Fos positive cells in the MGB, IC and A1 ( $p>0.05$ for all areas) were found (Figure 3.5). Noise trauma was applied in all animals in the right ear. There were significantly less c-Fos positive cells in the left compared to right IC in both sham $(Z=-2.023, p=0.043)$ and stimulated $(Z=-2.201, p=0.028)$ groups. In the MGB, there was less $c-F o s$ expression in the right side in only the sham group $(Z=-2.023, p=0.043)$. In $A 1$, there was no difference between left and right in sham $(Z=-0.674, p=0.500)$ and stimulated $(Z=-1.782, p=0.075)$ groups. 
A $\square$ Stimulation off
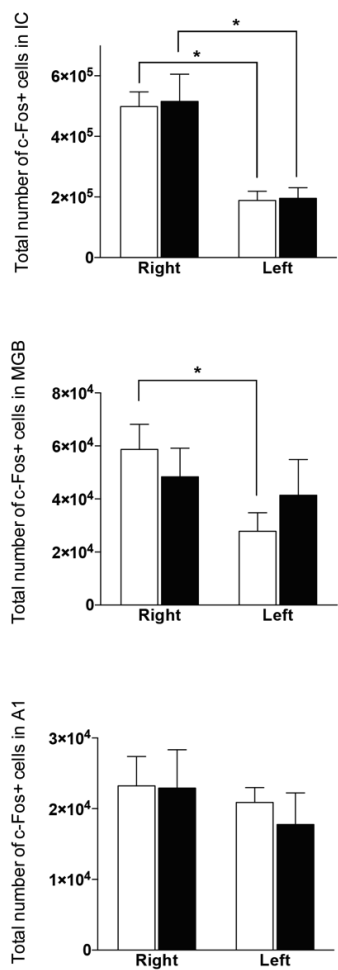

B
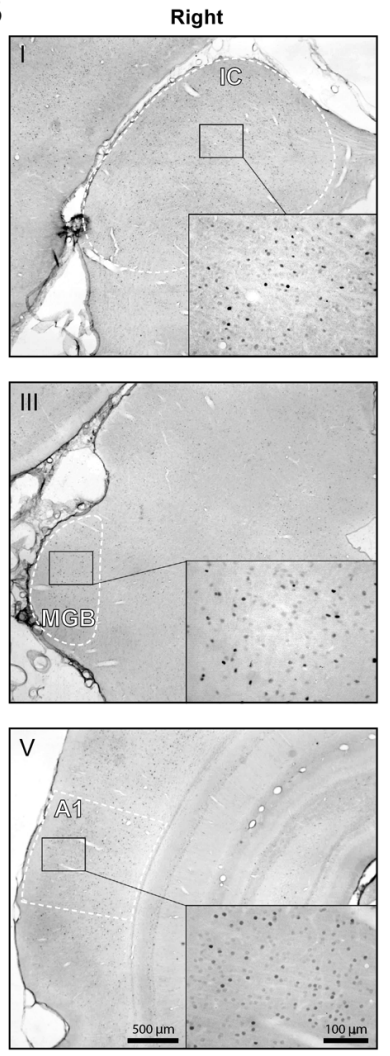
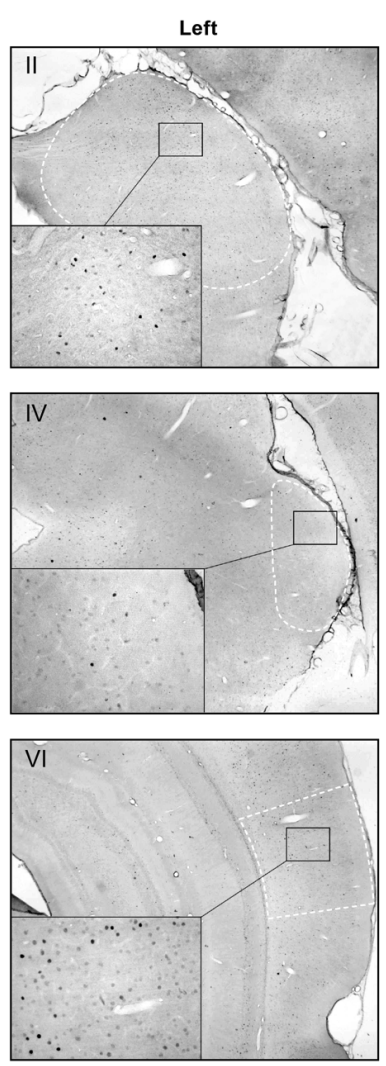

Figure 3.5 Effect of HFS on c-Fos neural activity. A) Total number of c-Fos positive cells in the IC, MGB and A1 in both hemispheres of sham (stimulation off) and HFS groups. Data are presented as mean \pm SEM. B) Representative micrographs of c-Fos immunohistochemical staining of 30- $\mu$ m-thick sections of a non-stimulated animal, showing the IC (I-II), MGB (III-IV) and A1 (V-VI) in both hemispheres. IC = inferior colliculus, MGB = medial geniculate body of the thalamus, $\mathrm{A} 1$ = primary auditory cortex, HFS = high-frequency stimulation.

\section{Discussion}

In the present study we demonstrated that bilateral HFS of the DCN suppressed tinnitus-like behaviour in a noise-exposed animal model of tinnitus. HFS did not increase ABR hearing thresholds. There was no effect of HFS on neural activity within the IC, MGB and A1, measured by c-Fos 
immunoreactivity. Furthermore, unilateral noise-exposure was associated with a contralateral decrease in c-Fos expression in the IC and MGB, but not in A1.

\section{The effect of HFS on neuronal activation}

Neuronal expression of the immediate early gene c-Fos is a valuable marker of brain activity with cellular resolution. Previously, it has been shown that electrical stimulation of the cochlea with $50 \mathrm{~Hz}$ as well as acoustic stimulation causes an increase in Fos expression in the central auditory system, especially in neonatally deafened rats. ${ }^{33}$ These results suggest that cochlear and acoustic stimulation restore input and could herewith generate sound perception. In this study, HFS-DCN did not induce any changes in c-Fos expression in the IC, MGB and A1. Thus, the effect of DCN-HFS cannot be explained by a simple restoration of the lack of input or an inhibitory or excitatory effect on neuronal activity in the network. More likely, DCN-HFS disrupts pathological patterns of activity or temporal coherence, which results in restoration of information flow and blockage of the relay of abnormal signals. However, it should be noted that c-Fos alone might not be a reliable readout, since it could be that changes in cell activity take place during HFS while overall c-Fos activation remain constant.

\section{Electrical stimulation of the central auditory pathway for tinnitus suppression}

Here, we showed a beneficial effect of HFS-DCN on tinnitus-like behaviour. The effect of the prepulse gap on the startle response was restored during HFS at the believed tinnitus-pitch, which was the $16 \mathrm{kHz}$ background sound. In a clinical study in neurofibromatosis type 2 patients, direct electrical stimulation of the DCN with ABI suppresses tinnitus in 6 out of 7 patients who used the device on a daily basis. ${ }^{20}$ The electrical stimuli of ABI are analogues of acoustic waveforms, band-pass filtered between 250 and $4000 \mathrm{~Hz}$, with the purpose to restore hearing. The induced electrical activity however, seems to reduce tinnitus as well. Despite these encouraging results, ABIs are still not used to suppress tinnitus. It remains questionable if and how the results of this study can be extrapolated to potential clinical use of electrical DCN stimulation for tinnitus suppression. First, only one patient experienced complete suppression of tinnitus. Second, the patient group in this study was generally not representative for patients with severe refractory tinnitus. These particular 
patients did not have a functioning auditory nerve and the primary goal of the $\mathrm{ABI}$ was to restore auditory sensations, not to suppress tinnitus.

A beneficial effect of DBS in the DCN on tinnitus-like-behaviour has been confirmed in a noise-exposed rat model for tinnitus. ${ }^{34}$ However, in this preclinical study, a low-frequency stimulation paradigm of $10 \mathrm{~Hz}$ was used and mechanisms underlying this suppression remain unclear. Previously, we have reported that HFS of the IC and MGB suppresses tinnitus in rats as well. ${ }^{17,19}$ Interestingly, we did not find beneficial effects of low-frequency stimulation of the MGB in tinnitus. As far as we are aware, the positive effect of HFS of the DCN on tinnitus has not been reported before.

The positive effect of HFS in three different targets within the classical auditory pathway on tinnitus-like behaviour is noteworthy. Interestingly, in Parkinson's disease a benificial effect can also be obtained when different targets within the cortico-basal ganglia thalamic loop are stimulated. ${ }^{35,36}$ It can be postulated that tinnitus is also a network disorder and disrupting the pathological information flow within the auditory network at any point may suppress tinnitus. ${ }^{18}$ The exact mechanisms behind the effects of high frequency brain stimulation remain unclear. Current research has revealed that the effect of HFS-DBS is beyond simple excitation or inhibition of the local neural elements at the stimulation target. These mechanisms are rather multifactorial and include immediate neuromodulatory effects, synaptic plasticity and long-term neuronal reorganization. ${ }^{37}$

Up to now, it remains uncertain which subcortical target can be stimulated with the least side-effects and risks, and on the other hand best tinnitus reduction. Based on the results in this study, DCN-HFS might have potential to suppress tinnitus loudness and could therefore be a therapeutic option to treat tinnitus. Considering a bottom-up approach, the DCN is the first central region that can be stimulated. Therefore, stimulation of this area might hypothetically have a higher potential to suppress pathological activity since it is less modulated in comparison to more upstream targets. However, it has been suggested that with time hyperexcitability increases in central neurons, which eventually leads to spontaneous firing becoming intrinsically generated in more upstream nuclei. ${ }^{38}$ Contemplating this "progressive centralization" and the undetermined rostral effects of DCN-HFS, it is uncertain if DCN stimulation also has a positive effect on long standing tinnitus. 
The surgical approach to the DCN is not straightforward. The DCN has been stimulated in humans before with an ABI to improve hearing function. This surgical procedure is invasive and complex, and to date has only been performed in patients without a functioning auditory nerve. From a neurosurgical point of view, the MGB of the thalamus is best accessible via stereotaxy and might therefore be the target of first choice. DCN modulation should not be ruled out as a treatment option, as fast progress is made in the development of non-invasive neuromodulation techniques. Examples are focussed ultrasound ${ }^{39}$ and magnetothermal stimulation. ${ }^{40}$

\section{The effect of unilateral noise trauma on neuronal activity and hearing}

In all subjects, hearing thresholds were increased unilaterally after acoustic over-exposure in the right ear. A decrease in c-Fos expression was found in the left compared to right IC (both groups) and MGB (sham group), but not in A1. A lack of input due to hearing loss can cause a lower baseline c-Fos. ${ }^{41,42}$ C-Fos is a general marker for neuronal activity and a decrease in c-Fos expression can both mean a reduction in inhibitory or excitatory neurotransmission. Interestingly, a left-right difference is not found in A1. This finding is in concordance with the results of a study in which they only found a short-term effect of hearing loss on c-Fos expression, but no long-term effect. ${ }^{42}$ There is a relative increased c-Fos expression on the traumatized side, which might reflect increased spontaneous activity in A1. It is thought that tinnitus related activation arises within the thalamocortical loop of the auditory system, ultimately leading to increased spontaneous activity in auditory cortices, more so than in down-stream structures of the auditory pathway. ${ }^{43} \mathrm{~A} 1$ is a more upstream structure, in which the effects of noise trauma are more modulated, due to connectivity with other brain regions. This makes subcortical structures more suitable for neuromodulation. A limitation of this study is that there was no healthy control group, which makes it impossible to compare to the immunohistochemical findings to a healthy state.

The potential confounding effect of hearing loss cannot be ruled out in this study. However, it should be noted that gap:no-gap ratios were only found in the $16 \mathrm{kHz}$ background frequency while unilateral hearing thresholds were increased in all frequency bands. These findings suggest that increased gap:nogap ratios reflect actual tinnitus instead of a hearing-loss induced temporal processing deficit. 


\section{Hearing and DCN-HFS}

A significant concern when stimulating the central auditory pathway is the effect on hearing. In our study we showed that there is no deterioration of hearing during HFS, as assessed with ABR. A previous study has shown that bilateral DCN lesions did not increase hearing thresholds as assessed with ABR. ${ }^{8}$ It seems that a functional DCN is not obligatory for normal hearing thresholds. Furthermore, it has been shown that HFS of other auditory brain structures such as the IC seems not to impair hearing in rats. ${ }^{28}$

It is known from clinical studies that electrical stimulation of the $\mathrm{DCN}^{44}$ and $\mathrm{IC}^{45}$ is able to induce auditory perceptions, on which basically the function of auditory brainstem and midbrain implants rely on. Electrical stimulation of the DCN with low frequency pulses (10 pps) induces neural activation in auditory cortex. ${ }^{46}$ It is unknown if stimulation with continuous HFS will cause auditory perceptions as well.

\section{Conclusion}

Neuromodulation is a promising treatment modality to alleviate tinnitus. Here, we show that DCN-HFS reduces tinnitus-like behaviour in an animal model without increasing ABR hearing thresholds. If seen as a network disorder, the DCN might be the first station that can be targeted with HFS to disrupt the pathological tinnitus signal. Although DCN-HFS is effective, many questions remain unanswered. We therefore encourage neuroscientist, medical specialists and audiologist to further explore neuromodulation for treatment of tinnitus both pre-clinically and clinically. 


\section{References}

1. Norena AJ, Eggermont JJ. Changes in spontaneous neural activity immediately after an acoustic trauma: implications for neural correlates of tinnitus. Hear Res. 2003;183(1-2): 137-153.

2. Norena AJ, Farley BJ. Tinnitus-related neural activity: theories of generation, propagation, and centralization. Hear Res. 2013;295:161-171.

3. Roberts LE, et al. Ringing ears: the neuroscience of tinnitus. J Neurosci. 2010;30(45): 14972-14979.

4. Kaltenbach JA. Tinnitus: Models and mechanisms. Hear Res. 2011;276(1-2):52-60.

5. Kaltenbach JA. Summary of evidence pointing to a role of the dorsal cochlear nucleus in the etiology of tinnitus. Acta Otolaryngol Suppl. 2006;(556):20-26.

6. Kaltenbach JA, Godfrey DA. Dorsal cochlear nucleus hyperactivity and tinnitus: are they related? Am J Audiol. 2008;17(2):S148-S161.

7. Wu C, Martel DT, Shore SE. Increased Synchrony and Bursting of Dorsal Cochlear Nucleus Fusiform Cells Correlate with Tinnitus. J Neurosci. 2016;36(6):2068-2073.

8. Brozoski TJ, et al. Bilateral dorsal cochlear nucleus lesions prevent acoustic-trauma induced tinnitus in an animal model. J Assoc Res Otolaryngol. 2012;13(1):55-66.

9. Brozoski TJ, Bauer CA. The effect of dorsal cochlear nucleus ablation on tinnitus in rats. Hear Res. 2005;206(1-2):227-236.

10. Kaltenbach JA. The dorsal cochlear nucleus as a contributor to tinnitus: mechanisms underlying the induction of hyperactivity. Prog Brain Res. 2007;166:89-106.

11. Bauer CA, et al. Tinnitus and inferior colliculus activity in chinchillas related to three distinct patterns of cochlear trauma. J Neurosci Res. 2008;86(11):2564-2578.

12. Luo $\mathrm{H}$, et al. Blast-induced tinnitus and spontaneous activity changes in the rat inferior colliculus. Neurosci Lett. 2014;580:47-51.

13. Ropp TJ, et al. Effects of unilateral acoustic trauma on tinnitus-related spontaneous activity in the inferior colliculus. J Assoc Res Otolaryngol. 2014;15(6):1007-1022.

14. Kalappa BI, et al. Single unit hyperactivity and bursting in the auditory thalamus of awake rats directly correlates with behavioural evidence of tinnitus. J Physiol. 2014;592(22): 5065-5078.

15. Luo H, Pace E, Zhang J. Blast-induced tinnitus and hyperactivity in the auditory cortex of rats. Neuroscience. 2017;340:515-520.

16. Norena AJ, et al. Neural changes in the auditory cortex of awake guinea pigs after two tinnitus inducers: salicylate and acoustic trauma. Neuroscience. 2010;166(4):1194-1209.

17. Smit JV, et al. Deep brain stimulation of the inferior colliculus in the rodent suppresses tinnitus. Brain Res. 2016;1650:118-124.

18. van Zwieten G, et al. Tinnitus: Is there a place for brain stimulation? Surg Neurol Int. 2016; 7(Suppl 4):S125-S129.

19. van Zwieten G, et al. Inhibition of Experimental Tinnitus with HIgh Frequency Stimulation of the Rat Medial Geniculate Body. Neuromodulation. 2019;22(4):416-424.

20. Soussi T, Otto SR. Effects of electrical brainstem stimulation on tinnitus. Acta Otolaryngol. 1994;114(2):135-140.

21. McConnell GC, et al. Effective deep brain stimulation suppresses low-frequency network oscillations in the basal ganglia by regularizing neural firing patterns. J Neurosci. 2012; 32(45):15657-15668.

22. McIntyre CC, Hahn PJ. Network perspectives on the mechanisms of deep brain stimulation. Neurobiol Dis. 2010;38(3):329-337.

23. Tyler RS, et al. Electrical Stimulation of the Cochlea to Reduce Tinnitus. Semin Hear. 2008; 29(4):326-332.

24. Rubinstein JT, et al. Electrical suppression of tinnitus with high-rate pulse trains. Otol Neurotol. 2003;24(3):478-485. 
25. Whitmer D, et al. High frequency deep brain stimulation attenuates subthalamic and cortical rhythms in Parkinson's disease. Front Hum Neurosci. 2012;6:155.

26. Tan S, et al. Experimental deep brain stimulation in animal models. Neurosurgery. 2010; 67(4):1073-1079; discussion1080.

27. Paxinos GW, C, The rat brain in stereotaxic coordinates: hard cover edition. 2006, New York: Academic press.

28. Smit JV, et al. Hearing assessment during deep brain stimulation of the central nucleus of the inferior colliculus and dentate cerebellar nucleus in rat. PeerJ. 2017;5:e3892.

29. West MJ, Slomianka L, Gundersen HJ. Unbiased stereological estimation of the total number of neurons in thesubdivisions of the rat hippocampus using the optical fractionator. Anat Rec. 1991;231(4):482-497.

30. Schmitz C, Hof PR. Design-based stereology in neuroscience. Neuroscience. 2005;130(4): 813-831.

31. Schmitz C, Hof PR. Recommendations for straightforward and rigorous methods of counting neurons based on a computer simulation approach. J Chem Neuroanat. 2000;20(1):93-114.

32. Temel Y, et al. Protection of nigral cell death by bilateral subthalamic nucleus stimulation. Brain Res. 2006;1120(1):100-105.

33. Jakob TF, Doring U, Illing RB. The pattern of Fos expression in the rat auditory brainstem changes with the temporal structure of binaural electrical intracochlear stimulation. Exp Neurol. 2015;266:55-67.

34. Luo $\mathrm{H}$, et al. Tinnitus suppression by electrical stimulation of the rat dorsal cochlear nucleus. Neurosci Lett. 2012;522(1):16-20.

36. Vitek JL. Mechanisms of deep brain stimulation: excitation or inhibition. Mov Disord. 2002; 17 Suppl 3:S69-72.

35. Chiken S, Nambu A. Disrupting neuronal transmission: mechanism of DBS? Front Syst Neurosci. 2014;8:33.

37. Ashkan K, et al. Insights into the mechanisms of deep brain stimulation. Nat Rev Neurol. 2017;13(9):548-554.

38. Mulders $\mathrm{WH}$, Robertson D. Progressive centralization of midbrain hyperactivity after acoustic trauma. Neuroscience. 2011;192:753-760.

39. Lipsman N, et al. MR-guided focused ultrasound thalamotomy for essential tremor: a proofof-concept study. Lancet Neurol. 2013;12(5):462-468.

40. Chen R, et al. Wireless magnetothermal deep brain stimulation. Science. 2015;347(6229): 1477-1480.

42. Pernia M, et al. c-Fos and Arc/Arg3.1 expression in auditory and visual cortices after hearing loss: Evidence of sensory crossmodal reorganization in adult rats. J Comp Neurol. 2017; 525(12):2677-2689.

41. Harrison RV, Negandhi J. Resting neural activity patterns in auditory brainstem and midbrain in conductive hearing loss. Acta Otolaryngol. 2012;132(4):409-414.

43. Wallhausser-Franke E, et al. Expression of c-fos in auditory and non-auditory brain regions of the gerbil after manipulations that induce tinnitus. Exp Brain Res. 2003;153(4):649-654.

44. Schwartz MS, et al. Auditory brainstem implants. Neurotherapeutics. 2008;5(1):128-136.

45. Lim HH, Lenarz M, Lenarz T. Auditory midbrain implant: a review. Trends Amplif. 2009; 13(3):149-180.

46. Zhang J, Zhang X. Electrical stimulation of the dorsal cochlear nucleus induces hearing in rats. Brain Res. 2010;1311:37-50. 


\section{Inhibition of experimental tinnitus with high frequency stimulation of the rat medial geniculate body}

Van Zwieten G

Janssen MLF

Smit J

Janssen AML

Roet $\mathrm{M}$

Jahanshahi A

Stokroos RJ

Temel Y

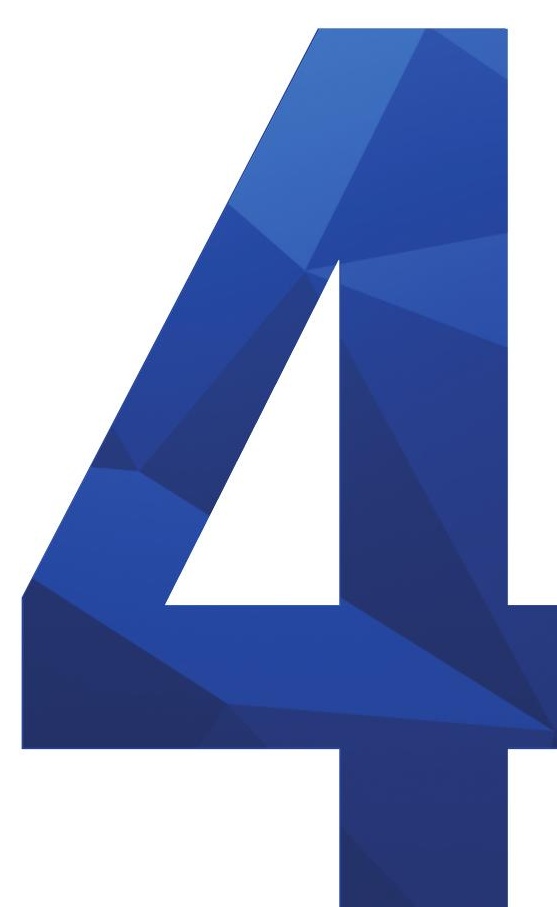




\section{Abstract}

Background: Neuromodulation is a promising treatment modality for tinnitus, especially in chronic and severe cases. The auditory thalamus plays a key role in the pathophysiology of tinnitus, as it integrates and processes auditory and limbic information.

Objective: The effect of high frequency stimulation and low frequency stimulation of the medial geniculate bodies on tinnitus in a noise-induced tinnitus rat model is assessed.

Methods: Presence of tinnitus was verified using the gap induced pre-pulse inhibition of the acoustic startle response paradigm. Hearing thresholds were determined before and after noise trauma with auditory brainstem responses. Anxiety-related side-effects were evaluated in the elevated zero maze and open field.

Results: Results show tinnitus development after noise-trauma and preserved hearing thresholds of the ear that was protected from noise trauma. We found that high frequency stimulation of the medial geniculate bodies suppressed tinnitus. This effect maintained directly after stimulation when the stimulation was turned off. Low frequency stimulation did not have any effects on the gap:no-gap ratio of the acoustic startle response.

Conclusion: High frequency stimulation of the MGB has a direct and residual suppressing effect on tinnitus in this animal model. Low frequency stimulation of the MGB did not inhibit tinnitus. 


\section{Introduction}

Hearing a sound in absence of an audible source is commonly defined as tinnitus. Currently, $2.4 \%$ of the general population suffer from the most severe form of tinnitus, which is often associated with sleeping disorders, anxiety and depression. ${ }^{1,2}$ Even cases of suicide and euthanasia have been reported as a result of the disorder, 3,4 This chronic disorder has a high economic burden on society, ${ }^{5}$ illustrated by the substantial mean annual health care and productivity costs per patient in the Netherlands ( $€ 1544$ and $€ 3702$ respectively). ${ }^{5}$ Although collaborative efforts between multiple specialties have resulted in new therapeutic approaches, tinnitus treatment remains a challenge. ${ }^{6}$

The hypothesis that tinnitus results from pathological increased neural activity in auditory brain structures, most commonly triggered by peripheral input loss, is now widely accepted. ${ }^{7}$ Several models that propose mechanisms leading to tinnitus have been described. Homeostatic plasticity might play an important role. Reduced auditory input results in an increased central neural gain to maintain mean firing rates. ${ }^{8,9}$ Furthermore, breakdown of sensory gating at the level of the medial geniculate body (MGB) of the thalamus might occur..$^{10-12}$ A reduced physiological inhibition could lead to an excitatory/ inhibitory imbalance in auditory nuclei, resulting in a phantom auditory sensation. ${ }^{13,14}$ In a computational model, stochastic resonance is proposed as an underlying mechanism in tinnitus development. Signals are lifted above threshold in order to let them be detected. To facilitate this, hyperactivity is crucial. Through short- and long-term plasticity, this leads to a phantom sound. ${ }^{15}$

The main neural correlates of tinnitus that are observed within the auditory pathway in human and animal studies are increased neural synchrony, tonotopic map changes and increased spontaneous firing. ${ }^{13,16,17}$ Specifically within the MGB, increased spontaneous firing and bursting, and tonotopic reorganization have been described in animals with cochlear damage due to sound exposure, lesioning or ototoxic agents. ${ }^{18-20}$ Besides structures of the auditory pathway, limbic areas play an important role in tinnitus pathophysiology and explain emotional and attentional symptoms of tinnitus. ${ }^{12}$ Deep brain stimulation (DBS) has been proposed as a promising treatment option in severe, refractory tinnitus. ${ }^{21,22}$ Several reports on the effect of DBS in tinnitus have already been reported in humans. ${ }^{23-25}$ However, these are case reports, case series and retrospective data. Preclinical studies have shown that 
DBS of the dorsal cochlear nucleus and inferior colliculus, both auditory structures, resulted in a reduction of tinnitus behavior in rats. 26,27 Of the structures in the hyperactive brain network in tinnitus, the MGB is especially promising as a target for DBS. 22,28 The MGB of the thalamus has an essential gating and shaping function of sensory information and plays a key role in the auditory pathway. At the level of the MGB, limbic and auditory information are integrated. Ascending inputs of the MGB project further to auditory cortices and limbic areas. Descending neurons from auditory and non-auditory cortices contribute to thalamo-cortical loops and further connect to limbic areas, the reticular nucleus and project via the MGB down to the inferior colliculus and dorsal cochlear nucleus. ${ }^{29,30}$ The MGB is part of the thalamus and anatomically accessible using stereotaxy. Furthermore, because the MGB connects the auditory pathway with limbic structures, DBS might alleviate tinnitus loudness as well as the distress accompanied by this phantom sound. ${ }^{22,31}$ The exact working mechanism of DBS remains controversial. ${ }^{32-34}$ High frequency stimulation (HFS) may cause soma inhibition, in combination with axonal activation ${ }^{35}$ and a general hypothesis is that HFS has a complete network effect. ${ }^{31,36}$ Clinically, HFS mimics a lesioning effect. ${ }^{36,37}$ Small patient studies have shown a positive effect of thalamic ablation on tinnitus. ${ }^{38}$ Therefore, we hypothesized that HFS of the MGB results in tinnitus suppression.

Here, we investigated the effects of both HFS and low frequency stimulation (LFS) of the MGB on tinnitus perception. To this aim, a rat model of noise induced tinnitus was used. Auditory brainstem responses were measured before and after noise trauma. Gap-prepulse inhibition of the acoustic startle (GPIAS) response paradigm was used for tinnitus assessment. To test for undesired side-effects, anxiety and general locomotor activity were evaluated with the open field (OF) and elevated zero maze (EZM).

\section{Methods}

\section{Subjects}

Eleven male Sprague Dawley rats (Charles river, Sulzfeld, Germany) were included, weighing approximately $350 \mathrm{~g}$ at time of surgery. All animals were housed individually in standard Makrolon ${ }^{\mathrm{TM}}$ cages (Central Animal Facility of Maastricht University, Maastricht, The Netherlands) to prevent damage to or 
luxation of the electrode construct. Animals were housed in an air-ventilated room under a reversed 12/12 $\mathrm{h}$ light dark cycle with a constant room temperature of $20-22^{\circ} \mathrm{C}$ and a humidity of $60-70 \%$. Standard laboratory chow and water was available ad libitum. All experiments were conducted within the dark period of the day. The experimental protocol was approved by the Animal Experiments and Ethics Committee of Maastricht University. A within subject design was used in order to reduce the error variance, thereby minimizing the number of animals needed.

\section{Overview of study and experimental design}

All animals underwent surgery at the beginning of the experiment. Tinnitus was induced by unilateral noise exposure. Auditory brainstem responses (ABR) were measured before and after noise exposure to estimate hearing levels. The GPIAS response paradigm was used to assess tinnitus perception during four main conditions: 1) baseline stimulation off, 2) baseline stimulation on (HFS), 3) post noise trauma stimulation off and 4) post noise trauma stimulation on, with three different stimulation paradigms. To control for possible confounding (order) effects, measurements were conducted following an incomplete counterbalanced measured design. A schematic overview of the experimental procedures and assessments is shown in Figure 4.1.

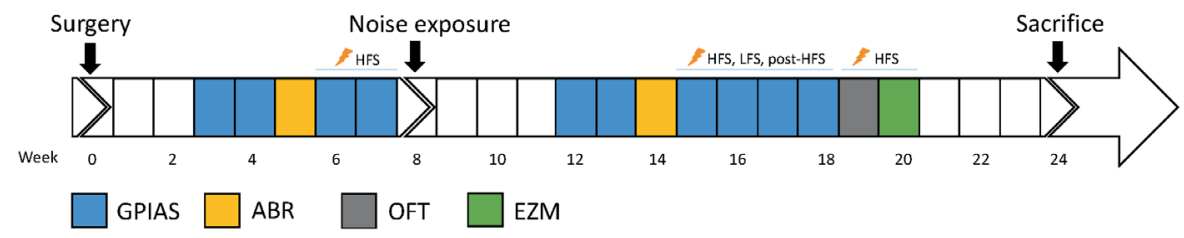

Figure 4.1 Timeline of the experimental procedures. GPIAS $=$ Gap-Prepulse Inhibition of the Acoustic Startle reflex paradigm for tinnitus assessment, ABR $=$ Auditory Brainstem Response recordings, OFT = Open Field Test, EZM = Elevated Zero Maze, HFS = high frequency stimulation, LFS = low frequency stimulation, post-HFS = stimulation off after 30 minutes of high frequency stimulation.

\section{Surgical procedure}

General anesthesia was induced with Xylazin $(10 \mathrm{mg} / \mathrm{kg})$ and Ketamin $(90 \mathrm{mg} / \mathrm{kg})$ i.p. and maintained with Ketamin $(60 \mathrm{mg} / \mathrm{kg} / \mathrm{h})$. Rats were mounted in a stereotactic frame (Stoelting, Wood Dale, IL, USA, model 51,653) with a 
mouth clamp and blunt ear bars in order to prevent damage to the middle ears. DBS electrodes (coaxial gold-coated with platinum-iridium inner wire, shaft diameter of $250 \mu \mathrm{m}$, tip diameter of approximately $50 \mu \mathrm{m}$ ) were bilaterally implanted in the MGB (AP $-5.7 \mathrm{~mm}, \mathrm{ML}+/-3.9 \mathrm{~mm}, \mathrm{DV}-6 \mathrm{~mm}^{39}$ ). The recovery period after surgery was two weeks. Details of the surgical procedure are described elsewhere. ${ }^{40}$

In addition to the DBS stimulation electrodes, two Teflon-coated stainless steel wire electrodes with an exposed tip were implanted for ABR measurements. The recording electrode was subcutaneously tunnelled and placed behind the right mastoid bone, the reference electrode was located at the vertex and secured with a miniature anchoring screw. Permanent electrodes were used to minimize variability between measurements.

\section{Deep brain stimulation}

Rats were tested in the following conditions: (1) stimulation off, meaning attachment to the stimulation cable without electrical stimulation, (2) HFS, which was HFS at $100 \mathrm{~Hz}, 60 \mu$ s pulse width and $100 \mu \mathrm{A}$ amplitude, (3) postHFS, same as paradigm 1 but testing was performed following 30 minutes of HFS and (4) LFS, low frequency stimulation with $10 \mathrm{~Hz}, 60 \mu$ s pulse width and $100 \mu \mathrm{A}$ amplitude. HFS and LFS were applied continuously, from 15 minutes before until the end of the GPIAS response paradigm. Stimulation parameters were chosen based on results of previous DBS experiments. ${ }^{27,41,42}$ Stimulation was bipolar and monophasic pulses were used. The stimulation cable was connected to a constant-current isolator (DLS 100, WPI, Berlin, Germany) which was connected to a stimulator (DS8000, WPI, Berlin, Germany).

\section{Tinnitus induction}

All subjects were unilaterally exposed to a $16 \mathrm{kHz}$ octave-band noise at $115 \mathrm{~dB}$ for 90 minutes (Ultrasonic power amplifier and Ultrasonic Dynamic Speaker Vifa [Avisoft Bioacoustics, Berlin, Germany]), under general anesthesia (see protocol above). The contralateral ear was plugged with clay to prevent hearing loss. After acoustic over-exposure, the subjects were not tested for three weeks. 


\section{Behavioral testing}

Behavioral testing was performed under different stimulation conditions. For GPIAS, subjects were tested for stimulation condition 1 (stimulation off) and 2 (HFS) at baseline and for stimulation conditions 1 (stimulation off), 2 (HFS), 3 (post-HFS) and 4 (LFS) after noise exposure. The EZM and OF were performed 10 and 11 weeks after noise trauma respectively. Since only HFS resulted in therapeutic effects, we evaluated the effects of only HFS in these behavioral tasks. Two stimulation conditions were randomly tested: stimulation off (attachment to the cable) and HFS (30 minutes of continuous HFS before and during testing). The two sessions took place on different days, separated by a two-day washout period.

\section{Gap-Prepulse Inhibition of the Acoustic Startle (GPIAS) response paradigm}

Presence of tinnitus was assessed using the gap detection method. ${ }^{27,43}$ The tests were performed in a sound-attenuating chamber. Subjects were placed inside a cylinder made of vertical aluminum bars and polyethylene floor (diameter $17 \mathrm{~cm}$, height $40 \mathrm{~cm}$ ). The cylinder was placed on a piezo transducer which measured the startle force. A speaker (Ultrasonic Dynamic Speaker Vifa (Avisoft Bioacoustics, Berlin, Germany) was mounted in the ceiling of the testing chamber, $50 \mathrm{~cm}$ above the animal. Sounds were amplified (Ultrasonic power amplifier Avisoft Bioacoustics, Berlin, Germany) and calibrated (Bruel \& Kjaer 2231 decibel meter with a 4191 microphone). The stimulation cable was connected to a swivel in order to allow rotation of the cable.

Background signals in the startle chamber consisted of broadband noise (BBN), or narrow-band noise of $10,12,16$ or $20 \mathrm{kHz}$ at $75 \mathrm{~dB}$. The startle stimulus was a $20 \mathrm{~ms}$ long $115 \mathrm{~dB}$ peak equivalent sound pressure level (peSPL) broadband noise burst. In gap-trials, a silent gap of $50 \mathrm{~ms}$ was inserted $100 \mathrm{~ms}$ prior to the startle stimulus. Each test condition consisted of 20 trials, half of them being gap-trials, presented with a random variable stimulus interval of $20 \pm 5 \mathrm{~s}$. The time to test one GPIAS background frequency was \pm 10 minutes. Prior to every session, each subject acclimatized for 5 minutes in the startle chamber, followed by 10 startle-trials in order to habituate the startle response. The time for one complete session, including all background frequencies, was approximately 60 minutes. The gap:no-gap ratio was calculated for every test condition by dividing the amplitude of each gap-startle by the corresponding 
mean of no-gap startles of that test condition. Responses that contained too disturbing (moving) artifacts were excluded from analysis. For every condition, two GPIAS reflex testing sessions were performed, conducted on separate days. The mean gap:no-gap ratio of the two sessions for every test condition was used for further analysis. All subjects underwent one complete session at the start of the experiment for habituation to the testing procedure, which was not used for analysis. To evaluate the effect of the stimulation paradigms "post-HFS" and "LFS", a shorter GPIAS reflex assessment protocol was used with only $10 \mathrm{kHz}$ and $16 \mathrm{kHz}$ background sounds.

\section{Elevated zero maze (EZM)}

The EZM is a circular runway with a diameter of $98 \mathrm{~cm}$ and a path width of $10 \mathrm{~cm}$, placed $70 \mathrm{~cm}$ above floor level and divided into two open and two enclosed parts with $50 \mathrm{~cm}$ high side walls. Rats were placed on one of the open parts and tracked for 5 minutes using the Ethovision tracking software (Ethovision, Noldus Information Technology, Wageningen, The Netherlands). Movements were recorded and the time spent in open and enclosed parts was calculated.

\section{Open field (OF)}

The OF is a Plexiglas square arena of $100 \times 100 \mathrm{~cm}$ with $40 \mathrm{~cm}$ high clear walls and a dark floor divided into a center of $70 \times 70 \mathrm{~cm}$ with outer surrounding zones of $15 \mathrm{~cm}$ width. Subjects were placed in the center of the arena. During the 10 min trial, the time spent in different zones and the total distance moved was recorded and calculated with Ethovision tracking software (Ethovision, Noldus Information Technology, Wageningen, The Netherlands).

\section{Auditory brainstem response (ABR)}

Subjects were tested under anesthesia (see protocol above) in a soundattenuating Faraday cage. Cables were connected to the sockets of the permanent electrodes on the head of the animal, the ground electrode was connected to the left front paw. 1000 5-ms tone bursts of 10, 12, 16, 20, 24 and $32 \mathrm{kHz}$ and a $\cos ^{2}$ rise and fall filter were created with Matlab and presented unilaterally with a frequency of $50 \mathrm{~Hz}$ at decreasing intensities from 100 to $0 \mathrm{~dB}$ peSPL with steps of $10 \mathrm{~dB}$. The contralateral ear was plugged with clay. Auditory stimuli were calibrated (Bruel \& Kjaer 2231 decibel meter with a 4191 
microphone) and digitally triggered. ABRs were recorded in LabChart Pro 7 (ADInstruments, Castle Hill, Australia) and raw data were imported into Matlab. The evoked responses were amplified 100000 times, band-pass filtered $(300-3000 \mathrm{~Hz})$ and averaged. The auditory threshold was defined as the lowest decibel level (peSPL) of the stimuli that produced a distinctive ABR, in which at least two peaks (positive or negative) had to be clearly visible. ${ }^{27}$

\section{Tissue collections and electrode verification}

At the end of the experiments, all subjects received an overdose of pentobarbital (120-180 mg/kg, i.p.) followed by transcardial perfusion with Tyrode's buffer $(0.1 \mathrm{M})$ followed by fixative containing $4 \%$ paraformaldehyde, $15 \%$ picric acid, and $0.05 \%$ glutaraldehyde in $0.1 \mathrm{M}$ phosphate buffer ( $\mathrm{pH} 7.6)$ at $4^{\circ} \mathrm{C}$. Brains were removed and post-fixed overnight in paraformaldehyde at $4^{\circ} \mathrm{C}$ and subsequently in $1 \% \mathrm{NaN}_{3}$ at $4^{\circ} \mathrm{C}$ for long-term storage. Brains embedded in 10\% gelatin (Sigma-Aldrich, Zwijndrecht, The Netherlands) were cut serially on a vibratome into $30 \mu \mathrm{m}$ thick coronal sections. Sections containing the electrode trajectories were processed for a standard hematoxylin-eosin staining to evaluate the locations of electrode tips.

\section{Statistical analysis}

Normality was checked by the Shapiro-Wilk test and visual inspection of the outcome distributions using histograms and Q-Q plots. A two-way repeated measures analysis of variance (ANOVA) was conducted to investigate the effect of the two-leveled factor 'noise trauma' (before and after noise trauma) and the two-leveled factor 'stimulation' (stimulation off and HFS) on GPIAS. Post-hoc comparisons between different factor levels were made using 2-tailed paired samples $t$-tests for all background frequencies. The effect of the four different stimulation paradigms (stimulation off, HFS, post-HFS and LFS) on GPIAS after noise trauma was analyzed with a one-way repeated measures ANOVA. To assess hearing thresholds after noise trauma a one-way repeated measures ANOVA was performed. Greenhouse-Geisser adjustment was applied to correct against sphericity violations. Effects of HFS on OF and EZM outcomes were evaluated with two-tailed paired samples t-test. Where multiple comparisons were made, the Bonferroni adjusted p-values are given. P-values smaller than 0.05 were considered significant. All calculations were performed with SPSS (version 22.0 for Mac, SPSS, Chicago, IL, USA) and data are presented in mean \pm standard error of the mean (SE). 


\section{Results}

\section{Electrode localization}

All electrode tips were located within the ventral part of the MGB. Exact coordinates of the electrode tips are illustrated in Figure 4.2. Besides the electrode tracts, no additional tissue damage due to electrical stimulation was observed histologically.
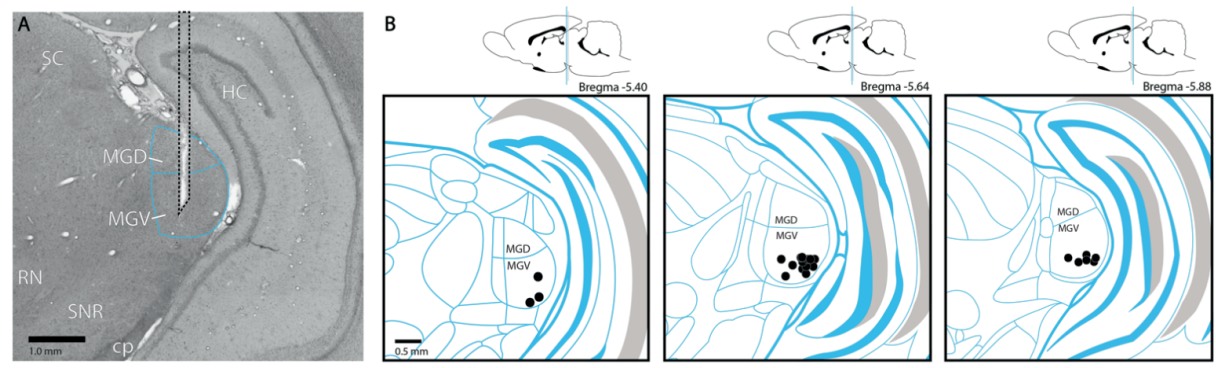

Figure 4.2 A) Representative example of an electrode trajectory in the Medial Geniculate Body. B) Schematic representation of the electrode sites in the Medial Geniculate Body. The symbol $(\bullet)$ indicates the locations of all electrode tips, shown schematically in one hemisphere. MGD = dorsal part of the medial geniculate body, MGV = ventral part of the medial geniculate body, $\mathrm{HC}=$ hippocampus, $\mathrm{SC}=$ superior colliculus, $\mathrm{RN}=$ red nucleus, $\mathrm{SNR}=$ reticular part of substantia nigra, $\mathrm{cp}=$ cerebral peduncle.

\section{Auditory Brainstem Responses}

The ABR thresholds (Figure 4.3) were significantly higher after noise trauma compared to baseline in the ipsilateral (traumatized) side along all frequencies $(t(9)=-5.25, p=0.003, t(9)=-3.85, p=0.008, t(9)=-9.49, p<0.001, t(9)=-5.69, p<0.001$, $t(9)=-6.82, p<0.001, t(9)=-11.00, p<0.001$ for $10,12,16,20,24$ and $32 \mathrm{kHz}$ respectively). Hearing thresholds on the contralateral side were not affected by noise exposure ( $p>0.05$ for all frequencies). ABR measurements were not successful in one rat due to hardware problems, this rat was therefore excluded from hearing threshold analysis. 
- Post noise exposure, ipsilateral

--- Post noise exposure, contralateral

- Baseline

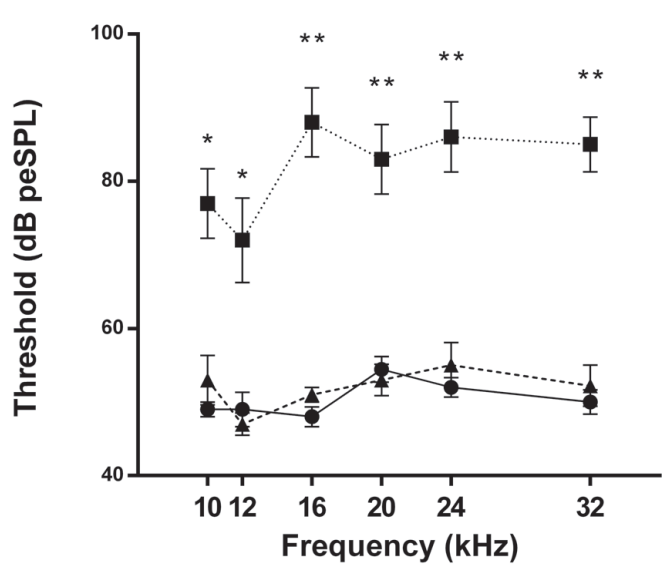

Figure 4.3 Auditory brainstem responses measured at baseline (round, solid) and after noise exposure in the traumatized ear (square, dotted) and the contralateral side (triangle, dashed). Thresholds are presented as means \pm SE. ${ }^{*} p<0.05,{ }^{* *} p<0.001$.

\section{Gap-Prepulse Inhibition of the Acoustic Startle reflex}

Results of GPIAS reflex paradigm are shown in Figure 4.4. Significant main effects have been found for the factors stimulation and noise exposure at $16 \mathrm{kHz}$ background sound $(F(1,10)=22.94, p=0.001$ and $F(1,10)=129.06, p<0.001)$ and $20 \mathrm{kHz} \quad(F(1,10)=9.35, \quad p=0.01$ and $F(1,10)=5.36, \quad p=0.04)$. Significant interaction was found at BBN $(F(1,10)=7.21, p=0.02)$ and $16 \mathrm{kHz}$ background sound $(F(1,10)=76.86, p<0.001)$. Since the power of the interaction test might be insufficient, the simple effects were analysed for all background sounds. Evaluation of these simple effects (Figure 4.4A) showed increased gap:no-gap ratios after noise exposure compared to baseline off-stimulation at $16 \mathrm{kHz}$ (mean difference $0.51(0.04), t(10)=-13.64, p<0.001)$, indicating a reduced detection of the gap-in-noise at this frequency. After noise-trauma, the gap:nogap ratios decreased during HFS in the $16 \mathrm{kHz}$ background sound (mean difference $-0.35(0.05), t(10)=6.56, p<0.001)$ and $20 \mathrm{kHz}(-0.15(0.05), t(10)=3.16$, $p=0.04)$. HFS did not have a significant effect on the gap:no-gap ratios in the baseline situation at any background frequency.

The effect of the three stimulation paradigms (HFS, post-HFS and LFS) compared to stimulation off after noise exposure is illustrated in Figure 4.4B. 
There is a significant main effect $(\mathrm{F}(2.258,22.585)=14.73, p<0.001)$. The gap:nogap ratio significantly decreased at $16 \mathrm{kHz}$ during HFS and post-HFS (with mean differences of $-0.35(0.05), p<0.001)$ and $-0.26(0.05), p=0.002$ respectively), but not during LFS (mean difference of $-0.09(0.05), p=0.095$ ), indicating an increased detection of the gap during HFS and post-HFS at this frequency. Stimulation did not have an effect at $10 \mathrm{kHz}$ background sound.

A
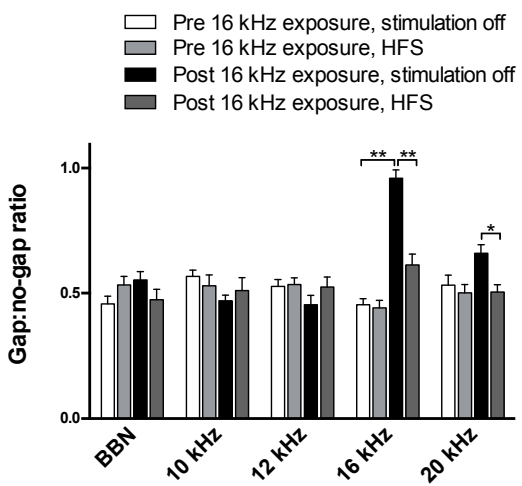

Background
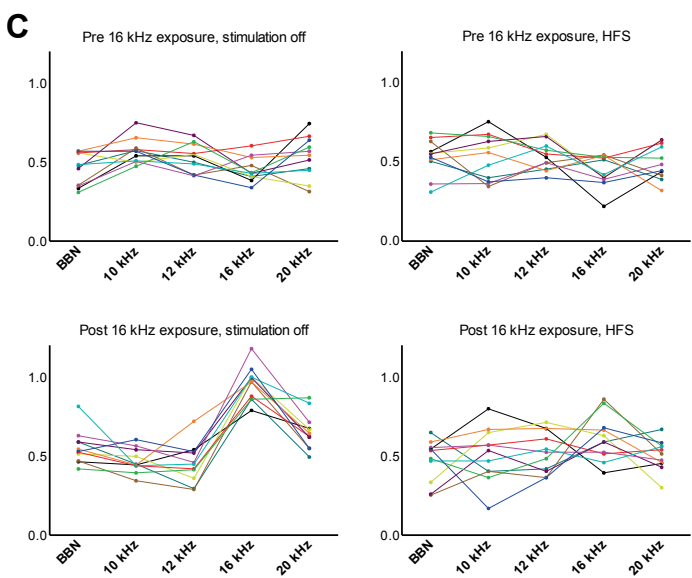

B Post $16 \mathrm{kHz}$ exposure
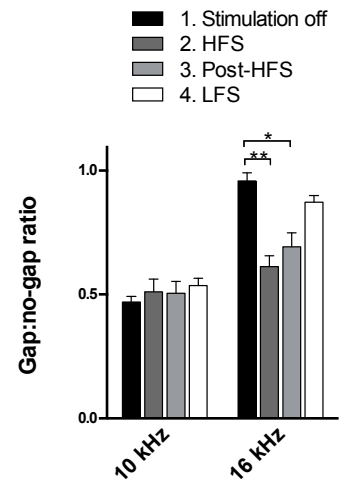

Background

D
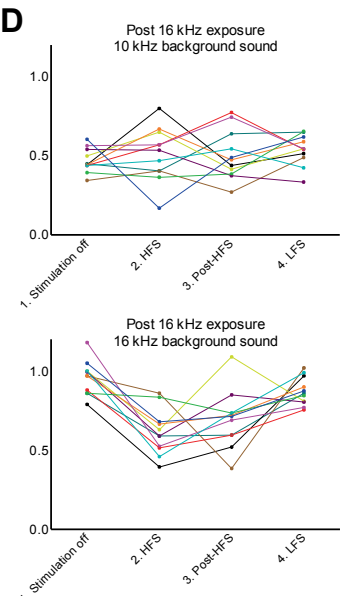

Figure 4.4 A) Gap-prepulse inhibition of the acoustic startle reflex paradigm for tinnitus assessment before and after exposure to the $16 \mathrm{kHz}$ tone, during stimulation off and HFS. Notice the reduced effect of the prepulse gap at $16 \mathrm{kHz}$ background sound on the startle response after noise exposure. During HFS the effect of the prepulse gap was restored at $16 \mathrm{kHz}$ and increased at $20 \mathrm{kHz}$. Gap:no-gap ratios are presented as means \pm SE. Presented significances are simple effects, ${ }^{*} p<0.05^{* *} p<0.001 .$, B) Gapprepulse of the acoustic startle reflex paradigm for tinnitus assessment at $10 \mathrm{kHz}$ and $16 \mathrm{kHz}$ background sound after $16 \mathrm{kHz}$ exposure. Four different stimulation 
paradigms were tested: stimulation off (1), HFS (2), post-HFS (3) and LFS (4). Compared to stimulation off, HFS and post-HFS significantly increased the effect of the gap-prepulse on the acoustic startle response. Gap:no-gap ratios are presented as means \pm SE. ${ }^{*} p<0.05$. ${ }^{* *} p<0.001$. C) Individual gap:no-gap ratios before and after exposure to the $16 \mathrm{kHz}$ tone, during stimulation off and HFS. Each colored line represents one subject. D) Individual gap:no-gap ratios at $10 \mathrm{kHz}$ and $16 \mathrm{kHz}$ background sound after $16 \mathrm{kHz}$ exposure during the four different stimulation paradigms: stimulation off (1), HFS (2), post-HFS (3) and LFS (4). Each colored line represents one subject. BBN $=$ broadband noise, Stimulation off $=$ attached to cable without stimulation, HFS = high frequency stimulation $(100 \mathrm{~Hz}, 60 \mu$ s pulse width, $100 \mu \mathrm{A}$ amplitude), post-HFS = DBS off after 30 minutes of high frequency stimulation, LFS $=$ low frequency stimulation $(10 \mathrm{~Hz}, 60 \mu$ s pulse width, $100 \mu \mathrm{A}$ amplitude).

\section{Elevated zero maze and open field}

HFS did not influence the duration spent in the enclosed arms $(t(10)=-0.11$, $p=0.91)$ or number of entries in the open arms $(t(10)=-0.13, p=0.90)$ of the EZM (Figure 4.5A). There was neither an effect of HFS on the total distance moved $(t(10)=0.29, p=0.78)$ or time spent in corners and/or walls $(t(10)=1.08, p=0.31)$ in the OF (Figure 4.5B).
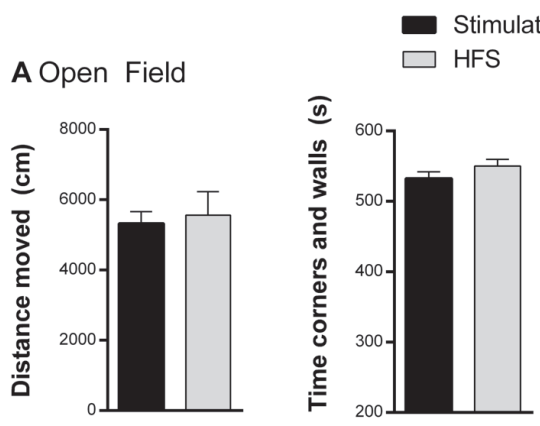

B Elevated Zero Maze
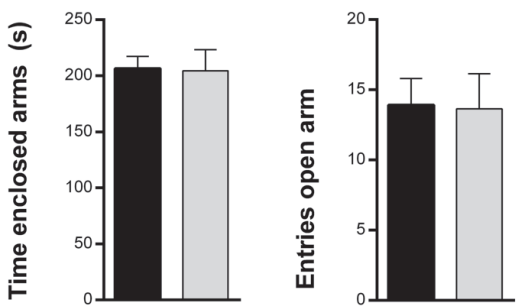

Figure 4.5 A) Number of entries in the open arm and time spent in the enclosed arms (in s) of the elevated zero maze, presented as means $\pm \mathrm{SE}$. There were no significant differences between high frequency stimulation (HFS) and stimulation off. B) Total distance moved (in $\mathrm{cm}$ ) and time spent in corners and walls (in s) in the open field, presented as means \pm SE. There were no significant differences between high frequency stimulation (HFS) and stimulation off. 


\section{Discussion}

In this study, we showed that following noise exposure to induce tinnitus, the prepulse gap had a reduced effect on the startle reflex. Bilateral HFS of the MGB restored this effect. These results suggest that tinnitus can be suppressed with HFS of the MGB. This suppression was only seen when stimulating at a frequency of $100 \mathrm{~Hz}$ and not at a low frequency of $10 \mathrm{~Hz}$. Moreover, no undesired anxiety- or locomotion related side-effects were induced by HFS, assessed in the EZM and OFT.

\section{Tinnitus in the acoustic trauma animal model}

Tinnitus can be assessed in animals using interrogative models or reflexive models. ${ }^{44}$ Interrogative models rely on auditory perception and are therefore believed to mirror actual perception of tinnitus best. By making use of lick or lever pressing suppression or two-choice operant conditioning, rats' perception of certain sounds can be indicated. Training and motivation management is required, and these tests are generally not suitable for longitudinal tinnitus assessment. Reflexive models rely on unconditioned acoustic startle reflexes, which primarily depend on brainstem circuits. ${ }^{44,45}$ The GPIAS reflex paradigm is nowadays the most commonly used method for tinnitus assessment in rodents. ${ }^{44,46}$ The design of this test makes it possible to assess animals' tinnitus over time, which is especially an advantage in our repeated measures design. However, the results obtained by GPIAS test should be carefully interpreted. A relevant limitation of this study is that prepulse inhibition was not performed and absolute startle forces were not obtained. Therefore, it is unsure to what degree the results are influenced by existent hearing loss or hyperacusis in addition to tinnitus. ${ }^{46-49}$

We found increased gap:no-gap ratios in the $16 \mathrm{kHz}$ background sound, which can indicate the presence of tinnitus after unilateral noise trauma. This finding is in accordance with other studies in which a $16 \mathrm{kHz}$ octave band noise also leads to a tinnitus pitch around $16 \mathrm{kHz} \cdot{ }^{27,50}$ In order to prevent loss of implanted electrodes during attachment of the animals to the stimulation cable, we tested all animals while they could freely move in the startle chamber. Startle amplitudes are greatly dependent on the location of the rat on the platform. In order to correct for the variability, group means of the ratios were used in our statistical analysis. All rats showed tinnitus like behavior, as can be 
seen in de individual plotted data. This is a surprising finding, since other studies did not show tinnitus like behavior in all animals. ${ }^{50,51}$

Hearing loss is an important potential confounder. In order to minimize the confounding effect of hearing loss, unilateral noise trauma was applied and hearing thresholds were assessed by ABRs before and after noise trauma. Loss of hearing was found after noise trauma at all frequencies in the traumatized ear, but not in the contralateral ear. While hearing loss was found in all frequency bands, the GPIAS reflex paradigm results only show specific increased gap:no-gap ratios in the $16 \mathrm{kHz}$ background frequency. This indicates that the confounding effect of hearing loss on the GPIAS response is nonessential. Hyperacusis is another potential confounder. Tinnitus and hyperacusis are coexistent in the majority of patients. ${ }^{52}$ Preclinical data suggest hyperacusis and tinnitus to be simultaneously induced after intense sound exposure in animals. ${ }^{50,53}$ It is likely that etiologies are related. ${ }^{48,53}$ However, evidence suggests important differences between the pathophysiological mechanisms in tinnitus and hyperacusis. ${ }^{9}$ Hyperacusis can increase acoustic startle response amplitudes for high-intensity sounds (90 dB SPL or higher) and herewith possibly influence gap:no-gap ratios. ${ }^{48}$ Although we believe specific $16 \mathrm{kHz}$ increased ratios reflect tinnitus-like behavior, it remains unsure to what extent possible confounders like hyperacusis influence the GPIAS. ${ }^{54}$ In order to increase the statistical power, we chose to use a repeated measures design. The risk of order effects is minimized by applying the different stimulation paradigms randomly with substantial time between the conditions (at least 1 day between measurements). To minimize a time effect, we waited 3 weeks with further testing after tinnitus induction. For tinnitus induction, unilateral intense sound exposure was used as this is believed to induce chronic, irreversible symptoms, as opposed to systemic salicylates that have a reversible effect. ${ }^{44,50,55}$

\section{Tinnitus suppression with HFS}

Effects of HFS stimulation, but not of LFS on gap:no-gap ratios were found after noise exposure. During HFS the gap:no-gap ratios significantly decreased at the observed tinnitus pitch $16 \mathrm{kHz}$ and also at $20 \mathrm{kHz}$ frequency bands. Since no effect of HFS on the GPIAS was seen before noise-trauma, the effect of stimulation on the GPIAS results is unlikely to be related to interference from electrical stimulation. 
The hypothesis that specifically HFS of the central auditory pathway might be able to inhibit tinnitus originates from previous studies in Parkinson's disease. The neural correlates associated with Parkinson's disease show similarities with those of tinnitus. In both preclinical and clinical studies, pathological bursting activity and hypersynchrony in the basal ganglia and specifically the subthalamic nucleus is seen in the parkinsonian state. ${ }^{56,57}$ In animal models of tinnitus, similar pathological neurophysiological hallmarks have been found in the MGB and other nuclei within the auditory network.7,13,16,58 Multiple working mechanisms of conventional DBS have been hypothesized, but a uniform theory is still lacking. Theories on changes induced by DBS at the synaptic, axonal, dendritic and neuronal soma exist. Moreover, complete network effects have been investigated. ${ }^{31}$ Conventional DBS at high frequencies leads to inactivation of the neuronal population around the electrode. ${ }^{59} \mathrm{~A}$ proposed mechanism for this inactivation is a depolarization block. ${ }^{60}$ Another mechanism that might play a role, is the stimulation-induced release of GABA from presynaptic terminals. ${ }^{61}$ Besides this, the propagation of action potentials in antero-, retrograde and passing fibers in the vicinity of the electrode is described, which may lead to metabolic and plastic changes in structures at distance and modulate network activity. ${ }^{62}$ Currently, disrupting signaling is key premise of most hypotheses on HFS mechanisms. ${ }^{32,63}$ If HFS is minimal two times higher than the average firing rate of the target neurons, stimulation induced action potentials begin to take over and neurons lose the ability to transmit information. ${ }^{64}$ This "informational lesion" within a circuitopathy, such as described in tinnitus, could eliminate pathological oscillations and normalize neural firing patterns.65-67 This disruptive effect has not been described in LFS, instead, LFS has shown excitatory actions. ${ }^{68}$ These concepts are a reasonable but partial explanation why only HFS and not LFS of the MGB suppressed tinnitus in this study. It is an enormous challenge to unravel the complex question on mechanisms of DBS. It would be of interest to test HFS and LFS in the existing computational models on tinnitus development to further understand the possible working mechanism of DBS in tinnitus. ${ }^{8,15}$

Our results suggest a residual effect of HFS of the MGB on tinnitus suppression. After 30 minutes of HFS, the stimulator was switched off and GPIAS measurements were directly conducted ("post-HFS"). The GPIAS measurement of $16 \mathrm{kHz}$ was finished 10 minutes after electrical stimulation was stopped. Mean gap:no-gap ratios at $16 \mathrm{kHz}$ background sound were significantly lower compared to no stimulation. Further experiments are needed to investigate possible mechanisms and the exact time the remaining 
effect lasts. Electrophysiological techniques could be used to investigate the changes in spontaneous bursting activity and oscillations within the auditory pathway after electrical stimulation of the MGB. A residual effect of electrical stimulation has been repeatedly described in other DBS as well as tinnitus studies. $25,69,70$

\section{Potential side-effects of stimulation}

Limbic and auditory information is integrated at the level of the MGB. ${ }^{10,71}$ Therefore, it can be hypothesized that MGB-HFS could have adverse effects like anxiety. We, however, did not observe any anxiety related behavior during HFS with the stimulation parameters used in this study in two different behavioral paradigms. Theoretically, HFS of the MGB can have an effect on motor behavior, since motor nuclei of the thalamus are located adjacent to the MGB. We neither found any locomotion related side-effects in the OF. Hearing related side-effects are an important concern when electrically stimulating the auditory system. ${ }^{22,73}$ The effect of MGB-HFS on hearing thresholds was not tested in this study. However, in one preclinical study, DBS of the inferior colliculus did not cause impaired hearing thresholds as estimated with sound induced prepulse inhibition. ${ }^{27}$ Generation of unwanted sounds is another potential side effect. This is illustrated by a case of sudden auditory illusions following a small hemorrhagic infarction in the MGB, ${ }^{74}$ which lasted for only 10 minutes. GPIAS response measurements with HFS before noise-trauma did not show significant changes in gap:no-gap ratios, suggesting that HFS does not cause a continuous tinnitus percept at measured background frequencies. However, the GPIAS paradigm is not a validated tool to draw robust conclusions on this matter.

\section{Conclusion}

This study shows first evidence that MGB-HFS suppresses tinnitus in rats. Altogether, our results suggest that specifically HFS (100 Hz) and not LFS (10 $\mathrm{Hz}$ ) of the MGB suppresses tinnitus perception without inducing anxiety and locomotion related side-effects in an experimental rat model of tinnitus. 


\section{References}

1. Axelsson A, Ringdahl A. Tinnitus--a study of its prevalence and characteristics. Br J Audiol. 1989;23(1):53-62.

2. McCormack A, et al. A systematic review of the reporting of tinnitus prevalence and severity. Hear Res. 2016;337:70-79.

3. Lewis JE, Stephens SD, McKenna L. Tinnitus and suicide. Clin Otolaryngol Allied Sci. 1994; 19(1):50-54.

4. Altissimi G, et al. When alarm bells ring: emergency tinnitus. Eur Rev Med Pharmacol Sci. 2016;20(14):2955-2973.

5. Maes IH, et al. Tinnitus: a cost study. Ear Hear. 2013;34(4):508-514.

6. Kreuzer PM, Vielsmeier V, Langguth B. Chronic tinnitus: an interdisciplinary challenge. Dtsch Arztebl Int. 2013;110(16):278-284.

7. Eggermont JJ, Roberts LE. The neuroscience of tinnitus. Trends Neurosci. 2004;27(11):676-682.

8. Schaette R, McAlpine D. Tinnitus with a normal audiogram: physiological evidence for hidden hearing loss and computational model. J Neurosci. 2011;31(38):13452-13457.

9. Knipper M, et al. Advances in the neurobiology of hearing disorders: recent developments regarding the basis of tinnitus and hyperacusis. Prog Neurobiol. 2013;111:17-33.

10. Rauschecker JP, Leaver AM, Muhlau M. Tuning out the noise: limbic-auditory interactions in tinnitus. Neuron. 2010;66(6):819-826.

11. Vanneste $S$, et al. The neural correlates of tinnitus-related distress. Neuroimage. 2010;52(2):470-480.

12. De Ridder D, et al. An integrative model of auditory phantom perception: tinnitus as a unified percept of interacting separable subnetworks. Neurosci Biobehav Rev. 2014;44:16-32.

13. Kaltenbach JA. Tinnitus: Models and mechanisms. Hear Res. 2011;276(1-2):52-60.

14. De Ridder D, et al. Thalamocortical Dysrhythmia: A Theoretical Update in Tinnitus. Front Neurol. 2015;6:124.

15. Krauss P, et al. Stochastic Resonance Controlled Upregulation of Internal Noise after Hearing Loss as a Putative Cause of Tinnitus-Related Neuronal Hyperactivity. Front Neurosci. 2016; 10:597.

16. Roberts LE, et al. Ringing ears: the neuroscience of tinnitus. J Neurosci 2010;30(45): 14972-14979.

17. Norena AJ, Eggermont JJ. Changes in spontaneous neural activity immediately after an acoustic trauma: implications for neural correlates of tinnitus. Hear Res. 2003;183(1-2):137-153.

18. Kalappa BI, et al. Single unit hyperactivity and bursting in the auditory thalamus of awake rats directly correlates with behavioural evidence of tinnitus. J Physiol. 2014;592(22): 5065-5078.

19. Kamke MR, Brown M, Irvine DR. Plasticity in the tonotopic organization of the medial geniculate body in adult cats following restricted unilateral cochlear lesions. J Comp Neurol. 2003;459(4):355-367.

20. Basta D, et al. Bilateral Changes of Spontaneous Activity Within the Central Auditory Pathway Upon Chronic Unilateral Intracochlear Electrical Stimulation. Otol Neurotol. 2015; 36(10):1759-1765.

21. Yong-bing S, Hal Martin W. Deep Brain Stimulation-A new treatment for tinnitus. Journal of Otology. 2007;2(1):1-6.

22. van Zwieten G, et al. Tinnitus: Is there a place for brain stimulation? Surg Neurol Int. 2016 7(Suppl 4):S125-129.

23. Cheung SW, Larson PS. Tinnitus modulation by deep brain stimulation in locus of caudate neurons (area LC). Neuroscience 2010;169(4):1768-1778.

24. Larson PS, Cheung SW. A stroke of silence: tinnitus suppression following placement of a deep brain stimulation electrode with infarction in area LC. J Neurosurg. 2013;118(1):192-194. 
25. Shi $Y$, et al. Deep brain stimulation effects in patients with tinnitus. Otolaryngol Head Neck Surg. 2009;141(2):285-287.

26. Luo $\mathrm{H}$, et al. Tinnitus suppression by electrical stimulation of the rat dorsal cochlear nucleus. Neurosci Lett. 2012;522(1):16-20.

27. Smit JV, et al, Deep brain stimulation of the inferior colliculus in the rodent suppresses tinnitus. Brain Res. 2016;1650:118-124.

28. Smit JV, et al. Deep brain stimulation in tinnitus: current and future perspectives. Brain Res. 2015;1608:51-65.

29. Rees AP, A.R. The Oxford Handbook of Auditory Science, The Auditory Brain. Vol. 2. 2010, New York: Oxford University Press Inc. 580.

30. Winer JA. Decoding the auditory corticofugal systems. Hear Res. 2005;207(1-2):1-9.

31. McIntyre CC, Hahn PJ. Network perspectives on the mechanisms of deep brain stimulation. Neurobiol Dis. 2010;38(3):329-337.

32. Chiken S, Nambu A. Mechanism of Deep Brain Stimulation: Inhibition, Excitation, or Disruption? Neuroscientist. 2016;22(3):313-322.

33. Dostrovsky JO, Lozano AM. Mechanisms of deep brain stimulation. Mov Disord. 2002;17 Suppl 3:S63-68.

34. Vitek JL. Mechanisms of deep brain stimulation: excitation or inhibition. Mov Disord. 2002;17 Suppl 3:S69-72.

35. McIntyre CC, et al. Cellular effects of deep brain stimulation: model-based analysis of activation and inhibition. J Neurophysiol. 2004;91(4):1457-1469.

36. Benabid AL, et al. Chronic electrical stimulation of the ventralis intermedius nucleus of the thalamus as a treatment of movement disorders. J Neurosurg. 1996;84(2):203-214.

37. Benabid AL, et al. Acute and long-term effects of subthalamic nucleus stimulation in Parkinson's disease. Stereotact Funct Neurosurg. 1994;62(1-4):76-84.

38. Jeanmonod D, Magnin M, Morel A. Chronic neurogenic pain and the medial thalamotomy. Schweiz Rundsch Med Prax. 1994;83(23):702-707.

39. Paxinos GW, C. The rat brain in stereotaxic coordinates: hard cover edition. 2006, New York: Academic press.

40. Tan S, et al. Experimental deep brain stimulation in animal models. Neurosurgery. 2010;67(4): 1073-9; discussion1080.

41. Lim LW, et al. The antidepressant effects of ventromedial prefrontal cortex stimulation is associated with neural activation in the medial part of the subthalamic nucleus. Behav Brain Res. 2015;279:17-21.

42. Temel $\mathrm{Y}$, et al. Acute and separate modulation of motor and cognitive performance in parkinsonian rats by bilateral stimulation of the subthalamic nucleus. Exp Neurol. 2005; 193(1):43-52.

43. Turner JG, et al. Gap detection deficits in rats with tinnitus: a potential novel screening tool. Behav Neurosci. 2006;120(1):188-195.

44. Brozoski TJ, Bauer CA. Animal models of tinnitus. Hear Res. 2016;338:88-97.

45. Koch M. The neurobiology of startle. Prog Neurobiol. 1999;59(2):107-128.

46. Galazyuk A, Hebert S. Gap-Prepulse Inhibition of the Acoustic Startle Reflex (GPIAS) for Tinnitus Assessment: Current Status and Future Directions. Front Neurol. 2015;6:88.

47. Heffner HHR. Behavioral Tests for Tinnitus in Animals, in Springer Handbook of Auditory Research. 2012, Springer: New York Heidelberg Dordrecht London.

48. Hayes SH, et al. Behavioral models of tinnitus and hyperacusis in animals. Front Neurol. 2014; 5:179.

49. Lobarinas E, Hayes SH, Allman BL. The gap-startle paradigm for tinnitus screening in animal models: limitations and optimization. Hear Res. 2013;295:150-160.

50. Turner JG, Larsen D. Effects of noise exposure on development of tinnitus and hyperacusis: Prevalence rates 12 months after exposure in middle-aged rats. Hear Res. 2016;334:30-36.

51. Pace E, Zhang J. Noise-induced tinnitus using individualized gap detection analysis and its relationship with hyperacusis, anxiety, and spatial cognition. PLoS One. 2013;8(9):e75011. 
52. Dauman R, Bouscau-Faure F. Assessment and amelioration of hyperacusis in tinnitus patients. Acta Otolaryngol. 2005;125(5):503-509.

53. Chen G, et al. Behavioral evidence for possible simultaneous induction of hyperacusis and tinnitus following intense sound exposure. J Assoc Res Otolaryngol. 2013;14(3):413-424.

54. Eggermont JJ, Roberts LE. The neuroscience of tinnitus: understanding abnormal and normal auditory perception. Front Syst Neurosci. 2012;6:53.

55. Eggermont JJ, Roberts LE. Tinnitus: animal models and findings in humans. Cell Tissue Res. 2015;361(1):311-336.

56. Janssen ML, et al. Mild dopaminergic lesions are accompanied by robust changes in subthalamic nucleus activity. Neurosci Lett. 2012;508(2):101-105.

57. Bergman $\mathrm{H}$, et al. The primate subthalamic nucleus. II. Neuronal activity in the MPTP model of parkinsonism. J Neurophysiol. 1994;72(2):507-520.

58. Basta D, Goetze R, Ernst A. Effects of salicylate application on the spontaneous activity in brain slices of the mouse cochlear nucleus, medial geniculate body and primary auditory cortex. Hear Res. 2008;240(1-2):42-51.

59. Lozano AM, et al. Deep brain stimulation for Parkinson's disease: disrupting the disruption. Lancet Neurol. 2002;1(4):225-231.

60. Florence G, et al. Deep Brain Stimulation: More Complex than the Inhibition of Cells and Excitation of Fibers. Neuroscientist. 2016;22(4):332-345.

61. Moser A, et al. Deep brain stimulation: response to neuronal high frequency stimulation is mediated through GABA(A) receptor activation in rats. Neurosci Lett. 2003;341(1):57-60.

62. Hamani $C$, Temel Y. Deep brain stimulation for psychiatric disease: contributions and validity of animal models. Sci Transl Med. 2012;4(142):142rv8.

63. Grill WM, Snyder AN, Miocinovic S. Deep brain stimulation creates an informational lesion of the stimulated nucleus. Neuroreport. 2004;15(7):1137-1140.

64. McIntyre CC, Anderson RW. Deep brain stimulation mechanisms: the control of network activity via neurochemistry modulation. J Neurochem. 2016;139 Suppl 1:338-345.

65. McConnell GC, et al. Effective deep brain stimulation suppresses low-frequency network oscillations in the basal ganglia by regularizing neural firing patterns. J Neurosci. 2012;32(45): 15657-15668.

66. Whitmer D, et al. High frequency deep brain stimulation attenuates subthalamic and cortical rhythms in Parkinson's disease. Front Hum Neurosci. 2012;6:155.

67. Hamani C, Moro E. Neuromodulation: a more comprehensive concept beyond deep brain stimulation. Int Rev Neurobiol. 2012;107:1-3.

68. Nandi D, et al. The pedunculopontine nucleus in Parkinson's disease: primate studies. Br J Neurosurg. 2008;22 Suppl 1:S4-8.

69. Arts RA, et al. Tinnitus Suppression by Intracochlear Electrical Stimulation in Single-Sided Deafness: A Prospective Clinical Trial - Part I. Audiol Neurootol. 2015;20(5):294-313.

70. Cooper SE, et al. Association of deep brain stimulation washout effects with Parkinson disease duration. JAMA Neurol. 2013;70(1):95-99.

71. Barry KM, et al. Modulation of medial geniculate nucleus neuronal activity by electrical stimulation of the nucleus accumbens. Neuroscience. 2015;308:1-10.

72. Lim HH, et al. The auditory midbrain implant: effects of electrode location. Hear Res. 2008; 242(1-2):74-85.

73. Hazell JW, et al. Electrical tinnitus suppression: frequency dependence of effects. Audiology. 1993;32(1):68-77.

74. Fukutake T, Hattori T. Auditory illusions caused by a small lesion in the right medial geniculate body. Neurology. 1998;51(5):1469-1471. 



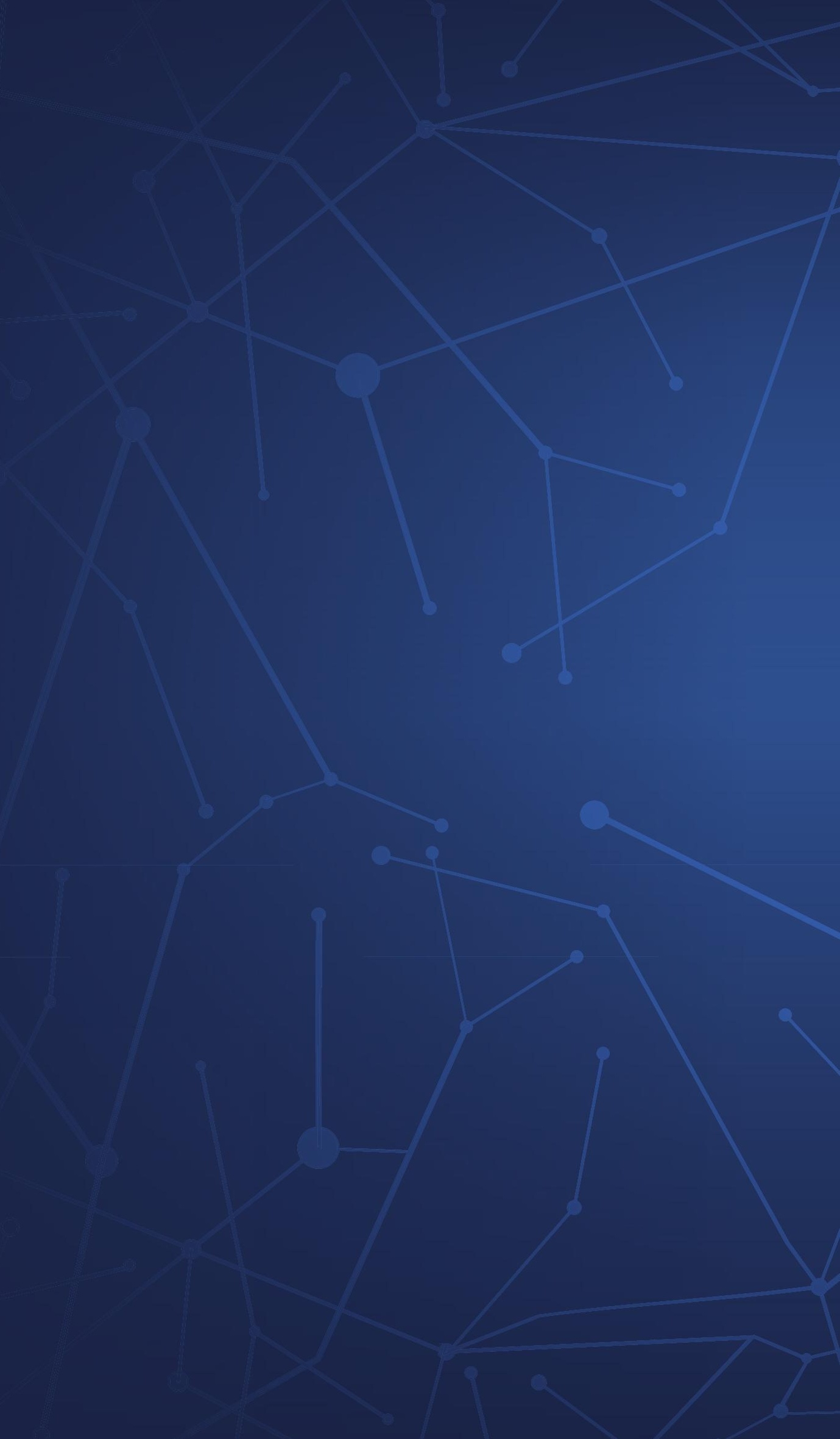




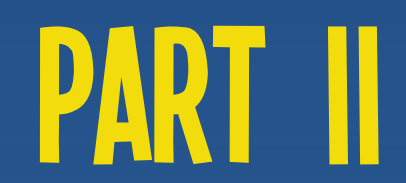

Neuropathophysiological mechanisms 

Noise-induced neurophysiological alterations in the rat medial geniculate body and thalamocortical desynchronization by deep brain stimulation

Van Zwieten G

Roberts MJ

Schaper FLVW

Smit JV

Temel Y

Janssen MLF

Published in Journal of Neurophysiology,

Feb 2021, Volume 125.

DOI: 10.1152/jn.00752.2019 


\section{Abstract}

The thalamic medial geniculate body (MGB) is uniquely positioned within the neural tinnitus networks. Deep brain stimulation (DBS) of the MGB has been proposed as a possible novel treatment for tinnitus, yet mechanisms remain elusive. The aim of this study was to characterize neurophysiologic hallmarks in the MGB after noise-exposure and to assess the neurophysiological effects of electrical stimulation of the MGB. Fourteen male Sprague Dawley rats were included. Nine subjects were unilaterally exposed to a $16 \mathrm{kHz}$ octave-band noise at $115 \mathrm{~dB}$ for 90 minutes, five received sham exposure. Single units were recorded from the contralateral MGB where spontaneous firing, coefficient of variation, response type, rate-level functions and thresholds were determined. Local field potentials and electroencephalographical (EEG) recordings were performed before and after high frequency DBS of the MGB. Thalamocortical synchronization and power were analyzed. In total, 214 single units were identified ( $\mathrm{n}=145$ in noise-exposed group, $\mathrm{n}=69$ in control group). After noiseexposure, fast-responding neurons become less- or non-responsive without change to their spontaneous rate, while sustained and suppressed type neurons exhibit enhanced spontaneous activity without change to their stimulus driven activity. MGB DBS suppressed thalamocortical synchronization in the beta and gamma bands, supporting suppression of thalamocortical synchronization as an underlying mechanism of tinnitus suppression by high frequency DBS. These findings contribute to our understanding of the neurophysiologic consequences of noise-exposure and the mechanism of potential DBS therapy for tinnitus. 


\section{Introduction}

Acoustic overexposure is the most common cause of tinnitus. ${ }^{1}$ Patients suffering from tinnitus perceive a phantom sound. To date, the exact pathophysiological mechanisms involved in tinnitus are still poorly understood. According to the most accepted theory, tinnitus is generated in response to a reduction of input from the cochlea to the brain. Even though tinnitus often arises after hearing loss, persons with hearing loss do not always develop tinnitus. Therefore, it has been postulated that not only bottom-up deafferentation, but also a deficient top-down noise-cancelling mechanism plays a role in tinnitus development. ${ }^{2,3}$ Overall, a hyperactive state is found within tinnitus related brain structures, characterized by increased spontaneous and bursting activity, tonotopic reorganization and enhanced local and long-range neural synchrony. ${ }^{4-6}$

The medial geniculate body (MGB) is part of the auditory thalamus and has a unique position in auditory pathophysiology.7 This structure acts as a central hub in purported tinnitus networks, as it connects to both auditory and limbic structures. ${ }^{2}$ It has been suggested that the MGB plays a significant role in not only the auditory, but also the emotional and attentional aspects of tinnitus. ${ }^{8,9}$ MGB neurons integrate and shape neural representations of auditory information and control passage to the cerebral cortex. ${ }^{10}$ The ventral division of the MGB receives auditory input from the central inferior colliculus, and mainly projects to the primary auditory cortex. The medial and dorsal MGB receives input from dorsal and external cortex of the inferior colliculus, which further projects to belt regions of the auditory cortex. Moreover, paralimbic structures connect via the thalamic reticular nucleus to the MGB, which acts as a thalamic gate and might function as a "noise cancelling system". Two tinnitus models propose essential involvement of the MGB in tinnitus pathophysiology. First, it has been suggested that the inhibitory feedback loop (or thalamic gate) does not work sufficiently in tinnitus patients. ${ }^{2}$ Second, thalamocortical oscillations and increased coherence among high- and lowfrequency oscillations indicate presence of thalamocortical dysrhythmia in tinnitus and might be responsible for the phantom sound. ${ }^{3,11}$

Despite its central position in the auditory network and multiple pathophysiological hypotheses, the MGB has received little attention in neurophysiological studies. While electrophysiological recordings in human 
MGB are unavailable, structural and functional abnormalities in the thalamus have been investigated in tinnitus patients using imaging studies..$^{12} \mathrm{~A}$ highresolution magnetic resonance imaging (MRI) study using voxel-based morphometry showed increased gray-matter volume at the auditory thalamus ${ }^{13}$ relative to healthy participants, although these findings could not be replicated by others. ${ }^{14}$ In a functional MRI study, lateralized activation of MGB was found in unilateral tinnitus, while bilateral tinnitus was associated with bilateral activation pattern. ${ }^{15}$

Thus far, only few MGB related neurophysiological studies in the noiseexposed animal model have been reported. In one study, an increase in spontaneous activity, burst properties and sound-evoked activity of MGB neurons was found in awake rats with behavioral evidence of tinnitus. ${ }^{16}$ In another study, noise-exposed anesthetized tinnitus rats did not show increased spontaneous firing rates but showed a reduction in neurons with bursty firing. ${ }^{17}$ In an in vitro study on mice brain slices, the firing rate of MGB neurons was increased in some neurons and decreased in others after application of salicylate, which is known to induce temporary tinnitus. ${ }^{18}$ In one of our recent studies, MGB DBS was effective in suppressing tinnitus like behavior in a noise-induced animal model. ${ }^{19}$ We therefore propose the MGB as a potential therapeutic target in deep brain stimulation (DBS) for tinnitus. ${ }^{20}$

The aim of this study was to further characterize the neurophysiologic fingerprint of the MGB in the noise-exposed animal model and to assess the neurophysiological effects of electrical stimulation of the MGB. More insight in neuropathophysiological changes after noise-exposure will contribute to further unravel physiological, but also pathological auditory processing such as in case of tinnitus. To this aim, single unit recordings from noise-exposed rodents and unexposed control animals were recorded. To explore the working mechanism of MGB DBS, spontaneous local field potentials (LFP) and electroencephalographical (EEG) recordings were performed before and after application of high frequency DBS in the MGB. 


\section{Materials and methods}

\section{Subjects and experimental design}

Fourteen male Sprague Dawley rats (Charles River, Sulzfeld, Germany) were included in this study, aged 7 weeks and weighing $\pm 250 \mathrm{~g}$ at the start of the experiment. Animals were housed in pairs with ad libitum access to food and water, and with a reversed day/night cycle. Experiments were conducted in the dark period of the day. The experimental protocol was approved by the Animal Experiments and Ethics Committee of Maastricht University. Subjects were divided in two groups: noise exposure $(n=9)$ and control $(n=5)$.

\section{Noise exposure}

All subjects were anesthetized by use of Ketamine $(10 \mathrm{mg} / \mathrm{kg}$ and for maintenance $60 \mathrm{mg} / \mathrm{kg} / \mathrm{h}$ ) and Xylazine $(90 \mathrm{mg} / \mathrm{kg})$. Subjects in the noiseexposed group were unilaterally exposed to a $16 \mathrm{kHz}$ octave-band noise at $115 \mathrm{~dB}$ for 90 minutes (Ultrasonic Power Amplifier and Ultrasonic Dynamic Speaker Vifa; Avisoft Bioacoustics, Berling, Germany), which was calibrated at speaker-level (Bruel \& Kjaer $2231 \mathrm{~dB}$ meter and 4191 microphone). A plug of clay protected the contralateral ear from noise.

\section{Gap-Prepulse Inhibition of Acoustic Startles}

Behavioral testing of tinnitus was conducted using gap-prepulse inhibition of acoustic startle response paradigm (GPIAS, detailed description has been described elsewhere $\left.{ }^{21}\right)$. All subjects were tested in two conditions: baseline, and 3 weeks after noise- or sham-exposure. Briefly, rats were placed inside a cylinder with vertical aluminum bars and a polyethylene floor (diameter $17 \mathrm{~cm}$, height $40 \mathrm{~cm}$ ), inside an acoustic chamber. Calibrated auditory stimuli were amplified and presented via a speaker (Ultrasonic Power Amplifier and Ultrasonic Dynamic Speaker Vifa; Avisoft Bioacoustics, Berling, Germany), which was centrally located in the ceiling of the chamber. Background signals consisted of narrow-band noise of 10,16 and $20 \mathrm{kHz}$ at $75 \mathrm{~dB}$. The startle stimulus was a $20 \mathrm{~ms}$ long, $115 \mathrm{~dB}$ equivalent sound pressure level broadband noise (BBN) burst. A silent gap of $50 \mathrm{~ms}$ was embedded in the background noise, $100 \mathrm{~ms}$ prior to the startle stimulus in all gap-trials. One session consisted of 10 gap trials and 10 startle-only trials for every background sound. Prior to each session, subjects were acclimatized for 5 minutes and habituated 
by presenting 10 startle-only trials. To calculate the gap:no gap ratios, amplitude of each gap-startle was divided by the corresponding mean of nogap startles. Two complete sessions per condition were performed for each subject on separate consecutive days. One additional complete session was performed at the start of the experiment to habituate the animals to the testing procedure.

\section{Single unit recordings}

Electrophysiological measurements were conducted four to five weeks after noise exposure. The order of the experiments in the two study groups was randomized. Rats were anesthetized with urethane $(1.5 \mathrm{~g} / \mathrm{kg}$, i.p.) and fixed in a stereotactic frame (model 51950, Stoelting Co., Wood Dale, USA) with hollow ear bars (World Precision Instruments, Sarasota, USA) to allow presentation of auditory stimuli. Body temperature was controlled and maintained at 37 degrees with a heating pad (ATC1000, World Precision Instruments, Sarasota, USA). To allow access to the MGB, a small craniotomy and durotomy was performed contralateral to the side of noise exposure at identified coordinates. ${ }^{22}$ A glass microelectrode, pulled from Kwik-Fil (World Precision Instruments, Sarasota, USA) filamented borosilicate glass capillaries with a tip diameter of $1 \mu \mathrm{m}$, containing $0.9 \% \mathrm{NaCl}$, was then carefully lowered into the MGB (AP -5.4-5.8 mm, ML 3.4-3.5 mm relative to Bregma, DV 3.8-6.8 mm from dura) using a hydrolic drum Microdrive (FHC, Bowdoin, USA). The electrode was connected to an AlphaMap data acquisition system (AlphaOmega, Nazareth, Israel), allowing recording of extracellular neuronal activity (sampling rate $25 \mathrm{kHz}$, high pass filter $350 \mathrm{~Hz}$, low pass filter $5 \mathrm{kHz}$ ). A sound paradigm was designed using a custom-made Matlab script (V2015A; Mathworks inc, Natick, USA). Sounds were processed with an external soundcard (E-MU 0204, Creative Technology Ltd, Singapore) with a sampling rate of $192 \mathrm{kHz}$ and amplified using an Ultrasonic power amplifier and Ultrasonic Dynamic Speaker Vifa (Avisoft Bioacoustics, Berlin, Germany). Whilst lowering the electrode, search stimuli (200 ms BBN, 2 presentations per second) were played. Lowering was stopped each time a well isolated spike was observed. A standardized paradigm of auditory stimuli was presented to determine characteristic frequency and thresholds. This paradigm consisted of $50 \mathrm{~ms}$-long stimuli (2 presentations per second) with frequencies from $1 \mathrm{kHz}$ to $32 \mathrm{kHz}$ in steps of 1 octave (pseudo-randomly presented), and intensities from $100 \mathrm{~dB}$ to $10 \mathrm{~dB}$ in steps of $10 \mathrm{~dB}$. Next, 100 repeats of $200 \mathrm{~ms}$ and $95 \mathrm{~dB}$ SPL 
BBN stimuli with 2 presentations per second were presented in order to determine response type. Lastly, spontaneous firing was recorded for 5 minutes. Per animal, multiple trajectories were performed. Unstable neurons, and neurons lost during recording, were excluded from further analyses.

\section{Local Field Potentials and Electroencephalography}

After removal of the glass microelectrode, a bipolar electrode was introduced in the MGB (AP -5.7 mm, ML $3.9 \mathrm{~mm}$, and DV $-6 \mathrm{~mm}$ relative to Bregma). This coaxial gold-coated electrode had a platinum-iridium inner wire, shaft diameter of $250 \mu \mathrm{m}$ and tip diameter of approximately $50 \mu \mathrm{m} .{ }^{23}$ Additionally, a cortical electrode consisted of a miniature screw at the vertex, with subcutaneous reference wire, placed on the left mastoid bone. Electrodes were connected to a data acquisition system (PowerLab 8/35, New South Wales, Australia). Signals were sampled at $20.000 \mathrm{~Hz}$ and recorded using LabChart Pro 7 software (ADInstruments, Castle Hill, Australia), high pass filter was set at $0.1 \mathrm{~Hz}$ and low pass filter at $1 \mathrm{kHz}$. Two periods of 5 minutes of data were recorded before and directly after DBS.

\section{Deep brain stimulation}

High frequency stimulation was applied between LFP measurements. Therefore, the bipolar electrode was connected to a constant-current isolator (DLS 100; WPI, Berlin, Germany), which was connected to a stimulator (DS8000; World Precision Instruments, Sarasota, USA). Subjects were stimulated for 5 minutes continuously. Stimulation parameters were based on previous experiments ${ }^{19}$ : frequency of $100 \mathrm{~Hz}, 60 \mu$ s pulse width and $100 \mu \mathrm{A}$ amplitude. Monophasic pulses were applied.

\section{Tissue collection and immunohistochemistry}

At the end of electrophysiological recordings, rats were decapitated and brains were quickly removed and frozen in $-40^{\circ} \mathrm{C}$ 2-methyl-butane (isopentane). Brains were serially cut with a cryostat (Leica CM3050S, Nussloch, Germany) in $50 \mu \mathrm{m}$ thick sections. Standard hematoxylin-eosin staining was performed to evaluate the exact location of tips of stimulation and LFP recording electrodes. 


\section{Data analyses}

Matlab software (V2015A; Mathworks inc., Natick, USA) with custom-written codes was used. Only recordings that showed stable firing were included for further analysis. Spike thresholds were manually selected per dataset by visual and auditory inspection. Spike waveforms were sorted using principal component analysis and $\mathrm{K}$ means clustering, to determine one or multiple single units per recording. Clusters representing single units were selected and confirmed by inspection of their autocorrelation.

All sorted single units were automatically divided into four main response types, based on the PSTH in response to BBN stimuli. Criteria for this categorization were based on previous literature. ${ }^{24,25}$ A neuron was considered a "fast" response type if two criteria were met: 1 ) the response rate within $50 \mathrm{~ms}$ after the stimulus onset was more than 2 standard deviations higher than its spontaneous firing rate, and 2) the response within $50 \mathrm{~ms}$ after stimulus onset rate was more than $30 \%$ higher than the firing rate $50-100 \mathrm{~ms}$ after stimulus onset. If only the first criterion was met, the neuron was considered a "sustained" response type. In case the response rate within 100 ms after stimulus onset was at least 1 standard deviation lower than the spontaneous firing rate, it was considered a "suppressed" neuron. All other units that did not show a response on BBN stimuli were assigned to the "no response" group.

In order to quantify spike irregularity, coefficient of variation (CV) was calculated per unit by dividing the standard deviation of inter spike intervals by the mean. Best frequency of each unit was defined as the frequency that corresponding to the highest firing rate within $50 \mathrm{~ms}$ after stimulus onset for the highest two intensities (90-100 dB). For suppressed response types we took the lowest firing rate (i.e. the strongest suppression). In case two or more stimulus conditions had the same response, we took the best frequency as the average of these frequencies. Response rate heatmaps per stimulus intensity were computed for the best frequency of each unit. Response rate-level functions were calculated for each neuron's best frequency, $16 \mathrm{kHz}$ (the frequency used for noise trauma) and $1 \mathrm{kHz}$ stimulus (far from the affected frequency). To determine $50 \%$ thresholds (L50), a Naka-Rushton function ${ }^{26,27}$ was fitted to the mean response at each stimulus level. The form of the fitted function was: 
$Y=\operatorname{Rmax} x \frac{X^{n}}{L 50^{n}+X^{n}}+R \min$

(Equation 1)

where ' $Y$ ' is the predicted response to a stimulus of level ' $X$ ', 'Rmax' is the maximum response, and ' $\mathrm{L}_{50}$ ' is the stimulus intensity which produces a response equal to $50 \%$ of the difference between ' $R_{\max }$ ' and the spontaneous activity ' $R_{\min }$ ', which we took to be the $50 \%$ threshold. The exponent ' $n$ ' is proportional to the slope of the curve at the intensity $\mathrm{L}_{50}$, for ease of interpretation this was transformed to units of spikes/sec/DB. The four free parameters, 'Rmax', 'L50', 'R $\mathrm{R}_{\min }$ ' and ' $\mathrm{n}$ ' were optimized by minimizing the summed squared error using Matab's Fminsearch function. In addition, we calculated a statistical threshold Lth as level where the fitted firing rate was one SD above the minimum rate $R_{\min }{ }^{28}$ Response dynamic range was calculated as the difference between Lth and the saturating level Lsat, where the fitted rate is one SD below $R_{\max }{ }^{29}$

Fit quality was assessed be the percentage of variance accounted for by the fitted model [30] calculated as:

$$
\% \text { Variance }=100 \times\left(\begin{array}{c}
D(m, r) \\
D R, r)
\end{array}\right)
$$

where ' $\mathrm{D}(\mathrm{m}, \mathrm{r})$ ' corresponds to the mean squared difference between the model predicted response (' $\mathrm{m}$ ', see Equation 1) and the observed mean firing rate (' $\mathrm{r}$ ') at each level, and ' $\mathrm{D}(\mathrm{R}, \mathrm{r})^{\prime}$ corresponds to the mean squared difference between the grand mean firing rate (' $R$ ', calculated across stimulus levels) and mean firing rate at each stimulus, separately.

LFP data were analyzed using the fieldtrip Matlab toolbox ${ }^{31}$ and custom written scripts. To remove line noise, a notch filter was applied at 50, 100 and $150 \mathrm{~Hz}$. Furthermore, for power analysis the data was decomposed using single spectrum decomposition ${ }^{32,33}$ and the component corresponding to $50 \mathrm{~Hz}$ was removed before recombining the data. Data were then down sampled to $250 \mathrm{~Hz}$ and were high-pass filtered above $0.7 \mathrm{~Hz}$. Sections of data containing artifacts were visually identified and rejected. Data were cut into nonoverlapping epochs of 1 second and power and cross-spectrum were calculated using a multitaper method with discrete prolate spheroid sequences (DPSS) for frequencies of 1 to $100 \mathrm{~Hz}$ (smoothing $\pm 4 \mathrm{~Hz}$ ). Connectivity was estimated by calculating debiased weighted phase lag index (dwPLI) from these cross 
spectra. ${ }^{34}$ To test for recovery after DBS, data were separated into 5 bins representing 1-minute time-intervals of the 5 minutes following DBS.

\section{Statistical analyses}

For GPIAS analysis, Wilcoxon Signed Rank test was used and Bonferroni-Holm corrected $\mathrm{p}$ values are presented. Analysis of single units was performed using the following tests. A chi-square test of goodness-of-fit was performed to compare distribution of the four response types between groups. Permutation tests with 1000 randomizations were used to test for differences in spontaneous firing rate (SFR), CV, L50, Rmin, Rmax, slope, Lth and dynamic range between groups. To determine differences in rate-level functions, a mixed ANOVA was performed, including one between-subjects factor "group" (2 levels, noiseexposed or unexposed) and one within-subject factor "stimulus intensity" (10 levels, 10-100 dB). Cluster analysis of spike waveforms obtained from the Gaussian mixture models was performed to test for differences between response types. One-way ANOVA was performed to find a relation between response types and recording coordinates in three dimensions. For LFP analysis, Mixed ANOVA with "group" as between-subjects factor and "time" (6 levels, baseline and 1-5 minutes post DBS) as within-subjects factor were performed to test for differences in phase-synchronization. A significance level alpha for all statistical tests was 0.05. Analysis of behavioral data was performed with SPSS (version 22.0 for Mac, SPSS, Chicago, USA). Matlab Software (R2015a, 64-bit) was used to analyze electrophysiological data.

\section{Results}

\section{Gap-Prepulse Inhibition of Acoustic Startles}

Results of GPIAS are presented in Figure 5.1. Gap:no gap ratios were increased in the noise-exposed group for the 16 and $20 \mathrm{kHz}$ background sound $(Z=2.55$, $p=0.01$ and $Z=2.66, p=0.01$ respectively), but not for $10 \mathrm{kHz}(Z=1.24, p=0.21)$. The control group did not show altered gap:no gap ratios $(Z=-0.14, p=0.89$ for $10 \mathrm{kHz}, \mathrm{Z}=-1.21, p=0.23$ for $16 \mathrm{kHz}$, and $\mathrm{Z}=-1.48, p=0.14$ for $20 \mathrm{kHz}$ ). These GPIAS responses are similar to earlier published studies $19,21,35$ and indicate existence of tinnitus in the noise-exposed group. Our setup with freely moving subjects did not allow to validly discriminate individual tinnitus positive and 
negative animals, therefore we did not exclude subjects and labelled our group as "noise-exposed".
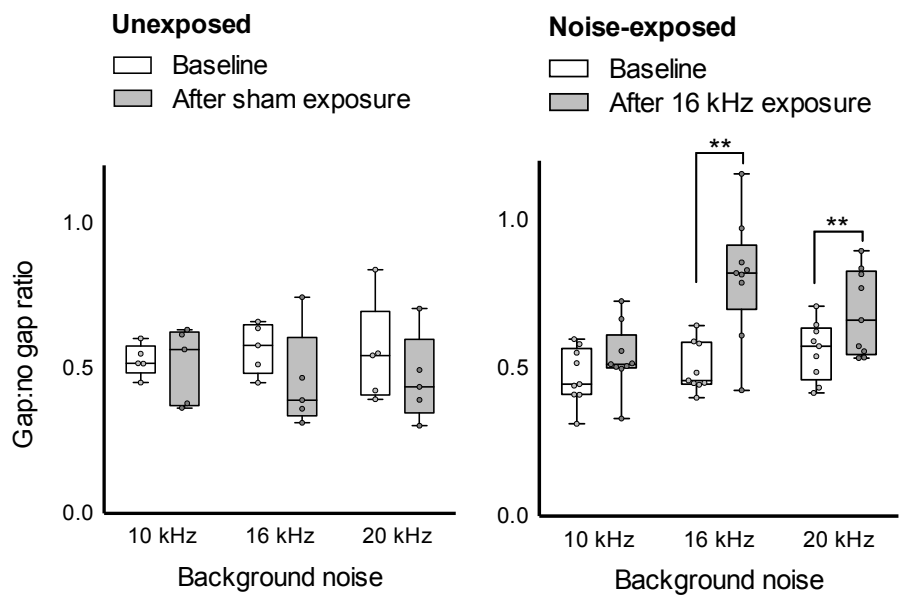

Figure 5.1 Gap:no gap ratios of both study groups. Detection of the gap was significantly reduced after $16 \mathrm{kHz}$ noise exposure for the 16 and $20 \mathrm{kHz}$ background sound, ${ }^{* *} p<0.01$

\section{Single Unit Recordings}

In total, 214 single units were identified ( $\mathrm{n}=145$ (average of 16.1 per subject) in the noise-exposed group, $n=69$ (average of 13.8 per subject) in unexposed control group). Based on PSTH during BBN stimuli, four response types were distinguished (Figure 5.2A): I) fast response ( $\mathrm{n}=28$ for the noise-exposed group and $n=29$ for unexposed controls), II) sustained response $(n=28$, and $n=11,3)$, III) suppressed response $(n=27$, and $n=10)$, and IV) no response $(n=62$, and $\mathrm{n}=19$ ). Most of the units of group IV were found in the middle of a trajectory with adjacent responding neurons, suggesting these responses were likely within the MGB. The proportions of response types differed between the noiseexposed group and the unexposed control group, $\mathrm{X}^{2}(3, \mathrm{~N}=214)=12.68, p=0.005$ (Figure 5.2B). Notably there was a higher proportion of non-responsive units and a lower proportion of fast responsive neurons in the noise-exposed group compared to the control group, whereas the proportions of sustained and suppressed neurons were similar in both groups. 
Characteristics of single unit recordings during 5-minute spontaneous recording are presented in Table 5.1. Overall, there was increased spontaneous firing in the noise-exposed subjects (mean SFR of $9.65 \pm 0.7$ and $7.40 \pm 0.9$, $p=0.03$ ). Subgroup analysis revealed that the suppressed response-type neurons showed a significantly higher spontaneous firing rate in the noise-exposed group (mean of $15.06 \pm 1.4$ spikes/s, compared to $9.68 \pm 2.1$ spikes/s, $p=0.006$. An increase was also observed in the sustained response type, which almost reached significance (mean $9.77 \pm 2.2$ and $4.20 \pm 1.9$ spikes/s, $p=0.052$ ). In the fast-responding and no-responding neurons, there were no differences. There was no significant difference in CV between the two groups.

A
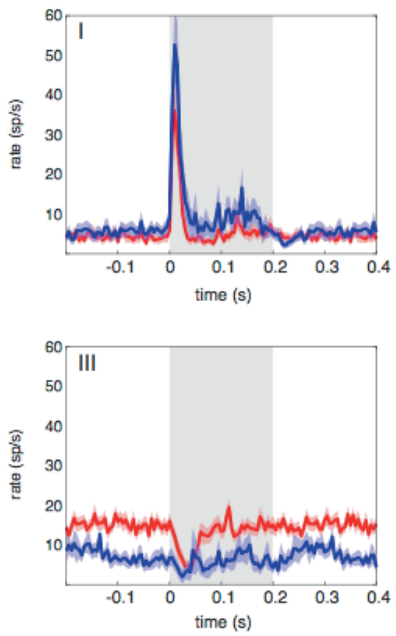
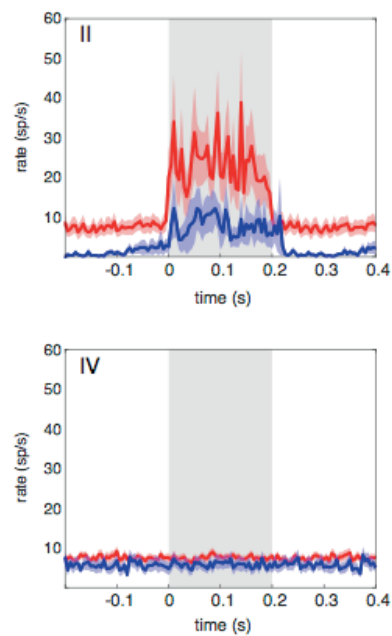

B
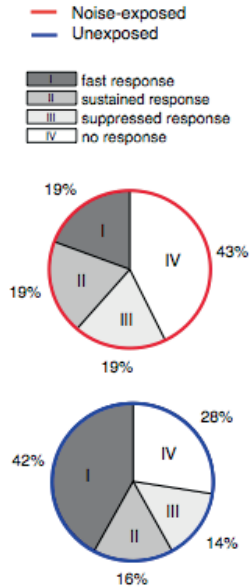

Figure 5.2 A) PSTH of all single unit recordings during BBN stimuli, divided in four response types: I) fast response, II) sustained response, III) suppressed response, and IV) no response. Solid lines show mean response, shading shows \pm SEM. B) Proportions of response types differed per study group. $n=145$ in noise-exposed subjects, $n=69$ in unexposed controls. 
The sensitivity of individual neurons was characterized by fitting a NakaRushton function ${ }^{26,27}$ to response rates at 10 sound intensities. Here we considered the units where the fit quality exceeded an explained variance of $70 \%$. Physiological parameters of the rate-level functions are presented in Table 5.2. Thresholds ( $\mathrm{L}_{50}$ and $\mathrm{Lth}$ ) of fast responding units were significantly higher, indicating lower sensitivity, in the noise-exposed group. The other response types did not show altered L50 or Lth thresholds. The other parameters, Rmin, $R_{\max }$ and slope and dynamic range, did not statistically differ between groups.

Surface plots and rate-level functions during frequency tuning measurements are shown per response type in Figure 5.3. Visual inspection of the surface plots (Figure 5.3A) reveals two main differences between the noise-exposed and unexposed control group. First, spontaneous firing of the sustained and suppressed neurons was higher in the noise-exposed group, in line with results from Table 5.1. Second, for fast responding neurons, stimulus evoked responses were higher particularly for lower intensities in the unexposed group. This is in line with results from Table 5.2, where the thresholds were higher in the noise-exposed group for this neuron type. For units with a sustained response the pattern was more mixed.

To summarize the surface plots, we calculated rate-level functions from firing rate during $0.05 \mathrm{~s}$ after stimulus onset. Analysis of the rate-level functions of fast responding cells during presentation of the best frequency (Figure 5.3B, first column) revealed a significant interaction between group (noise-exposed or unexposed control) and stimulus intensity $(\mathrm{F}(1,9)=3.23, p<0.001)$. In unexposed controls, higher stimulus intensities led to higher firing rate compared to the noise-exposed group (main group effect $\mathrm{F}(1,55)=6.74, p=0.01$ ). Interestingly, for the sustained cells, there were no significant differences between groups. The suppressed response type showed a difference in firing rate between groups regardless of stimulus intensity (main group effect $\mathrm{F}(1,35)=4.83, p=0.03)$. Analysis of responses to the $16 \mathrm{kHz}$ noise-trauma frequency Figure 5.3B, second column) revealed different results. In both fast and sustained responding neurons, no effect of noise-trauma was found. Notably, there was a significant group difference for the suppressed neurons $(\mathrm{F}(1,35)=4.54, p=0.04)$, which was independent of intensity of the stimuli. For the $1 \mathrm{kHz}$ control frequencies, there were no significant group differences found for any response type. 

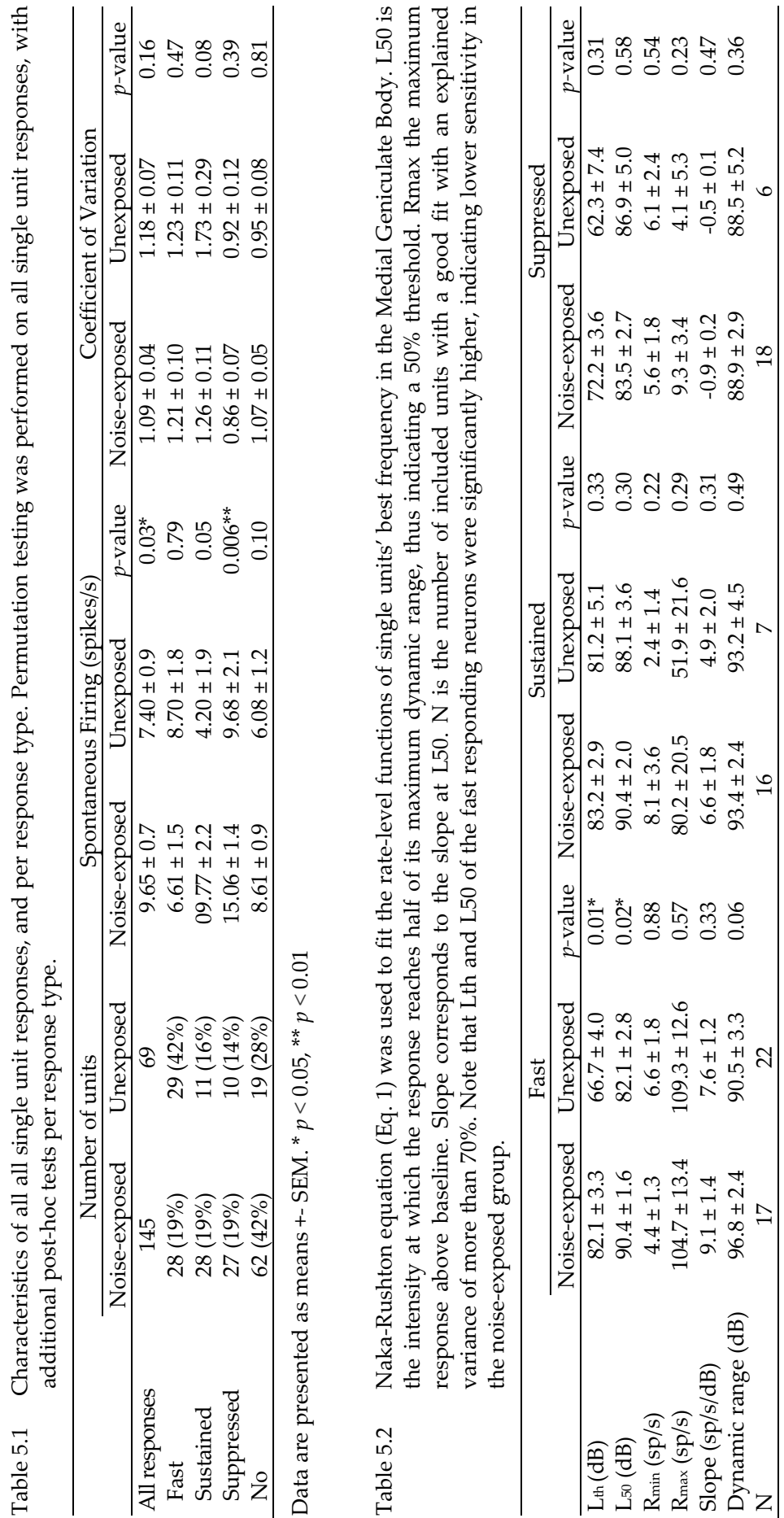
$\mathbf{A}$

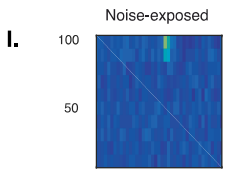

II.

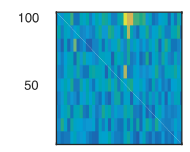

III.

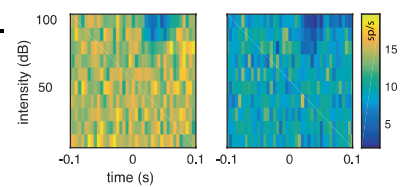

B
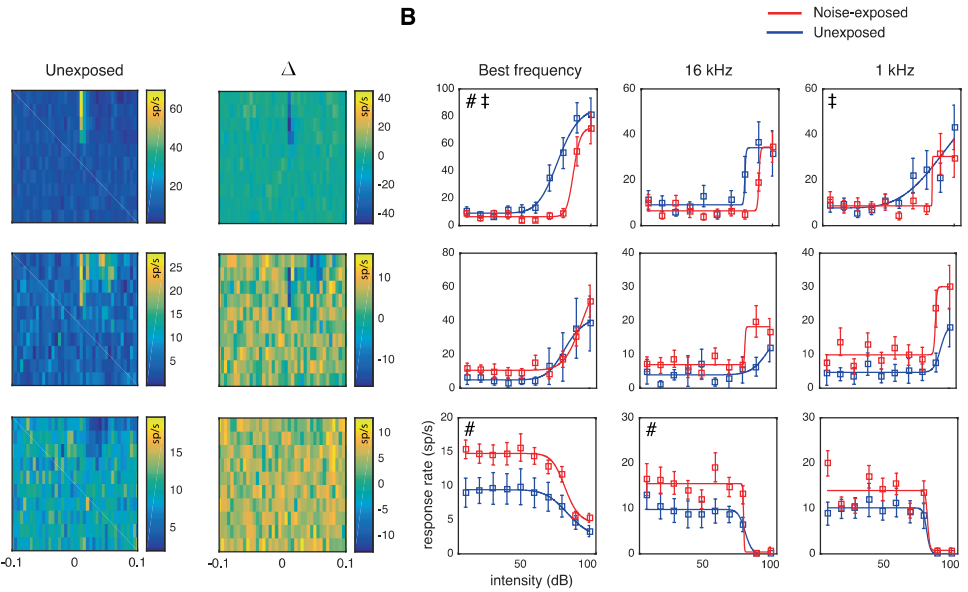

Figure 5.3 A) Mean firing rate heatmaps for the best frequency per group and per response type: I) fast response, II) sustained response, III) suppressed response. B) Rate-level functions per response type for the best frequency (first column), $16 \mathrm{kHz}$ (second column) and $1 \mathrm{kHz}$ (third column) stimuli. Notice that the noise-exposed group shows a lower response rate in fast responding neurons, but not in sustained neurons. The group difference in the suppressed neurons was only significant for responses to the best frequency and the $16 \mathrm{kHz}$ tinnitus frequency. This can be related to the increased spontaneous firing in the noise-exposed group. Means \pm SEM are presented. Solid lines show Naka-Ruston fit to population means. \# indicates significant group effect (noise-exposed vs. unexposed control). $\ddagger$ indicates significant interaction (group * intensity).

\section{Local Field Potentials and Electroencephalography}

\section{a. Electrode localization}

After recording single unit activity, the microelectrode was replaced with a DBS probe capable of recording LFP and providing electrical stimulation. The DBS electrode positions used for LFP recording and high frequency stimulation are visualized in Figure 5.4. 


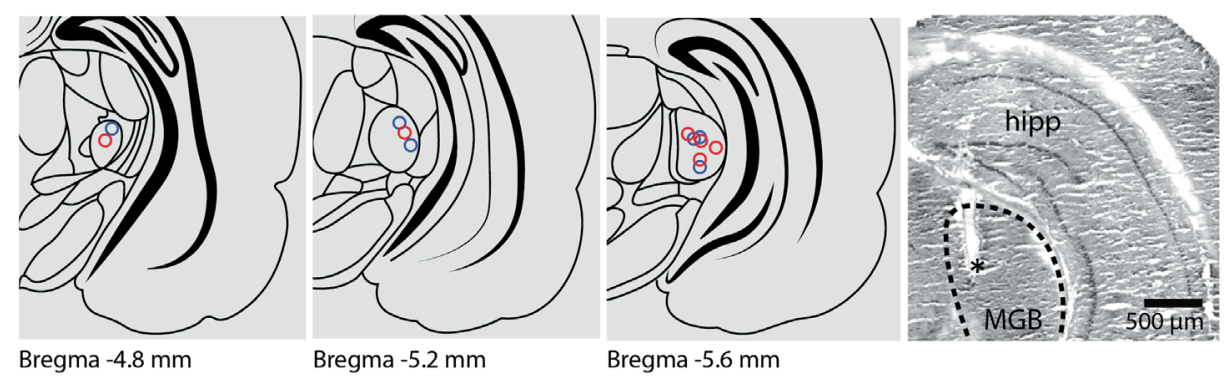

Figure 5.4 Schematic representation of all LFP electrode locations in the MGB. On the right a representative example of $\mathrm{H} \& \mathrm{E}$ stained section with visible electrode trajectory. Two subjects (electrode locations not in figure) were excluded from LFP analysis due to incorrect positioning and hardware failure. Noise-exposed in red, unexposed control in blue. ${ }^{*}$ Marks the location of the electrode tip. MGB, medial geniculate body. Hipp, hippocampus.

\section{b. Phase-synchronization and power}

Phase-synchronization between MGB and cortex and power were analyzed at baseline (5 minutes) and in 1-minute time-intervals after high-frequency stimulation of the MGB (Figure 5.5A). Statistical analysis of thalamocortical phase-synchronization revealed significant main effects of DBS (time) at the beta frequency band $(20-35 \mathrm{~Hz}, \mathrm{~F}(1,5)=4.90, p<0.001)$, and gamma frequency $(50-70 \mathrm{~Hz}, \mathrm{~F}(1,5)=3.02, p=0.02)$. All effects were independent of group, as there were no significant interactions. For delta $(1-4 \mathrm{~Hz})$, theta $(4-8 \mathrm{~Hz})$, and alpha $(8-12 \mathrm{~Hz})$ band, no significant interactions or main effects were observed. Analysis of power spectra showed a significant suppressing effect of DBS on all tested frequency bands, without a group effect (Figure 5.5B) in the MGB electrode. For all frequency bands, DBS led to a brief increase in power, after which it decreased to levels lower than baseline (delta $F(1,5)=8.89, p<0.001$, theta $\mathrm{F}(1,5)=7.42, p<0.001$, alpha $\mathrm{F}(1,5)=6.24, p<0.001$, beta $\mathrm{F}(1,5)=3.02, p=0.02$, and gamma $F(1,5)=3.67, p=0.01$. At the cortical level, power at these frequency bands did not significantly change after application of DBS. 
A

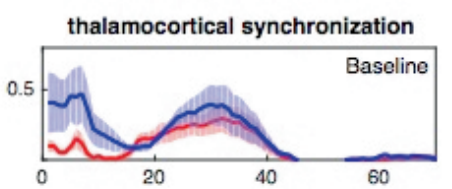

5 minutes DBS
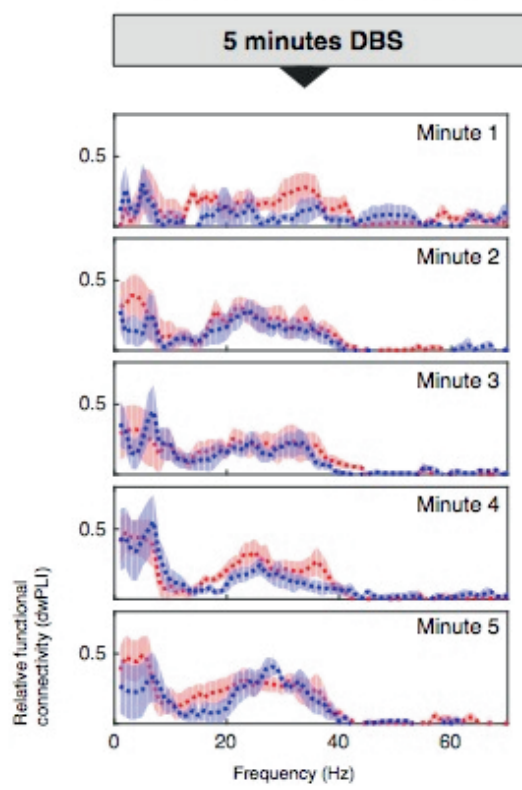

B

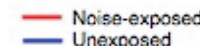

Unexposed

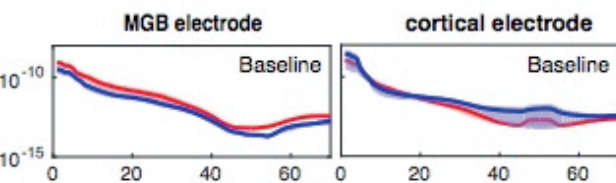

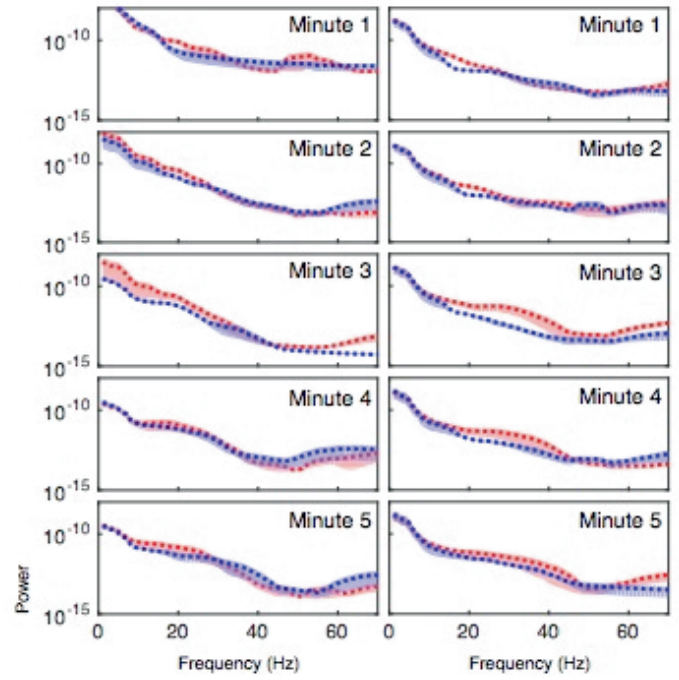

Figure 5.5 LFP recordings at baseline before MGB DBS $(100 \mathrm{~Hz}, 60 \mu \mathrm{s}, 100 \mu \mathrm{A})$, and 5 separate minutes directly after 5 minutes of MGB DBS. A) Phase-synchronization between MGB and cortex (debiased weighted phase lag index (dwPLI)) of both study groups. DBS caused a significant reduction in relative functional connectivity in the beta (20-35 Hz) and gamma $(50-70 \mathrm{~Hz})$ frequency band which diminished over time. B) Mean power spectra recorded by MGB and cortical electrodes. There was a significant suppressing effect of DBS on power in all frequency bands in both channels.

\section{Discussion}

In this study, we examined neurophysiological characteristics of the MGB and the effects of DBS of the MGB in the noise-exposed animal model. Our main findings are first, a reduction in the population of fast responding neurons and a corresponding increase in non-responsive neurons after noise-exposure. Second, acoustic over-exposure lead to an increased spontaneous firing of 
sustained and suppressed response type neurons without influencing the responsiveness to stimuli. Third, fast responding neurons in noise-exposed subjects showed an increased threshold without a change in the spontaneous firing rate. Additionally, we found that MGB DBS suppressed thalamocortical synchronicity in both noise-exposed and control animals.

\section{Neurophysiological hallmarks of the MGB in the noise- exposed tinnitus animal model}

Spontaneous firing rates were increased in the noise-exposed group. This finding was in line with reports by others in awake, ${ }^{16}$ but not in anesthetized ${ }^{17}$ tinnitus animals. While previous reports did not differentiate between neuron types, our results show that this increase in spontaneous firing was only present in a subtype of MGB neurons: only the suppressed and sustained response type neurons but not fast and non-responding neurons. Interestingly, in the noise-exposed animals the number of fast responding neurons was reduced, while there was an increase in the proportion of non-responding neurons. The proportion of sustained and suppressed responding neurons remained the same. The question arises of whether specific response types belong to different excitatory or inhibitory neuronal cell types. We could not find differences in waveform, nor location of recording (Figure S5.1 and S5.2 in supplemental material). In the rat MGB, there are morphologically different cell types. ${ }^{36}$ GABAergic neurons are in low abundance, likely less than $1 \%$ in the MGB of the rat, ${ }^{37}$ and most recorded neurons are probably glutamatergic. The increased spontaneous activity of sustained and suppressed response types might therefore reflect an increase in excitatory neuronal activity. Hyperactivity of sustained and suppressed neurons might be the readout at a single neuron level of thalamocortical dysrhythmia as described by other electrophysiological studies at the population and network level.,38

The undisputed connection between tinnitus, hearing loss and hyperacusis is a major challenge in the field of tinnitus research. ${ }^{1}$ These symptoms often coexist and share similar etiological and pathophysiological factors. It has recently been postulated that there is a certain degree of hearing loss in almost all human and animal tinnitus cases, which is sometimes undetectable by audiograms or ABR. ${ }^{39,40}$ A limitation of our study is that we only compared noise-exposed animals to sham exposed controls. Our results confirmed the existence of tinnitus-like behavior on a group level, but our setup did not allow 
to validly differentiate tinnitus positive or negative animals on an individual level. Therefore, it remains uncertain what the confounding effects of these two symptoms are in our findings.

Based on our findings, it might be postulated that different characteristics of response types reflect different symptoms. We propose that tinnitus perception might be due to increased spontaneous activity of sustained and suppressed neurons, possibly contributing to increased oscillations and thalamocortical dysrhythmia. Hearing loss could be reflected by the increase in the proportion of non-responding neurons, together with the loss of fast response type neurons and their increased thresholds. Hyperacusis might be related to an increased response to external stimuli, which has been shown before. ${ }^{16}$ In our study, we found an increased response in sustained neurons, but this difference did not reach significance between groups. These hypotheses definitely need further validation. It is tempting to speculate that loss of fastresponding neurons is causally linked to the increase in spontaneous firing rate of sustained and suppressed neurons. We however did not find a correlation between the two factors, which might be due to the small sample size. Additional electrophysiological studies in both animal models and humans would be valuable and might relate specific properties of the MGB neural subtypes to hearing loss, hyperacusis and tinnitus.

Thalamocortical dysrhythmia has been proposed as an underlying mechanism of tinnitus.,11 In our study, no differences in power and corticothalamic synchronization between noise-exposed tinnitus animals and controls were found. Thalamocortical dysrhythmia and synchronization by loss of inhibition has been shown in the salicylate induced tinnitus model. An increased cortical gamma activity was proven to be associated with enhanced theta-gamma coupling, as well as a decreased alpha power and coherence between the auditory cortex and the MGB. ${ }^{38}$ Our negative finding might be due to a small sample size, as our study was not powered to find group effects for LFP recordings. In order to avoid type 2 error, firm conclusions cannot be drawn on the effect of noise-trauma on thalamocortical synchronization based on our study. 


\section{Deep brain stimulation of the MGB}

Previously, it has been shown that MGB DBS suppresses tinnitus-like behavior in rats. ${ }^{19}$ The current study shows a temporary disrupting effect of MGB DBS on corticothalamic synchronization in the beta and gamma band. Furthermore, we found a suppressing effect on the total power of the LFP in all frequency bands after MGB DBS.

The exact working mechanism of DBS remains elusive. An accepted principle is that high frequency DBS causes an inactivation or a temporary lesion of the target area. ${ }^{41}$ In the noise-exposed animal model as used in the current study, spontaneous hyperactivity within the MGB could well be related to tinnitus. MGB DBS might suppress this aberrant activity. Other studies report a downregulation of inhibitory neurotransmission in the inferior colliculus in tinnitus. ${ }^{42-44}$ High frequency DBS at the MGB could cause presynaptic GABA release of these projections and herewith suppress MGB activity. Furthermore, stimulation might suppress cell body activity by inducing a depolarization block. Besides a local inhibitory effect, DBS also causes distant excitatory actions. Direct stimulation of axons nearby the electrode can induce tonic patterns of action potential in structures that are in a distance from the stimulated target. Firing characteristics of postsynaptic cells might be influenced by multiple underlying effects, such as effects on neurotransmitter release, changes in metabolic activity and plastic changes such as long-term potentiation and depression. ${ }^{41}$ Considering these complex distant effects, activation of reticular nucleus neurons by MGB DBS might influence thalamic gating function in tinnitus. Overall, above-mentioned mechanisms might lead to increased thalamocortical gamma oscillations, which have been associated with tinnitus. ${ }^{45}$ Our findings support the theory that MGB DBS blocks abnormal information flow, such as thalamocortical dysrhythmia, as we found a desynchronizing effect at the higher frequencies after MGB DBS.

In line with our previous behavioral study, ${ }^{19}$ the effect of MGB DBS on synchronization was residual, as corticothalamic synchronization in the beta and gamma bands was initially suppressed, but returned approximately to baseline levels within 5 minutes. MGB DBS can thus have neurophysiological effects that extend the stimulation period and may induce neural plasticity. This finding is important for future clinical application of MGB DBS in humans. Stimulation could be performed in ON and OFF cycles, resulting in a 
longer battery life, reduction of stimulation induced side-effects and habituation effects.

\section{Conclusion}

The MGB has a prominent role in auditory pathophysiology such as tinnitus. Based on single unit recordings, we propose a distinct role for separate functional classes of neurons affected by noise trauma, whereby fastresponding neurons become less- or non-responsive without change to their spontaneous rate, while sustained and suppressed type neurons exhibit enhanced spontaneous activity without change to their stimulus driven activity. It seems plausible that these findings are strongly correlated to tinnitus. In this light, our LFP findings support suppression of thalamocortical synchronization as an underlying mechanism of tinnitus suppression by high frequency MGB DBS. Further electrophysiological studies are needed to investigate the role of different neural response types in neuropathophysiology of auditory trauma, as well as the possible effects of MGB DBS on thalamocortical dysrhythmia and thalamic gating function. 


\section{References}

1. Shore SE, Wu C. Mechanisms of Noise-Induced Tinnitus: Insights from Cellular Studies. Neuron. 2019;103(1):8-20.

2. Rauschecker JP, Leaver AM, Muhlau M. Tuning out the noise: limbic-auditory interactions in tinnitus. Neuron. 2010;66(6):819-826.

3. De Ridder D, et al. Thalamocortical Dysrhythmia: A Theoretical Update in Tinnitus. Front Neurol. 2015;6:124.

4. Eggermont JJ, Roberts LE. The neuroscience of tinnitus. Trends Neurosci. 2004;27(11):676-682.

5. Roberts LE, et al. Ringing ears: the neuroscience of tinnitus. J Neurosci. 2010;30(45): 14972-14979.

6. Kaltenbach JA. Tinnitus: Models and mechanisms. Hear Res. 2011;276(1-2):52-60.

7. Caspary DM, Llano DA. Auditory thalamic circuits and GABAA receptor function: Putative mechanisms in tinnitus pathology. Hear Res. 2017;349:197-207.

8. Leaver AM, et al. Dysregulation of limbic and auditory networks in tinnitus. Neuron. 2011; 69(1):33-43.

9. Winer JA, et al. Origins of medial geniculate body projections to physiologically defined zones of rat primary auditory cortex. Hear Res. 1999;130(1-2):42-61.

10. Bartlett EL. The organization and physiology of the auditory thalamus and its role in processing acoustic features important for speech perception. Brain Lang. 2013;126(1):29-48.

11. Llinas RR, et al. Thalamocortical dysrhythmia: A neurological and neuropsychiatric syndrome characterized by magnetoencephalography. Proc Natl Acad Sci U S A. 1999;96(26): $15222-15227$.

12. Lanting $\mathrm{CP}$, de Kleine $\mathrm{E}$, van Dijk $\mathrm{P}$. Neural activity underlying tinnitus generation: results from PET and fMRI. Hear Res. 2009;255(1-2):1-13.

13. Muhlau M, et al. Structural brain changes in tinnitus. Cereb Cortex. 2006;16(9):1283-1288.

14. Landgrebe $M$, et al. Structural brain changes in tinnitus: grey matter decrease in auditory and non-auditory brain areas. Neuroimage. 2009;46(1):213-218.

15. Smits M, et al. Lateralization of functional magnetic resonance imaging (fMRI) activation in the auditory pathway of patients with lateralized tinnitus. Neuroradiology. 2007;49(8): 669-679.

16. Kalappa BI, et al. Single unit hyperactivity and bursting in the auditory thalamus of awake rats directly correlates with behavioural evidence of tinnitus. J Physiol. 2014;592(22): 5065-5078.

17. Barry KM, Robertson D, Mulders W. Changes in auditory thalamus neural firing patterns after acoustic trauma in rats. Hear Res. 2019;379:89-97.

18. Basta D, Goetze R, Ernst A. Effects of salicylate application on the spontaneous activity in brain slices of the mouse cochlear nucleus, medial geniculate body and primary auditory cortex. Hear Res. 2008;240(1-2):42-51.

19. van Zwieten G, et al. Inhibition of Experimental Tinnitus With High Frequency Stimulation of the Rat Medial Geniculate Body. Neuromodulation. 2019;22(4):416-424.

20. van Zwieten G, et al. Tinnitus: Is there a place for brain stimulation? Surg Neurol Int. 2016; 7(Suppl 4):S125-129.

21. Smit JV, et al. Deep brain stimulation of the inferior colliculus in the rodent suppresses tinnitus. Brain Res. 2016;1650:118-124.

22. Paxinos GW, C. The rat brain in stereotaxic coordinates: hard cover edition. 2006, New York: Academic press.

23. Tan S, et al. Experimental deep brain stimulation in animal models. Neurosurgery. 2010; 67(4):1073-1079; discussion1080.

24. Bordi F, LeDoux JE. Response properties of single units in areas of rat auditory thalamus that project to the amygdala. II. Cells receiving convergent auditory and somatosensory inputs 
and cells antidromically activated by amygdala stimulation. Exp Brain Res. 1994;98(2): 275-286.

25. Calford MB, Webster ER. Auditory representation within principal division of cat medial geniculate body: an electrophysiology study. J Neurophysiol. 1981;45(6):1013-1028.

26. Naka KI, Rushton WA. S-potentials from colour units in the retina of fish (Cyprinidae). J Physiol. 1966;185(3):536-555.

27. Albrecht DG, Hamilton DB. Striate cortex of monkey and cat: contrast response function. J Neurophysiol. 1982;48(1):217-237.

28. Wen $\mathrm{B}$, et al. Dynamic range adaptation to sound level statistics in the auditory nerve. J Neurosci. 2009;29(44):13797-13808.

29. Watkins PV, Barbour DL. Level-tuned neurons in primary auditory cortex adapt differently to loud versus soft sounds. Cereb Cortex. 2011;21(1):178-190.

30. Carandini M, Heeger DJ, Movshon JA. Linearity and normalization in simple cells of the macaque primary visual cortex. J Neurosci. 1997;17(21):8621-8644.

31. Oostenveld R, et al. FieldTrip: Open source software for advanced analysis of MEG, EEG, and invasive electrophysiological data. Comput Intell Neurosci. 2011;2011:156869.

32. Bonizzi $P$, et al. Singular spectrum analysis improves analysis of local field potentials from macaque V1 in active fixation task. Conf Proc IEEE Eng Med Biol Soc. 2012;2012:2945-2948.

33. Bonizzi P, et al. Singular Spectrum Decomposition: A New Method For Time Series Decomposition. Advances in Adaptive Data Analysis. 2014;06(04).

34. Vinck M, et al. An improved index of phase-synchronization for electrophysiological data in the presence of volume-conduction, noise and sample-size bias. Neuroimage. 2011;55(4): 1548-1565.

35. van Zwieten G, et al. Alleviation of Tinnitus With High-Frequency Stimulation of the Dorsal Cochlear Nucleus: A Rodent Study. Trends Hear. 2019;23:2331216519835080.

36. Clerici WJ, Coleman JR. Anatomy of the rat medial geniculate body: I. Cytoarchitecture, myeloarchitecture, and neocortical connectivity. J Comp Neurol. 1990;297(1):14-31.

37. Winer JA, Larue DT. Evolution of GABAergic circuitry in the mammalian medial geniculate body. Proc Natl Acad Sci U S A. 1996;93(7):3083-3087.

38. Vianney-Rodrigues P, Auerbach BD, Salvi R. Aberrant thalamocortical coherence in an animal model of tinnitus. J Neurophysiol. 2019;121(3):893-907.

39. Weisz N, et al. High-frequency tinnitus without hearing loss does not mean absence of deafferentation. Hear Res. 2006;222(1-2):108-114.

40. Kujawa SG, Liberman MC. Adding insult to injury: cochlear nerve degeneration after "temporary" noise-induced hearing loss. J Neurosci. 2009;29(45):14077-14085.

41. Hamani C, Temel Y. Deep brain stimulation for psychiatric disease: contributions and validity of animal models. Sci Transl Med. 2012;4(142):142rv8.

42. Bauer CA, et al. Tinnitus and inferior colliculus activity in chinchillas related to three distinct patterns of cochlear trauma. J Neurosci Res. 2008;86(11):2564-2578.

43. Dong $\mathrm{S}$, et al. Changes in neuronal activity and gene expression in guinea-pig auditory brainstem after unilateral partial hearing loss. Neuroscience. 2009;159(3):1164-1174.

44. Sametsky EA, et al. Enhanced GABAA-Mediated Tonic Inhibition in Auditory Thalamus of Rats with Behavioral Evidence of Tinnitus. J Neurosci. 2015;35(25):9369-9380.

45. Llinas R, et al. Rhythmic and dysrhythmic thalamocortical dynamics: GABA systems and the edge effect. Trends Neurosci. 2005;28(6):325-333. 


\section{Supplemental material}

Results presented so far have shown a clear effect of noise exposure on the distribution of the four response types. We questioned whether these differences might be ascribed to cell type or differences in location within the MGB. Inspection of the average spike waveform of the four groups and cluster analysis obtained from the Gaussian mixture model could not distinguish differences in waveform shape (Figure S5.1). Furthermore, one-way ANOVA analysis revealed no significant relationship between the recording coordinates in three dimensions and response type, suggesting no effect of recording location within the MGB (Figure S5.2).
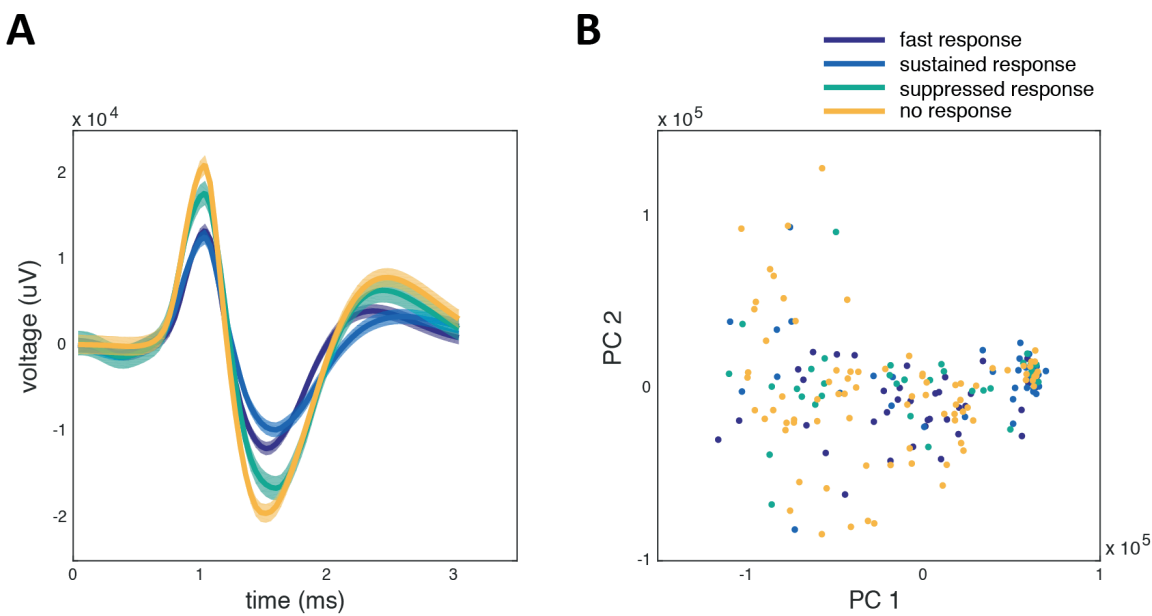

Figure S5.1 A) Waveforms per response type. Solid lines show mean response, shading shows \pm SEM. B) Clusters of spike waveforms obtained from the Gaussian mixture model. Colors represent four different response types. 


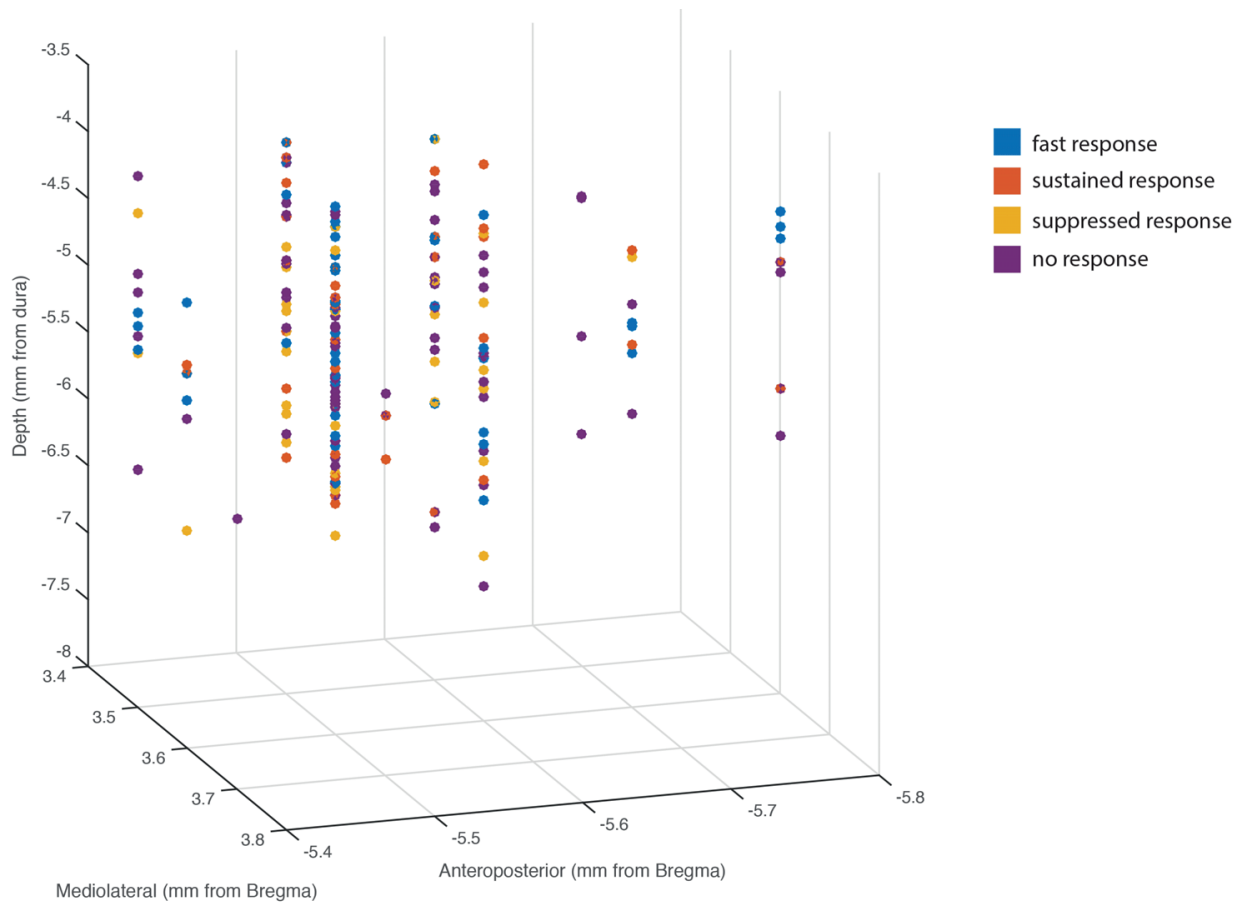

Figure S5.2 Overview of stereotactic coordinates of all recorded single units. All four response types are evenly distributed among the three axes and intermixed along recording trajectories. 



\title{
The effect of noise trauma and high frequency stimulation on thalamic sensory gating in rodents
}

\author{
Van Zwieten $G^{*}$ \\ Zare A* \\ Kotz SA \\ Temel Y \\ Schultz BG \\ Schwartze M* \\ Janssen MLF * \\ * First and last authorships are shared
}




\section{Abstract}

Background: The medial geniculate body (MGB) of the thalamus plays a central role in tinnitus pathophysiology. Breakdown of sensory gating in this part of the auditory thalamus is a potential mechanism underlying tinnitus. The alleviation of tinnitus-like behavior by high frequency stimulation (HFS) of the MGB might mitigate dysfunctional sensory gating.

Objective: The study aims at exploring the role of the MGB in sensory gating as a mandatory relay area in auditory processing in noise-exposed and control subjects, and to assess the effect of MGB HFS on this function.

Methods: Noise-exposed rats and controls were tested. Continuous auditory sequences were presented to allow assessment of sensory gating effects associated with pitch, binary grouping, and temporal regularity. Evoked potentials (EP) were recorded from the MGB and acquired before and after HFS $(100 \mathrm{~Hz})$.

Results: Noise-exposed rats showed differential modulation of MGB EP amplitudes, confirmed by significant main effects of stimulus type, pair position and temporal regularity. Noise-exposure selectively abolished the effect of temporal regularity on EP amplitudes. A significant three-way interaction between HFS phase, temporal regularity and rat condition (noiseexposed, control) revealed that only noise-exposed rats showed significantly reduced EP amplitudes following MGB HFS.

Conclusion: This is the first report that shows thalamic filtering of incoming auditory signals based on different sound features. Noise-exposed rats further showed higher EP amplitudes in most conditions and did not differentiate the temporal regularity. Critically, MGB HFS was effective in reducing amplitudes of the EP responses in noise-exposed animals. 


\section{Introduction}

Tinnitus sufferers perceive a sound in the absence of an external audible sound source. Patients suffering from tinnitus often experience a substantially lower quality of life and effective therapies are still lacking. A limited understanding of tinnitus pathophysiology hinders the development of effective treatments. Although the specific pathophysiological mechanisms underlying tinnitus remain elusive, multiple theories with overlapping neurophysiological mechanisms have been proposed. The central gain model proposes that tinnitus results from dynamic compensatory gain enhancement. ${ }^{1}$ Accordingly, there is an increase of evoked neural responses following noise trauma. ${ }^{2}$ More specifically, increased 'gain' in auditory neurons would lead to alterations in synaptic transmission as well as neuronal excitability and synchrony. ${ }^{2,3}$ Furthermore, breakdown of sensory gating (SG) has been proposed as a mechanism underlying phantom auditory perception. ${ }^{4,5}$ All mechanisms might contribute to altered oscillations within the thalamocortical loop, described as thalamocortical dysrhythmia. ${ }^{6}$

SG is defined as the adaptive filtering of changing stimulus features (gating in) relative to repetitive stimulus features (gating out). 4,5 According to this model, the tinnitus sensation is linked to the failure of sensory-perceptual filtering or "noise cancelling system". The medial geniculate body (MGB) of the thalamus is a major relay between midbrain and cortex, and acts as the gatekeeper for auditory signals. Therefore, we expected that the MGB plays an important role in dysfunctional SG in tinnitus. How this thalamic gate functions and how it can potentially be modulated, remains poorly understood. ${ }^{7}$ It has been hypothesized that the MGB is mainly inhibited by the thalamic reticular nucleus, a structure that derives input from a number of paralimbic structures. ${ }^{8}$ This inhibitory feedback loop may block irrelevant sensory inputs and this mechanism might be altered in tinnitus. To our knowledge, only cortical features of SG in tinnitus have been directly studied so far. ${ }^{9,10}$

SG may operate on the basis of different features of auditory input, including the specific stimulus type and timing, (i.e. sound structure and temporal arrangement in terms of grouping and regularity). Together, these features define the parameters that guide filtering. Such filtering dismisses goalirrelevant neural signals in favor of selective attention to goal-relevant information. ${ }^{11}$ Dysfunctional SG is evident in various neuropsychiatric 
conditions, such as impulsivity control disorders and schizophrenia. ${ }^{12,13}$ Recent evidence points to the possibility of tinnitus as a possible further condition in which impaired SG plays a critical role. Correlations between tinnitus severity and decreased SG via the Pa component of cortical auditory evoked potentials and an increased SG via the N1 component have recently been described.(9) SG is also influenced by state changes of the organism. ${ }^{14}$ For example, a casecontrol study showed that there is a relation between the level of SG and behavioral aspects of tinnitus based on the tinnitus handicap and sensory gating inventories. ${ }^{10}$ However, it is unclear how SG is implemented in thalamic processing stages, and if SG can be modulated in the specific context of tinnitus.

Deep brain stimulation (DBS) is a potential method of exerting a modulatory influence on auditory SG in tinnitus. DBS has recently emerged as a promising treatment option for tinnitus. ${ }^{15-17}$ High frequency stimulation (HFS) applied to the auditory pathway has been shown to effectively reduce tinnitus-like behavior in rats. ${ }^{18-20}$ The exact working mechanisms of DBS remain elusive, although complex inhibitory and distant excitatory effects have been described. ${ }^{15,21}$

In the current study, we tested the hypothesis that SG occurs at the level of the MGB and can be altered in a noise-exposed tinnitus animal model. Considering the positive effect of MGB HFS on tinnitus-like behavior, we hypothesize that HFS of the MGB modulates thalamic SG. In other words, HFS might restore a dysfunctional "noise cancelling system" by counteracting aberrant filtering of sensory input at the level of the MGB. ${ }^{21}$

To this end, we investigated auditory SG in the MGB in control and noiseexposed tinnitus rats and further explored the effect of HFS. Tinnitus was assessed using gap-prepulse inhibition of acoustic startle (GPIAS). EP recordings were conducted in anesthetized animals, using paired-tone auditory stimulus sequences that were composed of different pitches (frequent standard and infrequent deviant tones), position (temporal order within the pair) and temporal regularity (regular [isochronous] and irregular), before and after MGB HFS. 


\section{Materials and methods}

\section{Subjects}

The experimental group used for the current study has been described before..$^{22}$ Briefly, a total of 13 male Sprague Dawley rats were used, divided into two groups: i) noise-exposed tinnitus $(n=8)$ and ii) unexposed controls $(n=5)$. Rats were individually housed in standard Makrolon ${ }^{\mathrm{TM}}$ cages, with food and water ad libitum. Conditions in the room were constant, with a temperature of $20^{\circ} \mathrm{C}$ to $22^{\circ} \mathrm{C}$ and humidity of $60 \%$ to $70 \%$. The light-dark cycle was reversed, and experiments were conducted within the dark period. The study was approved by the Animal Experiments and Ethics Committee at Maastricht University, the Netherlands.

\section{Study design}

The overall design of the study comprised four main parts: GPIAS, noise exposure, repeated GPIAS, and recording of EPs and HFS. The following readout parameters were used: the dependent variable was the amplitude of the evoked potential $(\mathrm{mV})$ in response to the stimulus onset. The independent variables were noise exposure (noise-exposed, unexposed; between-subjects), tone position (first, second; within-subjects), timing (regular, irregular; withinsubjects), and HFS phase (pre-HFS, post-HFS; within-subjects). Tinnitus induction, GPIAS, surgical and deep brain stimulation procedures have been described in detail elsewhere. ${ }^{22}$

\section{Tinnitus induction}

Animals were anesthetized using Ketamine and Xylazine. Rats in the noiseexposed group (i) were unilaterally exposed to a $16 \mathrm{kHz}$ octave-band noise at $115 \mathrm{~dB}$ for $90 \mathrm{~min}$. Unexposed control rats (ii) were only anesthetized.

\section{Gap-Prepulse Inhibition of the Acoustic Startle}

Behavioral evidence of tinnitus was assessed before and three weeks after noise or sham exposure by GPIAS, previously described in detail. ${ }^{22}$ Freely moving rats were placed in a cylinder, on a piezo sensor. Gap and no-gap trials were alternately executed with 20 repetitions per background frequency $(10 \mathrm{kHz}$, $16 \mathrm{kHz}$ and $20 \mathrm{kHz}$ at $75 \mathrm{~dB}$ ). The startle stimulus consisted of a click sound of 
$105 \mathrm{~dB}$ intensity with $20 \mathrm{~ms}$ duration. In gap trials, a gap of $50 \mathrm{~ms}$ was added to the background sound, $100 \mathrm{~ms}$ prior to the startle stimulus. Prior to each session, the animals were acclimatized for 5 minutes and habituated to the startle sound by presenting 10 no-gap trials. To habituate the animals to the testing procedure, one complete session was performed at the beginning of the experiment. Two complete sessions per condition were conducted, each on separate testing days. The gap/no-gap ratio was calculated by dividing the amplitude per gap-startle with the mean of all startle-only trials. ${ }^{19}$

\section{Surgical procedure}

Rats were anesthetized by intraperitoneal administration of urethane ( $7.5 \mathrm{ml} / \mathrm{kg}$ loading dose and $0.3 \mathrm{ml}$ repetitive dose for maintenance) from a $20 \%$ of weight urethane solution (Sigma-Aldrich / Merck KGaA, Darmstadt, Germany). The level of anaesthesia was monitored by checking whisker and pedal reflexes. Body temperature was controlled and maintained at $37^{\circ} \mathrm{C}$ by means of a heating pad (ATC1000, World Precision Instruments, Sarasota, Florida). Rats were mounted on a stereotaxic apparatus (Stoelting Co, Illinois, USA) using hollow ear bars to allow presentation of auditory stimuli. A craniotomy was performed in order to access the left MGB which is contralateral to the noise (or sham) exposed ear. To record local field activity, a bipolar electrode was inserted into the contralateral MGB (craniocaudal $5.5 \mathrm{~mm}$, mediolateral $+3.6 \mathrm{~mm}$, dorsoventral $-6 \mathrm{~mm}$ ) according to Paxinos rat brain atlas. ${ }^{23}$ The electrode was a custom-made platinum-iridium bipolar electrode with a shaft diameter of $250 \mu \mathrm{m}$ and tip diameter of $50 \mu \mathrm{m}$ (Technomed, Beek, the Netherlands).

\section{Acquisition of evoked potentials and the auditory stimulation paradigm}

Electrophysiological recordings were performed four weeks after noise exposure. The electrode was connected to a data acquisition system (PowerLab 8/35, New South Wales, Australia) and sampled at $20000 \mathrm{~Hz}$ to record the local field potentials, using LabChart Pro 7 software (ADInstruments, Castle Hill, Australia). A band pass filter of $0.1 \mathrm{~Hz}-1 \mathrm{kHz}$ was used. External auditory stimuli were generated by a PC audio interface (0204 USB Audio Interface, E-MU systems, Dublin, Ireland), using custom-made MATLAB scripts. Auditory stimuli were amplified and presented with an Ultrasonic Dynamic Speaker (Vifa, Avisoft Bioacoustics, Berlin, Germany), calibrated with a 
modular precision sound level meter (Bruel and Kjaer 2231, San Diego, USA) and a free-field microphone (Bruel and Kjaer type 4191, San Diego, USA). The contralateral ear was sealed to block external auditory perception.

The auditory stimuli consisted of two pure tones with different pitches, the $600 \mathrm{~Hz}$ tone serving as the standard and the $660 \mathrm{~Hz}$ tone as deviant (see Figure 6.1). The stimulus sequences consisted of six blocks of binary grouped tones, with each block lasting for 1 minute. Each block thus consisted of 60 stimulus pairs. Six blocks were performed without interruption for a total of six minutes, once with regular (isochronous) timing intervals and once with irregular (jittered) intervals. Occurrence of the deviant tones was balanced across the two positions of each pair. Each stimulus lasted for $70 \mathrm{~ms}$ including $10 \mathrm{~ms}$ rise and fall times. The standard-to-deviant ratio was 4:1. These stimuli were organized into two separate arrangements, one generating a regular (predictable) and one an irregular (less predictable) timing. The latter was realized via random variation of both the interval within (intra-chunk) and the interval between consecutive pairs (inter-chunk interval). The pairs consequently either consisted of two standard tones (S1S2), or standard and then deviant tone (S1D2), or deviant then standard tone (D1S2). Each rat received an initial auditory stimulation with the regular and irregular sequence, both before and directly after HFS. A counterbalanced design was used. This setup allowed assessment of SG effects associated with pitch (stimulus type: standard or deviant), binary grouping (position in a pair: first or second), and temporal regularity (timing: regular or irregular).

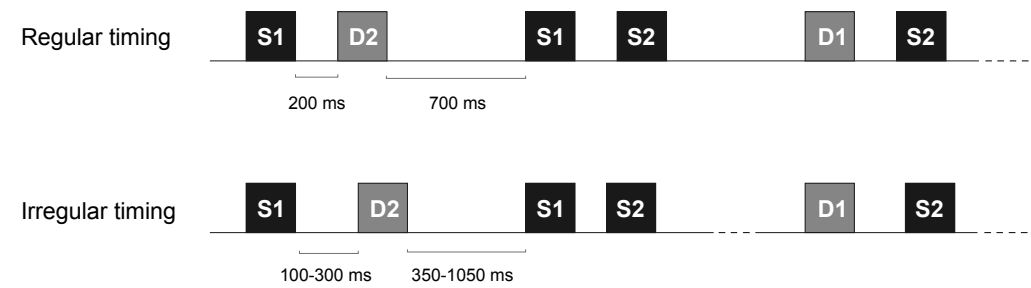

Figure 6.1 External auditory stimuli: An exemplary sequence of standard and deviant tones, depicting the length of intervals in regular and irregular timing conditions. Note that a range is designated in the irregular sequence. $\mathrm{S}=$ standard tone $(600 \mathrm{~Hz}), \mathrm{D}=$ deviant tone $(660 \mathrm{~Hz})$. 


\section{Deep brain stimulation}

MGB HFS was applied for a period of five minutes with the same bipolar electrode as used for the EP recordings. HFS $(100 \mathrm{~Hz}, 60 \mu \mathrm{s}, 100 \mu \mathrm{A}$, bipolar, monophasic square-wave pulses) was applied with a stimulator (DS8000, World Precision Instruments, Sarasota, Florida) connected to a constant-current isolator (DLS100, WPI, Sarasota, Florida). These stimulation parameters were based on previous experiments. ${ }^{20}$ Regular and irregular external auditory sound sequences were repeated, each being preceded by HFS, again using a counterbalanced design.

\section{Electrode localization}

To check for correct electrode tip placement, the rats were euthanized by decapitation while still being under general anesthesia, the brains were quickly removed and frozen in $-40^{\circ} \mathrm{C}$ 2-methyl-butane (isopentane). The tissue was serially cut by cryostat (Leica CM3050S, Wetzlar, Germany). Hematoxylineosin staining was performed to confirm appropriate electrode placement.

\section{Data processing}

Analyses were performed using the 'Letswave' toolbox ${ }^{24}$ running in MATLAB®. The signals were bandpass filtered to include frequencies between 5-30 Hz. After baseline correction, outliers were removed for a maximum of 5 epochs (from a total of 72) in the deviant group and 20 in the standard group (from a total of 288). Next, data were averaged across each event code (S1, S2, D1, D2). Amplitude of the first peak following the trigger onset was defined for further statistical comparison (Figure 6.2).

\section{Statistical analysis}

Amplitudes were not normally distributed and, consequently, were logtransformed prior to analysis. Outliers were excluded based on visual inspection, specifically values less than $-1 \mathrm{mV}$ and greater than $1 \mathrm{mV}$. The logtransformed amplitudes were fitted to a linear mixed-effects model (LMEM) with fixed factors Stimulus Type (2; Standard, Deviant), Position (2; First, Second), Noise (2; Unexposed, Noise-exposed), Timing (2; Regular, Irregular), and HFS phase (2; Pre-HFS, Post-HFS), and random effects RatID, Block, and Trial, where Trial was nested in Block, and Block was nested in RatID. Models were selected based on the lowest Akaike's Information Criteria (AIC) value 
when including the interaction term or only main effects between the fixed factors. Data were analyzed using the lmer function in the lme4 package ${ }^{25}$ for $\mathrm{R}$ software. ${ }^{26}$ Effect sizes were measured as generalized eta squared $\left(\eta G^{2}\right)$ using the aov_car function in the afex package. ${ }^{27}$ Pairwise comparisons were calculated as Tukey's Honestly Significant Difference (HSD) using the multcomp package. ${ }^{28}$ To determine evidence for the null hypothesis, Bayes Factor $t$-tests were performed using the ttestBF function of the BayesFactor package..$^{29}$ Following Jeffreys, ${ }^{30}$ Bayes Factor values were interpreted as follows: values around 1 indicates no evidence, 1-3 indicate anecdotal evidence, 3-10 indicate moderate evidence, 10-30 indicate strong evidence, 30-100 indicate very strong evidence, and greater than 100 indicates extreme evidence.

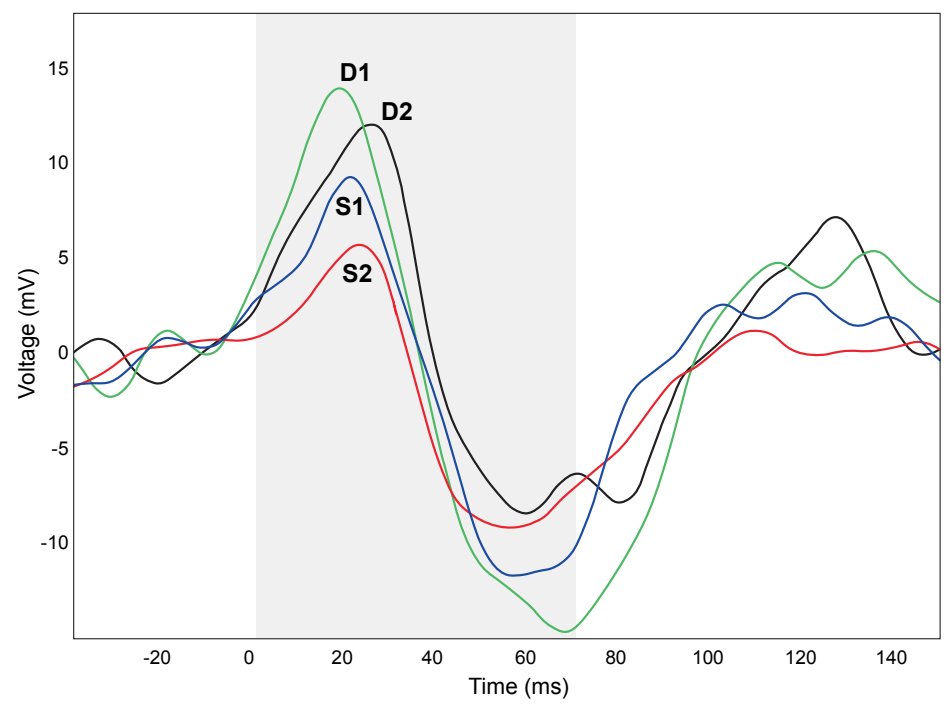

Figure 6.2 A representative mean evoked potential (EP) from a control rat at pre-HFS condition during the regular timing condition. Note that event codes (D1, D2, S1, S2) have different peak amplitudes. The grey area represents the auditory stimulus of $70 \mathrm{~ms}$ duration. 


\section{Results}

\section{Gap-Prepulse Inhibition of Acoustic Startles}

Tinnitus was assessed through GPIAS where the gap/no-gap ratios increased following noise exposure for $20 \mathrm{kHz}$ and $16 \mathrm{kHz}\left(p_{s}<0.01\right)$ but not $10 \mathrm{kHz}$ $(p=0.21)$ background sound; unexposed rats showed no significant changes ( $p s>0.14)$. These findings suggest that tinnitus was successfully induced in the noise-exposed animals. ${ }^{31}$ These GPIAS results are similar to earlier published studies from our group. ${ }^{18,19}$

\section{Evoked potentials}

Peak EP amplitude up to $70 \mathrm{~ms}$ after the trigger onset was measured to perform comparisons. The LMEM with the best model fit contained main effects of Stimulus Type and Position, and interactions between the other variables. There were significant main effects of Stimulus Type $[F(1,37256)=$ 4.20, $p=0.04), \eta_{G^{2}}=0.001$; Figure 6.3A], Position $[F(1,37255)=10.86, p<0.001$; Figure 6.3B], $\left.\eta_{G^{2}}=0.001\right]$, and Timing $\left.[F(1,37256)=12.79, p<0.001), \eta_{G^{2}}=0.002\right]$. There was a significant two-way interaction between Noise and Timing $[F(1$, 37256)=21.83, $\left.p<0.001), \quad \eta_{G^{2}}=0.003\right]$, and a significant three-way interaction between Noise, Timing, and HFS phase [F (1, 37256)=12.67, $\left.p<0.001), \eta_{G^{2}}=.002\right]$. No other main effects or interactions reached significance ( $p s>0.21)$. As shown in Figure 6.3A, deviant tones elicited significantly higher amplitudes than standard tones supporting the hypothesis that habituation occurs for stimuli that are more predictable and occur more often. Similarly, amplitudes were lower for the second stimulus in a pair compared to the first suggesting that sensory gating occurs for the second stimulus in a pair of temporally proximal tones (Figure 6.3B).

Pairwise comparisons investigating the three-way interaction between Noise, Timing, and HFS phase indicated that: only the noise-exposed rats displayed a significant decrease in amplitude from the Pre-HFS phase to the Post-HFS phase in both timing conditions $(p s<0.001)$. In contrast, the control group did not show significant changes ( $p s>0.14$; Figure $6.3 \mathrm{C}$ ). The control group showed significantly higher amplitudes for irregular timing compared to regular timing both pre- and post-HFS $(p s<0.001)$. The noise-exposed group, however, did not yield significant differences between timing conditions pre- or postHFS ( $p s>0.20$ ). These results suggest that timing-related amplitude changes only occurred under normal (control) hearing conditions and were not 
recovered in the noise-exposed rats following HFS. Finally, amplitudes were significantly higher for the noise-exposed group compared to the control group in both timing conditions in the pre-HFS phase $(p s<0.007)$. The noise-exposed group only showed greater amplitudes than controls for the post-HFS phase in the regular timing condition $(p=0.006)$ but not the irregular timing condition $(p=0.47) ;$ a one-tailed Bayes factor $\mathrm{t}$ test indicated extreme evidence that amplitudes were not higher for noise-exposed rats compared to controls postHFS in the irregular timing condition $(B F 01=151.4 \pm 0.01 \%)$. Taken together, these results suggest that noise exposure can impair timing-related SG and that HFS can restore SG to levels similar to that of controls for the unpredictable timing of auditory stimuli.
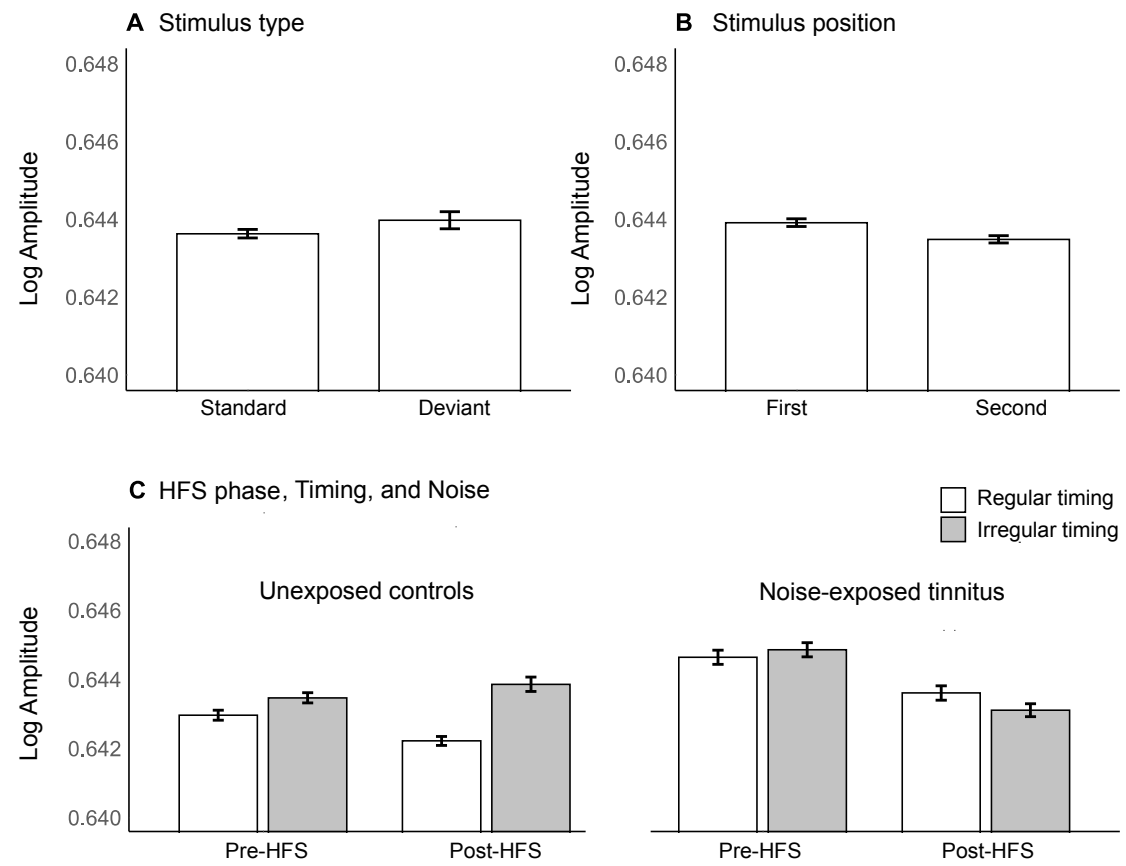

Figure 6.3 Means (+- standard error of the means) of EP amplitudes demonstrate the main effects of Stimulus type (A), and Stimulus position (B), and the three-way interaction between Timing, HFS Phase, and Noise (C). 


\section{Discussion}

The current study investigated SG in the MGB of unexposed and noiseexposed tinnitus animal model as well as the effects of HFS. Overall, our findings show the MGB's distinct role in filtering incoming auditory signals based on the predictability of two stimulus features (pitch and temporal regularity). Further, higher EP amplitudes were found in noise-exposed animals in most conditions (detailed below). Moreover, HFS of the MGB resulted in a decrease in auditory EP amplitudes selectively in noise-exposed animals.

\section{Auditory filtering capacities of MGB}

To our knowledge, this is the first study to provide evidence that auditory SG occurs at the level of the MGB in rats. Responses to deviant tones were significantly enhanced compared to standard tones (Figure 6.3A). Furthermore, responses to the second tone of a pair were significantly lower than those to the first tone (Figure 6.3B). In control animals, EP amplitudes in the irregular timing condition were significantly higher compared to the regular timing condition (Figure 6.3C). Here, the higher EP amplitude in response to a deviant tone is comparable to the mismatch responses such as the Mismatch Negativity seen in human EEG recordings. ${ }^{32}$ A previous study in anesthetized rats showed enhanced EP response to deviant tones across different sleep states (including REM and non-REM sleep) in the auditory cortex. ${ }^{33}$ Other studies reported sensitivity of EPs to deviant tones in primary auditory cortex. ${ }^{34}$ However, the role of subcortical structures in SG has not yet been elucidated. Overall, the results confirm that the MGB filters sensory information in relation to position, pitch, and temporal regularity.

\section{Sensory gating after noise exposure}

Tinnitus assessment using GPIAS confirmed that noise-exposed animals had tinnitus. Unexposed control rats exhibited higher amplitudes in the MGB for irregular timing compared to regular timing, either before or after HFS. This points to the fact that, in contrast to more popular views of cortical handling of regularity, predictive timing abilities (i.e., entrainment) already occurs at the subcortical level. In contrast, noise-exposed rats were indifferent to timing regularities, either before or after HFS. This may be due to disturbed neurophysiological activity of individual MGB neurons in noise-exposed rats. 
At a neuronal population level this might be reflected by the inability to distinguish regular and irregular timing in the noise-exposed animals, i.e. to take advantage of the full temporal predictability of the regular stimulus sequence. Thus, at the level of the MGB, noise-exposed animals are unable to distinguish temporal regularity of auditory stimuli. These results suggest that entrainment requires SG and low-level sensory processing, and cannot be restored via MGB HFS. Therefore, findings indicate that entrainment to timing regularities might be a bottom-up process that can be perturbed by disruptions to SG.

Additionally, we found a significantly higher EP amplitude in noise-exposed than control animals across all conditions except for the irregular stimulation group at post-HFS phase. This may be due to the increased synchronization of firing in subcortical auditory centers in noise-exposed animals. ${ }^{35}$ As shown before, elevated EPs might be a neural signature of tinnitus. ${ }^{36}$ The noiseexposed animals in the current experiment showed tinnitus-like behaviour, which was confirmed by GPIAS. However, prematurely linking elevated EPs in the noise-exposed animal group to tinnitus would be unwarranted. An alternative reason for increased responses may be higher levels of stress in these animals, making them more sensitive to external stimuli. Various studies confirmed alterations in burst patterns in MGB of rats with acoustic trauma which may affect the EP amplitudes of tinnitus animals relative to control animals. ${ }^{36,37}$ Furthermore, a possible confounding effect of hyperacusis cannot be ruled out. Hyperacusis often coexists with tinnitus, and both conditions might be a result of increased synchronized electrophysiological activity, which could lead to elevated auditory EP amplitudes. ${ }^{38}$ Follow-up studies are needed in order to disentangle the role of tinnitus, hyperacusis, and hearing loss on disturbed SG. The experimental design should enable to separate animals which show hyperacusis, tinnitus-like behaviour and/or hearing loss or a combination of these. Based on our findings it remains speculative whether the elevated EP amplitudes in the noise-exposed animals were related to tinnitus or hyperacusis.

From a clinical perspective it would be interesting to develop an objective biomarker for tinnitus. The use of SG as an objective biomarker has also been suggested in other disorders in which SG is abnormal. In fact, P50 abnormalities have been found in the EEG of patients suffering from schizophrenia. ${ }^{39,40}$ Although a clinical study suggests that tinnitus follows 
dysfunctional SG, further studies are needed to confirm this before SG could be considered as a diagnostic tool for tinnitus. ${ }^{10}$

\section{Effect of high frequency stimulation on sensory gating}

We found an overall significant suppressing effect of HFS on EP amplitudes in noise-exposed tinnitus animals, but not in unexposed controls. Currently, disrupted signaling as a working mechanism of HFS is the key premise of most hypotheses regarding the restoration of the neuronal physiology in various pathologies. ${ }^{41}$ HFS induces action potentials and causes neBourons to lose their ability to transmit information. ${ }^{42}$ This way, pathological oscillations can be eliminated and neural firing patters can be normalized. ${ }^{43,44}$ The current findings lend preliminary support to this hypothesis; evoked EP hyperactivity, which is a neural correlate of tinnitus, was normalized after HFS, except for stimulustiming effects. This normalization might be due to a disruption of pathological oscillations in the thalamo-cortical loop, known as thalamo-cortical dysrhythmia. ${ }^{6}$ This effect was not found in unexposed control animals, which may indicate the specific influence of HFS on aberrant neuronal firing.

\section{Conclusions}

The MGB serves as a filtering station for auditory stimulus processing. This includes filtering in relation to position, pitch, and temporal regularity. Noiseexposed animals could not distinguish temporal regularity in auditory stimuli. Furthermore, they showed an overall increase in EP activity at MGB relative to unexposed controls. HFS can suppress the increased EP amplitudes towards normal levels and could potentially restore an animal's sensory gating capacity. 


\section{References}

1. Auerbach BD, Rodrigues PV, Salvi RJ. Central gain control in tinnitus and hyperacusis. Front Neurol. 2014;5:206.

2. Norena AJ. An integrative model of tinnitus based on a central gain controlling neural sensitivity. Neurosci Biobehav Rev. 2011;35(5):1089-109.

3. Sedley W. Tinnitus: Does Gain Explain? Neuroscience. 2019;407:213-28.

4. Rauschecker JP, May ES, Maudoux A, Ploner M. Frontostriatal Gating of Tinnitus and Chronic Pain. Trends Cogn Sci. 2015;19(10):567-78.

5. Rauschecker JP, Leaver AM, Muhlau M. Tuning out the noise: limbic-auditory interactions in tinnitus. Neuron. 2010;66(6):819-26.

6. De Ridder D, Vanneste S, Langguth B, Llinas R. Thalamocortical Dysrhythmia: A Theoretical Update in Tinnitus. Frontiers in Neurology. 2015;6(124).

7. Caspary DM, Llano DA. Auditory thalamic circuits and GABAA receptor function: Putative mechanisms in tinnitus pathology. Hear Res. 2017;349:197-207.

8. Zhang Z, Liu C-H, Yu Y-Q, Fujimoto K, Chan Y-S, He J. Corticofugal Projection Inhibits the Auditory Thalamus Through the Thalamic Reticular Nucleus. Journal of Neurophysiology. 2008;99(6):2938-45.

9. Campbell J, LaBrec A, Bean C, Nielsen M, So W. Auditory Gating and Extended HighFrequency Thresholds in Normal-Hearing Adults With Minimal Tinnitus. Am J Audiol. 2019;28(1S):209-24.

10. Mohebbi M, Farhadi M, Daneshi A, Mahmoudian S. Toward An Exploration of Habituating to Tinnitus: Perspectives on Sensory Gating. J Am Acad Audiol. 2019.

11. Jones LA, Hills PJ, Dick KM, Jones SP, Bright P. Cognitive mechanisms associated with auditory sensory gating. Brain Cogn. 2016;102:33-45.

12. Soshi T, Noda T, Ando K, Nakazawa K, Tsumura H, Okada T. Impulsivity is associated with early sensory inhibition in neurophysiological processing of affective sounds. Front Psychiatry. 2015;6.

13. Li S, Ma L, Wang Y, Wang X, Li Y, Qin L. Auditory steady-state responses in primary and non-primary regions of the auditory cortex in neonatal ventral hippocampal lesion rats. PLoS One. 2018;13(2):e0192103.

14. Cromwell HC, Mears RP, Wan L, Boutros NN. Sensory gating: a translational effort from basic to clinical science. Clin EEG Neurosci. 2008;39(2):69-72.

15. Rammo R, Ali R, Pabaney A, Seidman M, Schwalb J. Surgical Neuromodulation of Tinnitus: A Review of Current Therapies and Future Applications. Neuromodulation. 2019;22(4):380-7.

16. van Zwieten G, Smit JV, Jahanshahi A, Temel Y, Stokroos RJ. Tinnitus: Is there a place for brain stimulation? Surgical neurology international. 2016;7(Suppl 4):S125-S9.

17. Smit JV, Janssen MLF, Schulze H, Jahanshahi A, Van Overbeeke JJ, Temel Y, et al. Deep brain stimulation in tinnitus: Current and future perspectives. Brain Research. 2015;1608:51-65.

18. van Zwieten G, Jahanshahi A, van Erp ML, Temel Y, Stokroos RJ, Janssen MLF, et al. Alleviation of Tinnitus With High-Frequency Stimulation of the Dorsal Cochlear Nucleus: A Rodent Study. Trends Hear. 2019;23:2331216519835080.

19. Smit JV, Janssen ML, van Zwieten G, Jahanshahi A, Temel Y, Stokroos RJ. Deep brain stimulation of the inferior colliculus in the rodent suppresses tinnitus. Brain Res. 2016;1650:118-24.

20. van Zwieten G, Janssen MLF, Smit JV, Janssen AML, Roet M, Jahanshahi A, et al. Inhibition of Experimental Tinnitus With High Frequency Stimulation of the Rat Medial Geniculate Body. Neuromodulation. 2019;22(4):416-424.

21. Ashkan K, Rogers P, Bergman H, Ughratdar I. Insights into the mechanisms of deep brain stimulation. Nature Reviews Neurology. 2017;13:548. 
22. van Zwieten G, Roberts MJ, Schaper FLVW, Smit JV, Temel Y, Janssen MIF. Noise-induced neurophysiological alterations in the rat medial geniculate body and thalamocortical desynchronization by deep brain stimulation. J Neurophysiol 2021;125(2):661-71.

23. Paxinos G, Watson C. The Rat Brain in Stereotaxic Coordinates: Hard Cover Edition: Elsevier Science; 2013.

24. André Mouraux BR, Li Hu, Giandomenico Iannetti Letswave 6 - Matlab EEG signal processing toolbox. Github repository; 2018.

25. Bates D, Mächler M, Bolker B, Walker S. Fitting Linear Mixed-Effects Models Using lme4. 2015. 2015;67(1):48.

26. R Core Team. R: A language and environment for statistical computing. Vienna, Austria: R Foundation for Statistical Computing; 2013.

27. Singmann H, Bolker B, Westfall J, Aust F. Analysis of Factorial Experiments. R package version 0.22-1. 2019.

28. Hothorn T, Bretz F, Westfall P. Simultaneous inference in general parametric models. Biom J. 2008;50(3):346-63.

29. Morey RDR, J. N. . BayesFactor: Computation of Bayes Factors for Common Designs. R package version 0.9.12-4.2. 2018.

30. Jeffreys H. Theory of Probability: Clarendon Press; 1961.

31. van Zwieten G, Roberts MJ, Schaper FL, Smit JV, Temel Y, Janssen MLF. Noise-induced neurophysiological alterations in the rat medial geniculate body and thalamocortical desynchronization by deep brain stimulation. J Neurophysiol. 2021.

32. Dehaene S, Changeux JP. Experimental and theoretical approaches to conscious processing. Neuron. 2011;70(2):200-27.

33. Nir Y, Vyazovskiy VV, Cirelli C, Banks MI, Tononi G. Auditory responses and stimulusspecific adaptation in rat auditory cortex are preserved across NREM and REM sleep. Cereb Cortex. 2015;25(5):1362-78.

34. Polterovich A, Jankowski MM, Nelken I. Deviance sensitivity in the auditory cortex of freely moving rats. PLoS One. 2018;13(6):e0197678.

35. Shore SE, Wu C. Mechanisms of Noise-Induced Tinnitus: Insights from Cellular Studies. Neuron. 2019;103(1):8-20.

36. Kalappa BI, Brozoski TJ, Turner JG, Caspary DM. Single unit hyperactivity and bursting in the auditory thalamus of awake rats directly correlates with behavioural evidence of tinnitus. J Physiol-London. 2014;592(22):5065-78.

37. Barry KM, Robertson D, Mulders WHAM. Changes in auditory thalamus neural firing patterns after acoustic trauma in rats. Hearing Res. 2019;379:89-97.

38. Chen YC, Chen GD, Auerbach BD, Manohar S, Radziwon K, Salvi R. Tinnitus and hyperacusis: Contributions of paraflocculus, reticular formation and stress. Hear Res. 2017;349:208-22.

39. Karkal R, Goyal N, Tikka S, Khanande R, Kakunje A, Khess C. Sensory gating deficits and their clinical correlates in drug-free/drug-naive patients with schizophrenia. Indian Journal of Psychological Medicine. 2018;40(3):247-56.

40. Ashley K. Smith, J. Christopher Edgar, Mingxiong Huang, Brett Y. Lu, Robert J. Thoma, Faith M. Hanlon, et al. Cognitive Abilities and 50- and 100-msec Paired-Click Processes in Schizophrenia. American Journal of Psychiatry. 2010;167(10):1264-75.

41. Chiken S, Nambu A. Mechanism of Deep Brain Stimulation: Inhibition, Excitation, or Disruption? Neuroscientist. 2016;22(3):313-22.

42. McIntyre CC, Anderson RW. Deep brain stimulation mechanisms: the control of network activity via neurochemistry modulation. J Neurochem. 2016;139 Suppl 1:338-45.

43. McConnell GC, So RQ, Hilliard JD, Lopomo P, Grill WM. Effective deep brain stimulation suppresses low-frequency network oscillations in the basal ganglia by regularizing neural firing patterns. J Neurosci. 2012;32(45):15657-68.

44. Hamani C, Moro E. Neuromodulation: a more comprehensive concept beyond deep brain stimulation. Int Rev Neurobiol. 2012;107:1-3. 



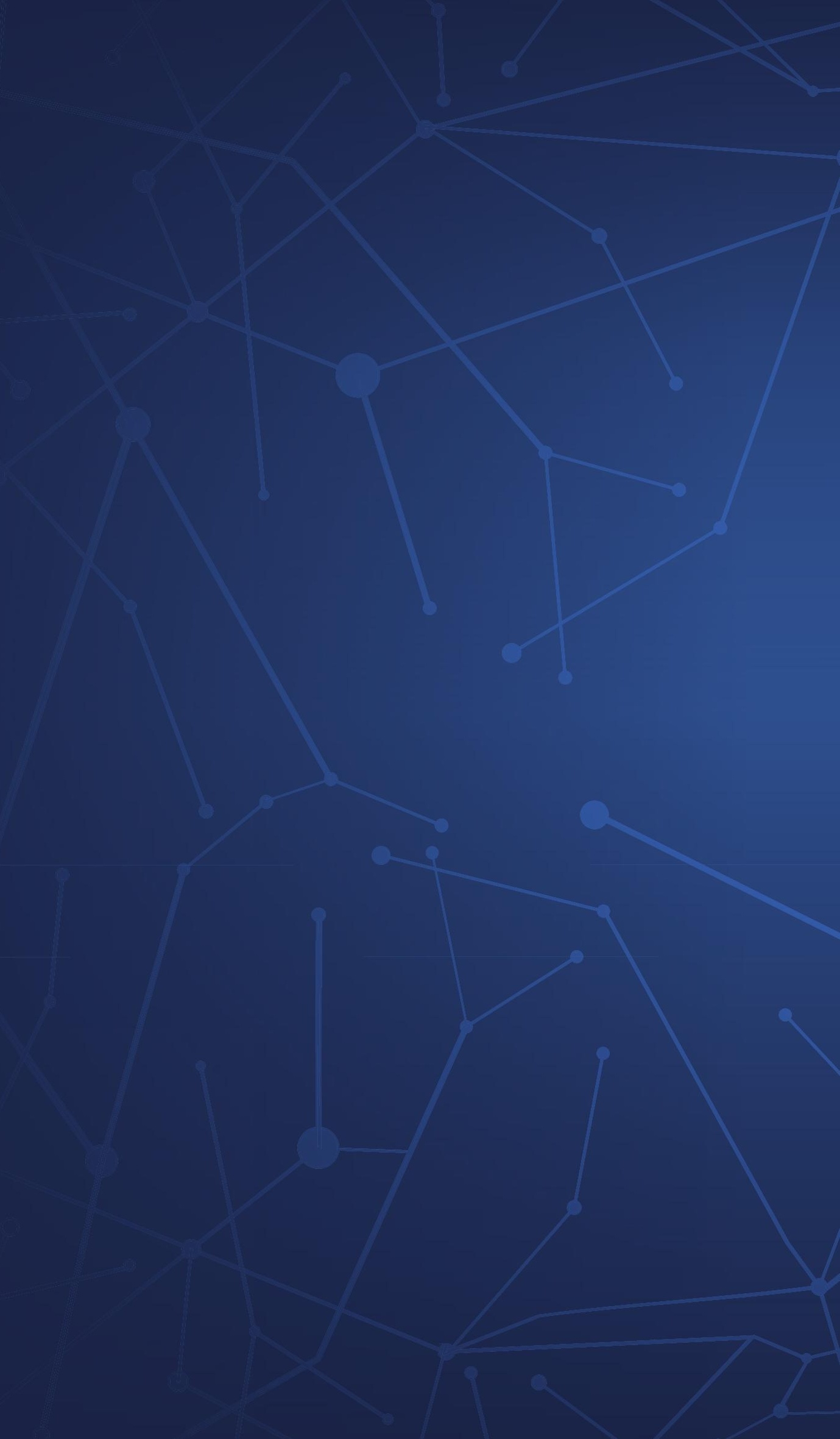




\section{PART III}

Clinical translation 



\section{Deep brain stimulation for refractory tinnitus: a protocol for a randomized double-blinded cross-over clinical pilot study}

Van Zwieten G

Devos JVP

Kotz SA

Ackermans L

Brinkmann P

Dauven L

George ELJ

Janssen AML

Kremer B

Leue $C$

Schwartze M

Temel Y

Smit JV

Janssen MLF 


\section{Abstract}

Background: Chronic tinnitus can have an immense impact on the quality of life. Despite recent treatment advances, many tinnitus patients remain refractory to them. Preclinical and clinical evidence suggest that deep brain stimulation (DBS) is as a promising treatment to suppress tinnitus. The thalamic medial geniculate body (MGB) has a key position in the tinnitus network, shows pathophysiological hallmarks of tinnitus, and is readily accessible using stereotaxy. This study will evaluate the safety and therapeutic effects of DBS in the MGB in severe tinnitus sufferers.

Methods: Bilateral DBS of the MGB will be applied in six patients with severe and refractory tinnitus. A double-blinded, randomized $2 \times 2$ crossover design (stimulation $\mathrm{ON}$ and $\mathrm{OFF}$ ) will be applied, followed by a period of six months open label follow-up. The primary focus is to assess safety and feasibility (acceptability). Secondary outcomes are tinnitus severity, measured by the Tinnitus Functional Index (TFI), tinnitus loudness and distress, hearing, cognitive and psychological functions, quality of life, and neurophysiological characteristics.

Discussion: Whilst carefully balancing risks and benefits and taking ethical considerations into account, this study explores the safety and feasibility of DBS in severe refractory tinnitus, by extensive assessment of clinical and neurophysiological outcome measures. Additionally, important insights in the underlying mechanism of tinnitus and hearing function might be found. 


\section{Introduction}

Tinnitus, commonly known as "ringing of the ears", is one of the largest health challenges in the world. ${ }^{1}$ According to a recent large survey, approximately $6.4 \%$ of Americans experience persistent tinnitus. ${ }^{2}$ Every tenth patient experiences a most extreme and debilitating form of tinnitus. Sleep deprivation, anxiety, and depression often accompany tinnitus and severely affect the patient's quality of life..$^{3-5}$ In turn, this places a huge burden on society, healthcare costs and decreases productivity. ${ }^{6}$

Subjective tinnitus has a multifactorial origin with heterogeneous patient profiles, which makes it a highly complex condition. The absence of an underlying medical cause in most cases leaves many patients without an available curative evidence-based treatment.7,8 Tinnitus combined with sensorineural hearing loss might benefit from hearing aids. However, somewhere between $22 \%$ and $80 \%$ of affected patients are adequately served by using hearing aids. 910 Current clinical practice primarily aims at reducing the impact of tinnitus by providing psychoeducation and improving coping strategies via various psychological interventions. ${ }^{11,12}$

The etiological and pathophysiological mechanisms of subjective tinnitus are complex and not fully unravelled. Most researchers agree that in nearly all tinnitus cases, there is some degree of cochlear damage, leading to deprived auditory input. ${ }^{13}$ Involvement of the somatosensory system in tinnitus has also been suggested. Excitation of somatosensory inputs of the central auditory system might even be able to modulate tinnitus severity in some cases. ${ }^{14}$ According to current theories, tinnitus is associated with increased neural activity in auditory cortices, possibly resulting from maladaptive gating 15,16 and/or increase in central gain. ${ }^{17}$ Specific neural correlates described in tinnitus models are enhanced neuronal synchrony, increased spontaneous firing, and changes in tonotopic organization. ${ }^{18}$ The medial geniculate body (MGB) of the thalamus is a major relay and gateway between midbrain and cortex, and a core structure in tinnitus pathophysiology. ${ }^{19,20}$ Preclinically, in multiple levels of the central auditory pathway from cochlear nuclei to auditory cortex and including the MGB, tinnitus related neuronal activity is similar to subthalamic nucleus activity in Parkinson's disease, i.e. enhanced spontaneous activity and bursty firing. ${ }^{21-24}$ The primary role of auditory thalamic neurons is to actively and dynamically shape neural representations of information and to control 
which information reaches the cerebral cortex..$^{25}$ Furthermore, integration of auditory and limbic information occurs within the MGB. ${ }^{26}$ Connected limbic structures, such as the amygdala and nucleus accumbens, are related to emotional and attentional symptoms of tinnitus. ${ }^{27}$ Hence, the MGB acts as a central hub in involved tinnitus networks, which makes it a promising structure for neuromodulatory approaches.

A commonly applied neuromodulation technique is deep brain stimulation (DBS). This therapy has been widely used in neurologic and neuropsychiatric disorders such as Parkinson's disease. DBS is generally applied using high frequency stimulation $(>100 \mathrm{~Hz})$, to disrupt pathological neuronal activity and oscillations. ${ }^{28,29}$ Hypothetically, this results in alteration of tinnitus perception and related distress. Further, patients, treated with DBS for a movement disorder sometimes also suffered from tinnitus. DBS of non-auditory structures in these patients led to diminished or completely suppressed tinnitus. ${ }^{30-32}$ Moreover, preclinical studies support the beneficial effects of DBS on tinnitus when applied in auditory brain areas. A reduction in tinnitus-like behavior was shown when DBS was applied in several structures along the classical auditory pathway, including the dorsal cochlear nucleus, ${ }^{33,34}$ inferior colliculus, ${ }^{35}$ and MGB. ${ }^{36}$ Importantly, no undesired side-effects occurred. DBS of the MGB did not lead to anxiety or disturbed locomotor activity. DBS of the inferior colliculi did not cause any detectable hearing impairment. ${ }^{37}$ Therefore, DBS seems to be a promising treatment for severe, refractory tinnitus. ${ }^{38-40}$ The MGB is a preferred target area next to other auditory subcortical structures, ${ }^{39}$ as the auditory thalamus is readily accessible in stereotactic surgery. Consequently, targeting the auditory thalamus bears smaller surgical risks and complications such as bleeding and potential neurological deficit. Currently, MGB DBS has not been applied in humans. The majority of patients with tinnitus can be treated with non-invasive methods, and only a small number of patients can be considered as a candidate for DBS.

The primary objective of the proposed study is to assess the safety and feasibility (acceptability) of bilateral MGB DBS in severe tinnitus. Patients with severe tinnitus, who are refractory to the standard treatment program will be included. Secondary outcomes will provide preliminary data on the effect of MGB DBS on tinnitus severity (Tinnitus Functional Index), tinnitus loudness and distress (Visual Analogue Scales), hearing (audiometry), cognition (neuropsychological test battery), quality of life and psychological functioning 
(questionnaires). Furthermore, electrophysiological data will assess fundamental aspects of auditory function and tinnitus pathophysiology.

\section{Methods}

\section{Study design}

This pilot study uses a double-blind $2 \times 2$ cross-over design in which DBS of the MGB will be compared to no stimulation (Figure 7.1).

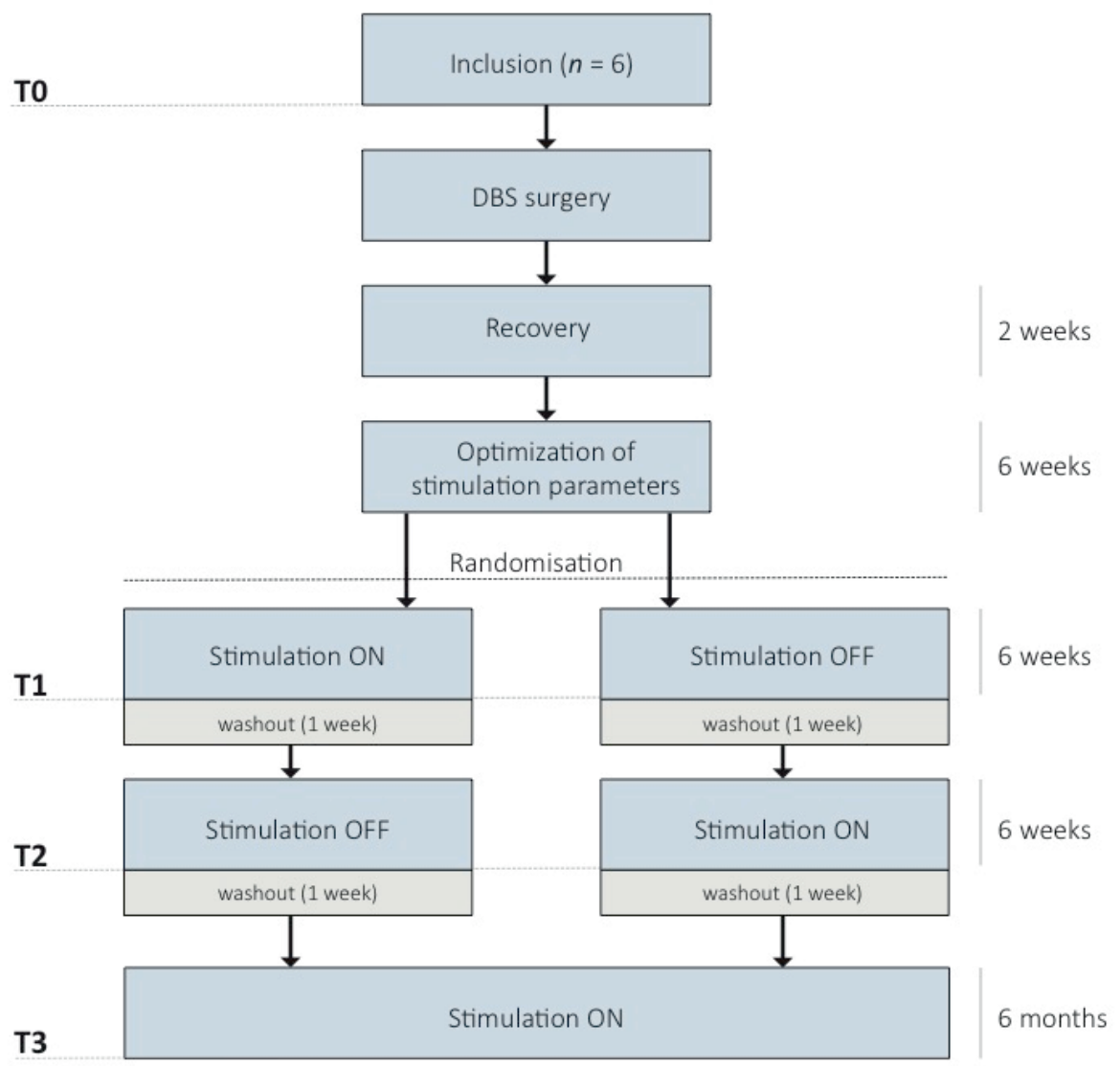

Figure 7.1 Overview of study design. 


\section{Setting}

This study will be carried out at Maastricht University Medical Center (MUMC+) in Maastricht, the Netherlands. MUMC+ is an expertise center for tinnitus, providing integrated multidisciplinary diagnostics and rehabilitation for a wide range of tinnitus patients. The Ear, Nose, and Throat department has long-standing clinical expertise and experience with developing neuromodulative therapies for tinnitus such as intracochlear devices. ${ }^{41}$ The neurosurgery department has substantial clinical and preclinical expertise in DBS. In addition to a preclinical DBS research line, ${ }^{42}$ it is well equipped to conduct clinical trials for new indications of DBS therapy such as Gilles de la Tourette syndrome. ${ }^{43}$ Acquisition of neurophysiological data, both intra- and postoperatively is standard practice and is used to unravel neural mechanisms. ${ }^{44}$

The study will be conducted according to the principles of the Declaration of Helsinki (Version 10, 2013) and in accordance with the Medical Research Involving Human Subjects Act (WMO). Ethics approval was obtained by the institutional review board. Results will be published in an international peer reviewed scientific journal and presented at scientific meetings.

\section{Recruitment and consent}

Patients are eligible to enroll if they meet the in- and exclusion criteria as outlined in Table 7.1. All patients will be evaluated and selected by a multidisciplinary team of specialists (otolaryngologists, audiologists, neurosurgeons, neurologists, psychiatrists and psychologists). Diagnostics and treatment are in accordance with national tinnitus guidelines. ${ }^{45}$ Based on the Dutch tinnitus guidelines, the tinnitus questionnaire (TQ) is used to determine tinnitus severity. ${ }^{46}$ Patients suffer severely (TQ score $\geq 47$ ), and are refractory to available treatments including cognitive behavioral therapy and hearing aids in case of hearing loss.

Patients will be recruited at the outpatient clinic of the Ear Nose Throat department. If patients give permission, they receive an information brochure. Two weeks after, researchers will contact the patients to plan a face-to-face meeting. During this meeting, a full understanding of the study protocol is ensured and additional questions are answered. When a patient needs more time to decide, the investigator plans a follow-up appointment after a few weeks. If a patient agrees to participate in the study, informed consent will be signed by the patient and investigator. If the patient meets the criteria, a second 
outpatient visit will be planned. During this visit, an intake interview will be conducted by one of the researchers, followed by a consultation with both a psychiatrist and a neurosurgeon. Then, the multidisciplinary team will form a collective decision on inclusion or exclusion. Following a positive decision of the multidisciplinary team, standard clinical workup for DBS surgery will follow. This includes conducting a brain scan and general blood examination. If inclusion criteria are still met, final inclusion will follow. Patients can leave the study at any time for any reason without any consequences. The investigators can decide to withdraw a subject from the study for urgent medical reasons.

Table 7.1 Eligibility criteria. TQ, Tinnitus Questionnaire; DBS, Deep Brain Stimulation; CI, Cochlear Implant; ABI, Auditory Brainstem Implant.

\begin{tabular}{ll}
\hline Inclusion criteria & Exclusion criteria \\
\hline Medically refractory tinnitus * & Anatomic cause of tinnitus (e.g. vestibular \\
Age 18-69 years & schwannoma, tumor, middle-ear pathology, \\
Experiencing tinnitus that is non-pulsatile and & temporal mandibular disorder) \\
uni- or bilateral & DSM-V psychiatric disorders, other than \\
Severe tinnitus (TQ score $\geq 47)$ & depression or anxiety disorder \\
Tinnitus, which is chronic (present $\geq$ two years) & Depression or anxiety disorder, manifestation \\
and stable (not intermittent $\geq$ one year) & before tinnitus onset \\
Average pure tone thresholds for 1, 2 and $4 \mathrm{kHz}$ & Cognitive impairment or coping problems \\
$<60 \mathrm{~dB}$ for each ear & Active otologic diseases \\
Willingness to participate in the study & $\begin{array}{l}\text { Pregnancy or breast-feeding } \\
\text { Active suicidal thoughts or recent attempts }\end{array}$ \\
& Life expectancy lower than two years \\
& $\begin{array}{l}\text { Implantable electronic devices that potentially } \\
\text { interfere with DBS (CI, ABI, cortical implant) }\end{array}$ \\
& General contra-indications for MRI or surgery \\
\hline
\end{tabular}

* Patient does not respond to available tinnitus treatments (e.g. sound enrichment and cognitive behavioral therapy) and is thoroughly evaluated by the multidisciplinary tinnitus team in MUMC+.

\section{Outcomes}

The time frame and methods of data acquisition are displayed in Figure 7.1 and Table 7.2, respectively. 


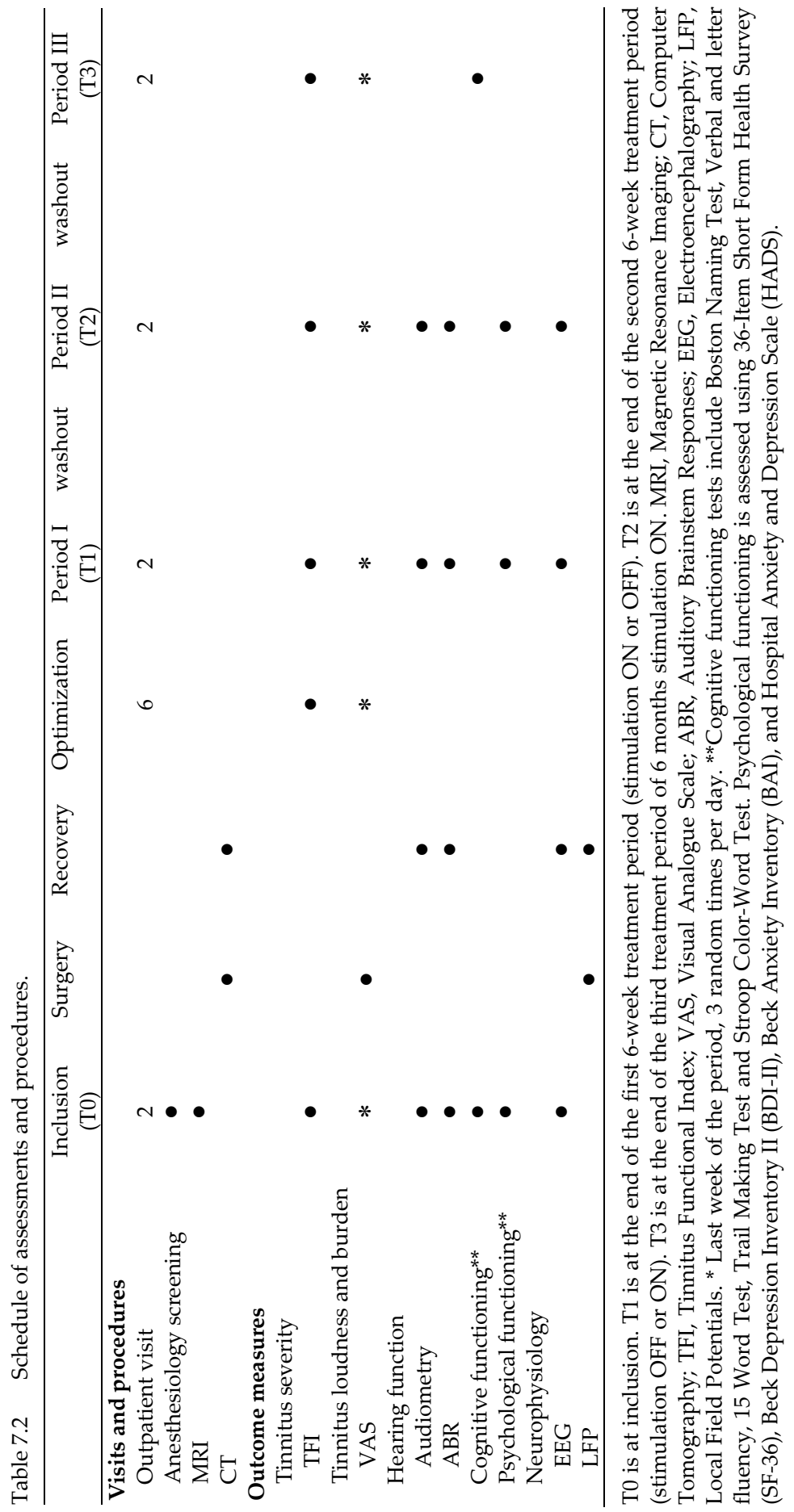




\section{Primary outcomes}

The primary focus of this pilot study is to assess safety and feasibility. Safety will be assessed by reporting the rate and grade of all surgical and stimulation induced adverse events in the study population during the study period. Feasibility will be assessed in terms of acceptability of the intervention, by taking qualitative interviews at all major timepoints (T0, T1, T2, T3) and compare satisfaction during sham stimulation and DBS.

\section{Secondary outcomes}

The secondary outcomes are meant to give preliminary data on outcomes such as tinnitus severity, hearing function, depression, anxiety, cognitive function and quality of life. Furthermore, changes in neuronal activity will be assessed by comparing electrophysiological measurements during sham stimulation and DBS.

1. Tinnitus severity will be assessed with the Tinnitus Functional Index (TFI). ${ }^{47}$ The TFI is a validated self-report questionnaire that measures overall severity of tinnitus and provides coverage of multiple tinnitus severity domains. This questionnaire is the most appropriate responsive measure of treatment-related change. The TFI is translated and validated for Dutch native speakers. ${ }^{48}$

2. Tinnitus loudness and burden will be measured by VAS. This will be done three times daily within a week, which is repeated four times during the study. Furthermore, these VAS-scores will be used to assess the effect of stimulation on tinnitus during surgery. VAS ratings for tinnitus loudness and burden are often used in both clinical practice and experimental and descriptive research as a measure of subjective symptoms. ${ }^{49}$ Both scales have been shown to correlate with the scores on Tinnitus Questionnaires..$^{50}$

3. Hearing function will be assessed with pure-tone and speech audiometry. These are clinical standard audiometric tests. Furthermore, subjective hearing will be evaluated using narratives.

4. Cognitive functioning will be measured using a validated test battery for standard DBS care. These include the Boston Naming Test, Verbal Fluency, Letter Fluency, 15 Words Test, Trail Making Test part A and B, and the Stroop Color-Word Test.

5. Quality of life and psychological functioning will be assessed by the following psychological questionnaires: 36-Item Short Form Health Survey 
(SF-36), Beck Depression Inventory II (BDI-II), Beck Anxiety Inventory (BAI), and Hospital Anxiety and Depression Scale (HADS).

6. Neurophysiological measurements: electrophysiological data and auditory brainstem responses will be recorded before and after surgery (T0 and recovery) and at the end of the treatment periods I and II (T1 and T2). Furthermore, local field potentials (LFP) will be recorded during surgery and before implantation of the pulse generator. Details are described under 'Neurophysiological assessments'.

\section{Intervention}

\section{Implantation of DBS electrodes and internal pulse generator}

A two-staged surgery will be performed. First, bilateral DBS electrodes will be implanted. Following a standard stereotactic surgical procedure, DBS electrodes will be inserted in the MGB of the thalamus and monitored by radiological and electrophysiological measures. The placement will be conducted under local anesthesia. A CT cerebrum will be carried out and fused with a pre-operative MRI in order to plan the exact trajectory. First, a microelectrode (InoMed, Emmendingen, Germany) will be inserted. Neurophysiological recordings will be performed in 0.5 to $1 \mathrm{~mm}$ steps from 10 $\mathrm{mm}$ above and maximally $5 \mathrm{~mm}$ below the target with a multi-channel system (InoMed, Emmendingen, Germany). ${ }^{51}$ Simultaneously, at each step, a sequence of auditory stimuli will be presented, which is designed based on known signal processing characteristics of the thalamus ${ }^{52}$ and to maximize the likelihood of evoking a reliable responses. The amplitude of these responses relative to spontaneous activity will be used to confirm electrode placement. After the identification of the ventral and dorsal border of the MGB, test stimulation will be applied using $130 \mathrm{~Hz}$, pulse width of $120 \mu \mathrm{s}$ and a current starting from $0.5 \mathrm{~V}$ up to $5 \mathrm{~V}$, or until undesired side-effects occur. Stimulation amplitude will be adjusted stepwise. The patient will be asked repeatedly to rate the loudness and burden of the tinnitus sound using VAS. Furthermore, the neurologist will test for undesired side-effects. The stimulation electrode will be placed once an optimal effect and acceptable or absent side-effects are reached. After placement and fixation of both stimulation electrodes (Medtronic, model 3389) the stereotactic frame will be removed and electrodes will be externalized to enable the recording of LFPs postoperatively. 
After surgery, the patient will be transferred to a neurosurgical medium care unit. On the second post-operative day a CT cerebrum will be made to confirm the definite electrode positioning. After one or two days the pulse generator (Medtronic, Activa PC model 37601) will be subcutaneously placed under general anesthesia and the electrodes will be connected. One or two days postoperative, the patient can be discharged. After the end of the trial, followup as standard DBS care will be provided.

\section{Stimulation parameters}

The following stimulation parameters will be used as minimum and maximum values during the optimization period to determine optimal stimulation parameters: stimulation frequency $(2-200 \mathrm{~Hz})$, pulse width $(60-450 \mu \mathrm{s})$, and voltage (starting from 0 to a maximum of $5 \mathrm{~V}$ ). In case of stimulation induced side-effects, bilateral stimulation can be adapted to unilateral stimulation. During treatment, patients will visit the outpatient clinic weekly. Following the treatment episode, there will be a one-week period for washout of a possible residual therapeutic effect.

\section{Neurophysiological assessments}

EEG will be recorded with surface electrodes applied to the scalp in accordance with the 10-20 international system standard. Just before implantation of the pulse generator, cables are externalized, which enables simultaneous recordings of LFPs from the depth electrodes in the MGB.

For each session, several recordings with and without auditory stimulation will be obtained. In the first phase, resting-state activity will be recorded with eyes open and eyes closed. These initial recordings serve to establish baselines for task-independent neural oscillatory activity, network activity, and coherence (assessed on the basis of dominant spontaneous low-frequency oscillations and their variability). In the second phase, auditory brainstem responses will be recorded using a standard protocol to probe auditory brainstem function before and after surgery. In the third phase, activity will be recorded during experimental auditory stimulation. The respective measures will allow assessing of basic characteristics of auditory function in general, and sensory gating in particular (i.e., adaptive filtering based on predictable feature-based and temporal information) in accordance with the adopted model. These measures have been previously obtained via surface EEG recordings in 
humans ${ }^{53,54}$ and preliminary data show comparable responses recorded from the thalamus in the animal model.

Taken together, these measures allow assessing fundamental signal coding (linear vs. event-related), time-course (temporal relation of thalamic and surface responses), and functional (deviance processing, regularity, predictability, gating) aspects of auditory function. Dysfunctional processing would correspondingly be indexed by desynchronization, lack of suppression effects, temporal decoupling (reduced correlation between thalamic and surface responses), and overall high variability.

\section{Randomization and blinding}

Randomization will be performed directly after the period of stimulation optimization by an independent institution, the Clinical Trial Center Maastricht (CTCM). Patients will be randomly assigned to one of the two study groups. The investigator who adjusts DBS parameters cannot be blinded. All other investigators and the patient are blinded to the study group. In case the patients are considered unblinded due to the nature of the stimulation the protocol will be carried out as planned. Only in the case of medical concerns, the patient and investigators will be unblinded in order to provide the care needed.

\section{Data collection and management}

Data handling will be organized according to the "FAIR guiding principles for scientific data management and stewardship". ${ }^{55}$ This will be done in cooperation with the CTCM, which is a local center that facilitates human research. Data will be collected by data entry in Castor EDC; a cloud-based, password protected data management system providing a back-up on a secured server. Audit trailing provides basic data to backtrack a file to its origin. Paper versions of questionnaires will be kept in a locked closet in the research office. All data will be anonymously coded. The key will be available to the project leader only. Data collection is monitored by the CTCM; a specific monitor is appointed to the study who will follow-up the progress and adherence to the protocol. The monitor will perform periodical checks. A data safety monitoring board, comprising of a statistician and two neurologists, is instituted which periodically reviews and evaluates the accumulated study data concerning participant safety, study conduct, progress, and efficacy. The data safety monitoring board receives and reviews the progress and acquired 
data of this trial and provides the research team with advice on the conduct of the trial.

\section{Statistical analyses}

The safety and feasibility outcomes will be reported descriptively and narratively. Descriptive statistics with $95 \%$ confidence intervals will be used to present preliminary data of secondary outcomes such as tinnitus (severity, loudness and distress), anxiety, depression, hearing function, quality of life and cognitive functioning. These data will provide some insight into population characteristics and an indication of potential changes in mean scores between the intervention and sham stimulation.

\section{Sample size}

As this is a pilot study, no formal sample size calculation was performed. Based on previous first-in-human DBS studies, ${ }^{56,57}$ we expect to adequately address the safety and the proof of concept purpose of the study by including six patients. Furthermore, this number of patients will enable collection of preliminary results that will provide meaningful information about differences between the intervention and sham stimulation. In case of the withdrawal of a patient or in case of incomplete data there will be no replacement of individual subjects.

\section{Patient and public involvement}

Patients from the Dutch tinnitus support group were involved in the development of this protocol. During information meetings, aspects of the study were discussed such as feasibility and eligibility criteria. Furthermore, patients were involved in the development of the patient information.

\section{Discussion}

One of the main ethical considerations in this study is balancing risks and benefits. The potential benefit of the intervention is a reduction of tinnitus loudness and tinnitus burden. Risks of this minimally invasive and reversible form of functional neurosurgery are surgery related complications (e.g. cerebral haemorrhage or infarction, CSF leak, seizures, meningitis or 
encephalitis), hardware failure (e.g. lead rupture, extension fibrosis, device migration) and stimulation related side-effects. These latter effects are unknown as clinical MGB DBS has not been performed before. The function of the MGB is primarily hearing thus other side-effects than hearing-related effects are less likely. Animal studies did not show hearing loss, anxiety or locomotion related side-effects in DBS in subcortical auditory structures. $33,36,37$ We also reviewed possible side-effects based on the brain structures surrounding the MGB. The MGB is located posterior to the subthalamic nucleus. The MGB is $8 \mathrm{~mm}$ wide, $6 \mathrm{~mm}$ long and $6.5 \mathrm{~mm}$ high with its largest volume at $-3.5 \mathrm{~mm}$ from the AC-PC plane. Considering the relatively large size of the MGB, current spread outside the MGB is unlikely if the DBS electrode is positioned in the center. Neighboring structures of the MGB are structures similar to other commonly used stimulation targets. Current spread to the anterior side of the MGB could result in internal capsule effects. These side effects are known from STN DBS treatment for dystonia and Parkinson's disease. The possible side effects of internal capsule stimulation are dysarthria, muscle contractions, and gaze paresis. Posterior to the MGB the ventricle can be found. Possible side effects are known from ANT DBS which has also a border at the ventricle. Antero-medially the sensory thalamus is located which is targeted when performing DBS to treat pain. More medially the fields of forel are located. Possible side effects are known from STN DBS in which current spread also may occur to the fields of forel which can result in disturbances of speech, postural stability and gait. Laterally to the MGB the optic tract is located. We know from GPi DBS that stimulation of the optic tract elicits phosphenes. All these side effects from current spread to surrounding regions are elicited by stimulation and thus reversible. Taking this together, MGB DBS could induce changes in auditory sensation, and there is a slight risk for side-effects due to current spread. In case of undesired side-effects, stimulation parameters can be adapted or stimulation can be turned off. The principal investigator will immediately inform the data safety monitoring board and the medical ethical committee in case of serious side-effect.

In relation to correctly and carefully evaluate risks and benefits, patient recruitment and extensive informed consent are crucial steps. The primary inclusion criterion is that patients need to be refractory to current treatment (e.g. cognitive behavioral therapy); second, sufficient hearing is required and patients cannot be candidates for cochlear implants. Last, motivation and written informed consent of patients to undergo DBS for tinnitus is essential. A questionnaire study showed that about $20 \%$ of tinnitus patients is willing to 
undergo DBS surgery in case of a $50 \%$ chance of successful treatment..$^{58}$ Further, patients would be willing to pay 20 times their monthly income to be treated. Most patients would accept a risk of mild side-effects, and almost half of patients would accept a risk of severe side effects. ${ }^{58}$ A caveat in patient selection is that desperate patients might see this experimental treatment as a last resort and rush through the informed consent. ${ }^{59}$ These circumstances make this group of patients vulnerable and inclusion should carefully be contemplated when informed consent is discussed. 


\section{References}

1. Henry JA, Dennis KC, Schechter MA. General review of tinnitus: prevalence, mechanisms, effects, and management. J Speech Lang Hear Res. 2005;48(5):1204-35.

2. American National Health and Nutrition Examination Survey, Audiometry section (AUQ_G). 2011-2012.

3. Andersson G, Freijd A, Baguley DM, Idrizbegovic E. Tinnitus distress, anxiety, depression, and hearing problems among cochlear implant patients with tinnitus. J Am Acad Audiol. 2009;20(5):315-9.

4. Langguth B, Landgrebe M, Kleinjung T, Sand GP, Hajak G. Tinnitus and depression. World J Biol Psychiatry. 2011;12(7):489-500.

5. Zoger S, Svedlund J, Holgers KM. Relationship between tinnitus severity and psychiatric disorders. Psychosomatics. 2006;47(4):282-8.

6. Maes IH, Cima RF, Vlaeyen JW, Anteunis LJ, Joore MA. Tinnitus: a cost study. Ear Hear. 2013;34(4):508-14.

7. Langguth $B$, Kreuzer PM, Kleinjung $T$, De Ridder D. Tinnitus: causes and clinical management. Lancet Neurol. 2013;12(9):920-30.

8. Cima RFF, Mazurek B, Haider H, Kikidis D, Lapira A, Norena A, et al. A multidisciplinary European guideline for tinnitus: diagnostics, assessment, and treatment. HNO. 2019;67(Suppl 1):10-42.

9. Kochkin S, Tyler R. Tinnitus treatment and the effectiveness of hearing aids: hearing care professional perceptions. Hearing Review. 2008;15(13):14-8.

10. Trotter MI, Donaldson I. Hearing aids and tinnitus therapy: a 25-year experience. J Laryngol Otol. 2008;122(10):1052-6.

11. Jastreboff PJ. Tinnitus retraining therapy. Br J Audiol. 1999;33(1):68-70.

12. Cima RF, Andersson G, Schmidt CJ, Henry JA. Cognitive-behavioral treatments for tinnitus: a review of the literature. J Am Acad Audiol. 2014;25(1):29-61.

13. Eggermont JJ, Roberts LE. The neuroscience of tinnitus: understanding abnormal and normal auditory perception. Front Syst Neurosci. 2012;6:53.

14. Levine RA, Nam EC, Oron Y, Melcher JR. Evidence for a tinnitus subgroup responsive to somatosensory based treatment modalities. Prog Brain Res. 2007;166:195-207.

15. Durai M, Sanders M, Kobayashi K, Searchfield GD. Auditory Streaming and Prediction in Tinnitus Sufferers. Ear Hear. 2019;40(2):345-57.

16. Rauschecker JP, May ES, Maudoux A, Ploner M. Frontostriatal Gating of Tinnitus and Chronic Pain. Trends Cogn Sci. 2015;19(10):567-78.

17. Norena AJ. An integrative model of tinnitus based on a central gain controlling neural sensitivity. Neurosci Biobehav Rev. 2011;35(5):1089-109.

18. Eggermont JJ. Pathophysiology of tinnitus. Prog Brain Res. 2007;166:19-35.

19. Leaver AM, Renier L, Chevillet MA, Morgan S, Kim HJ, Rauschecker JP. Dysregulation of limbic and auditory networks in tinnitus. Neuron. 2011;69(1):33-43.

20. Rauschecker JP, Leaver AM, Muhlau M. Tuning out the noise: limbic-auditory interactions in tinnitus. Neuron. 2010;66(6):819-26.

21. Bergman H, Wichmann T, Karmon B, DeLong MR. The primate subthalamic nucleus. II. Neuronal activity in the MPTP model of parkinsonism. J Neurophysiol. 1994;72(2):507-20.

22. Benazzouz A, Breit S, Koudsie A, Pollak P, Krack P, Benabid AL. Intraoperative microrecordings of the subthalamic nucleus in Parkinson's disease. Mov Disord. 2002;17 Suppl 3:S145-9.

23. Janssen ML, Zwartjes DG, Tan SK, Vlamings R, Jahanshahi A, Heida $T$, et al. Mild dopaminergic lesions are accompanied by robust changes in subthalamic nucleus activity. Neurosci Lett. 2012;508(2):101-5. 
24. Kalappa BI, Brozoski TJ, Turner JG, Caspary DM. Single unit hyperactivity and bursting in the auditory thalamus of awake rats directly correlates with behavioural evidence of tinnitus. J Physiol. 2014;592(22):5065-78.

25. Bartlett EL. The organization and physiology of the auditory thalamus and its role in processing acoustic features important for speech perception. Brain Lang. 2013;126(1):29-48.

26. Kraus KS, Canlon B. Neuronal connectivity and interactions between the auditory and limbic systems. Effects of noise and tinnitus. Hear Res. 2012;288(1-2):34-46.

27. De Ridder D, Vanneste S, Weisz N, Londero A, Schlee W, Elgoyhen AB, et al. An integrative model of auditory phantom perception: tinnitus as a unified percept of interacting separable subnetworks. Neurosci Biobehav Rev. 2014;44:16-32.

28. Grill WM, Snyder AN, Miocinovic S. Deep brain stimulation creates an informational lesion of the stimulated nucleus. Neuroreport. 2004;15(7):1137-40.

29. Chiken S, Nambu A. Disrupting neuronal transmission: mechanism of DBS? Front Syst Neurosci. 2014;8:33.

30. Cheung SW, Larson PS. Tinnitus modulation by deep brain stimulation in locus of caudate neurons (area LC). Neuroscience. 2010;169(4):1768-78.

31. Shi Y, Burchiel KJ, Anderson VC, Martin WH. Deep brain stimulation effects in patients with tinnitus. Otolaryngol Head Neck Surg. 2009;141(2):285-7.

32. Smit JV, Janssen ML, Engelhard M, de Bie RM, Schuurman PR, Contarino MF, et al. The impact of deep brain stimulation on tinnitus. Surg Neurol Int. 2016;7(Suppl 35):S848-S54.

33. van Zwieten G, Jahanshahi A, van Erp ML, Temel Y, Stokroos RJ, Janssen MLF, et al. Alleviation of Tinnitus With High-Frequency Stimulation of the Dorsal Cochlear Nucleus: A Rodent Study. Trends Hear. 2019;23:2331216519835080.

34. Luo H, Zhang X, Nation J, Pace E, Lepczyk L, Zhang J. Tinnitus suppression by electrical stimulation of the rat dorsal cochlear nucleus. Neurosci Lett. 2012;522(1):16-20.

35. Smit JV, Janssen ML, van Zwieten G, Jahanshahi A, Temel Y, Stokroos RJ. Deep brain stimulation of the inferior colliculus in the rodent suppresses tinnitus. Brain Res. 2016.

36. van Zwieten G, Janssen MLF, Smit JV, Janssen AML, Roet M, Jahanshahi A, et al. Inhibition of Experimental Tinnitus With High Frequency Stimulation of the Rat Medial Geniculate Body. Neuromodulation. 2018.

37. Smit JV, Jahanshahi A, Janssen MLF, Stokroos RJ, Temel Y. Hearing assessment during deep brain stimulation of the central nucleus of the inferior colliculus and dentate cerebellar nucleus in rat. PeerJ. 2017;5:e3892.

38. Smit JV, Janssen ML, Schulze H, Jahanshahi A, Van Overbeeke JJ, Temel Y, et al. Deep brain stimulation in tinnitus: current and future perspectives. Brain Res. 2015;1608:51-65.

39. van Zwieten G, Smit JV, Jahanshahi A, Temel Y, Stokroos RJ. Tinnitus: Is there a place for brain stimulation? Surg Neurol Int. 2016;7(Suppl 4):S125-9.

40. Rammo R, Ali R, Pabaney A, Seidman M, Schwalb J. Surgical Neuromodulation of Tinnitus: A Review of Current Therapies and Future Applications. Neuromodulation. 2018.

41. Arts RA, George EL, Griessner A, Zierhofer C, Stokroos RJ. Tinnitus Suppression by Intracochlear Electrical Stimulation in Single-Sided Deafness: A Prospective Clinical Trial Part I. Audiol Neurootol. 2015;20(5):294-313.

42. Tan S, Vlamings R, Lim L, Sesia T, Janssen ML, Steinbusch HW, et al. Experimental deep brain stimulation in animal models. Neurosurgery. 2010;67(4):1073-9; discussion80.

43. Ackermans L, Duits A, van der Linden C, Tijssen M, Schruers K, Temel Y, et al. Double-blind clinical trial of thalamic stimulation in patients with Tourette syndrome. Brain. 2011;134(Pt 3):832-44.

44. Janssen ML, Zwartjes DG, Temel Y, van Kranen-Mastenbroek V, Duits A, Bour LJ, et al. Subthalamic neuronal responses to cortical stimulation. Mov Disord. 2012;27(3):435-8.

45. Joustra J, Buwalda J, Cima R, Free RH, Hofman R, De Kleine E, et al. Richtlijn Tinnitus. Nederlandse Vereniging voor Keel -Neus -Oorheelkunde en Heelkunde van het Hoofd Halsgebied. 2016. 
46. Hallam RS, Jakes SC, Hinchcliffe R. Cognitive variables in tinnitus annoyance. Br J Clin Psychol. 1988;27 ( Pt 3):213-22.

47. Meikle MB, Henry JA, Griest SE, Stewart BJ, Abrams HB, McArdle R, et al. The tinnitus functional index: development of a new clinical measure for chronic, intrusive tinnitus. Ear Hear. 2012;33(2):153-76.

48. Rabau S, Wouters K, Van de Heyning P. Validation and translation of the Dutch tinnitus functional index. B-ENT. 2014;10(4):251-8.

49. Cline ME, Herman J, Shaw ER, Morton RD. Standardization of the visual analogue scale. Nurs Res. 1992;41(6):378-80.

50. Adamchic I, Langguth B, Hauptmann C, Tass PA. Psychometric evaluation of visual analog scale for the assessment of chronic tinnitus. Am J Audiol. 2012;21(2):215-25.

51. Schaper F, Zhao Y, Janssen MLF, Wagner GL, Colon AJ, Hilkman DMW, et al. Single-Cell Recordings to Target the Anterior Nucleus of the Thalamus in Deep Brain Stimulation for Patients with Refractory Epilepsy. Int J Neural Syst. 2018:1850012.

52. Sherman SM. Thalamic relay functions. Prog Brain Res. 2001;134:51-69.

53. Schwartze M, Farrugia N, Kotz SA. Dissociation of formal and temporal predictability in early auditory evoked potentials. Neuropsychologia. 2013;51(2):320-5.

54. Schwartze M, Tavano A, Schroger E, Kotz SA. Temporal aspects of prediction in audition: cortical and subcortical neural mechanisms. Int J Psychophysiol. 2012;83(2):200-7.

55. Wilkinson MD, Dumontier M, Aalbersberg IJ, Appleton G, Axton M, Baak A, et al. The FAIR Guiding Principles for scientific data management and stewardship. Sci Data. 2016;3:160018.

56. Koek RJ, Langevin JP, Krahl SE, Kosoyan HJ, Schwartz HN, Chen JW, et al. Deep brain stimulation of the basolateral amygdala for treatment-refractory combat post-traumatic stress disorder (PTSD): study protocol for a pilot randomized controlled trial with blinded, staggered onset of stimulation. Trials. 2014;15:356.

57. Cheung SW, Racine CA, Henderson-Sabes J, Demopoulos C, Molinaro AM, Heath S, et al. Phase I trial of caudate deep brain stimulation for treatment-resistant tinnitus. J Neurosurg. 2019:1-10.

58. Smit JV, Pielkenrood BJ, Arts R, Janssen ML, Temel Y, Stokroos RJ. Patient Acceptance of Invasive Treatments for Tinnitus. Am J Audiol. 2018;27(2):184-96.

59. Grant RA, Halpern CH, Baltuch GH, O'Reardon JP, Caplan A. Ethical considerations in deep brain stimulation for psychiatric illness. J Clin Neurosci. 2014;21(1):1-5. 
General discussion 



\section{General discussion}

With the studies presented in this thesis, we aimed to expand the knowledge on central mechanisms in tinnitus neuropathophysiology, and we investigated the efficacy and working mechanisms of deep brain stimulation (DBS) of the auditory pathway in an animal model of tinnitus. First, we will discuss the preclinical setup and the limitations of our approach. Next, we will elaborate on a preferred target for DBS in tinnitus. Furthermore, the rationale for DBS from an electrophysiological perspective will be discussed. Finally, we will contemplate on cautious translation of these findings into clinical practice and give future recommendations.

\section{Methodological considerations of the noise-exposed animal model}

In our studies, we used acoustic overexposure to induce tinnitus in rats. Compared to the salicylate induced tinnitus model, in which administration of high-dose salicylates is used, the noise-exposed animal model has some advantages. First, it mimics the human etiology with its physiological mechanisms better, and is therefore more clinically relevant. Furthermore, unilateral noise-exposure usually preserves hearing thresholds in one ear, whereas salicylates induce bilateral hearing loss, which hampers tinnitus assessment. Last, noise-exposure induces a more chronic tinnitus state compared to salicylates. These reasons make acoustic over-exposure the preferred method, especially in longitudinal studies, such as our experiments on the effect of DBS. Unfortunately, no rodent tinnitus model fully represents the human situation. One of the more apparent contrasts is that in preclinical research a homogenous tinnitus is induced. As our gap-prepulse inhibition of the acoustic startle reflex (GPIAS) results suggest, this is a 16 to $20-\mathrm{kHz}$ puretone continuous noise. In humans, tinnitus is a highly heterogenous phenomenon. Therefore, our preclinical results should be extrapolated with care and can probably only be extrapolated to subsets of patients within the tinnitus population.

Tinnitus was assessed by the GPIAS paradigm. We chose this reflexive method as its lasting effect is best suited for repetitive testing in a longitudinal study, as we did in our experiments. Next to reflexive models, tinnitus can also be assessed with operant models. Operant models rely on auditory perception 
and are believed to represent tinnitus best. Rats need to be trained, after which lick or lever pressing suppression or two-choice operant conditioning indicate whether rats perceive certain sounds. Reflexive models, i.e. GPIAS paradigm, rely on unconditioned acoustic startle reflexes, which primarily depend on brainstem circuits. All tinnitus models, including the noise-exposed animal model, are known to not only induce tinnitus, but additionally hearing loss and sometimes hyperacusis. These confounders are a main concern in all animal tinnitus research and the reason why results of GPIAS tests should be interpreted cautiously in this respect. To minimize the confounding effect of hearing loss, we used unilateral noise exposure. We confirmed that the contralateral ear did not show elevated hearing thresholds using auditory brainstem responses (ABR). Though, $A B R$ results should be interpreted conservatively, as they do not reflect actual sound perception. In a previous study from our group, ABR findings were confirmed using a sound induced prepulse inhibition paradigm. ${ }^{1}$ After tinnitus induction using a unilateral 16$\mathrm{kHz}$ octave-banded noise, we persistently found unilateral hearing loss in all frequencies, and the GPIAS paradigm specifically revealed increased gap:nogap ratios in the $16-\mathrm{kHz}$ and $20-\mathrm{kHz}$ band. This indicates that the effect of unilateral hearing loss on gap:no-gap ratios is nonessential.

Regarding the confounding role of hyperacusis, analysis of absolute startle forces could give further information. In order to prevent loss of implanted electrodes during attachment to the stimulation cable, our setup allowed animals to freely move in the startle chamber. Consequently, absolute startle amplitudes could not validly be analyzed, as absolute startle amplitudes are highly dependent on the location of the rat on the platform. Therefore, the exact role of hyperacusis as a confounder on GPIAS results was not determined.

A debatable limitation of our study is that we used group means instead of comparing individual tinnitus positive to negative animals. Recently, it has become more common to define tinnitus positive and tinnitus negative animals after noise exposure. This allows to compare animals with hearing loss and tinnitus, to subjects with hearing loss only. It has been estimated that $30-70 \%$ of the subjects develop tinnitus. However, we argue that tinnitus should be seen as a continuous variable, as it exists in multiple degrees of severity and loudness. Defining a cut-off value could induce a type 2 error, which could lead to a rejection of a true null hypothesis. 
Altogether, we prefer the noise-exposed animal model to gain insight on neurophysiological mechanisms of tinnitus and to assess the effect of DBS on tinnitus. In our behavioral studies, GPIAS testing was the favorable method to assess tinnitus severity. Still, it remains debatable to what degree GPIAS results reflect actual perception of tinnitus. Therefore, GPIAS results should be interpreted carefully and ideally, the confounders hearing loss and hyperacusis should be objectified and taken into account. For future preclinical studies, we recommend using Z-scores to measure tinnitus severity in rodents. To this end, an optimized set-up with restrained rats in order to minimize variability in the acquisition of absolute startle response amplitudes is a necessity.

\section{Exploring DBS in the auditory circuit}

Electrical stimulation of multiple targets within and outside the auditory pathway has shown to suppress tinnitus in experimental settings. In patients who underwent DBS to treat movement disorders, a concurrent reduction of their co-existing tinnitus was shown. ${ }^{2,3} \mathrm{~A}$ handful of case reports and human case studies has shown that electrical stimulation of varying non-auditory structures can attenuate tinnitus: a locus of caudate neurons (referred to as area LC), ${ }^{4,5}$ ventral anterior limb of the internal capsule. ${ }^{6}$ In a more recent phase 1 clinical trial caudate nucleus stimulation in 6 refractory tinnitus patients was carried out. The clinical benefits seemed promising, a tinnitus reduction could be observed and there seemed to be no change in hearing level.5,7 Yet, the number of studies is small and the chosen targets are often based on coincidental findings. Our strategy is to aim on suppression of the actual tinnitus sound, instead of comorbidities such as depression or anxiety, by targeting the "core" tinnitus network, which is the central auditory pathway.

Our results, as presented in chapter 3 and 4 , show first evidence that bilateral high frequency stimulation (HFS) of both the dorsal cochlear nucleus (DCN) and medial geniculate body (MGB) suppresses tinnitus-like behavior in the noise-induced tinnitus animal model. Previously, it has been shown that HFS of the inferior colliculus (IC) suppresses tinnitus-like behavior in an animal model. ${ }^{8}$ These findings support our hypothesis that neuromodulation within multiple structures of the tinnitus network can alleviate tinnitus. Similarly, symptoms of Parkinson's disease are also reduced by DBS of different targets within the cortico-basal ganglia network; as thalamic, subthalamic and globus pallidus DBS have proven to be effective. In line with this, we argued that DBS 
disrupts aberrant network activity, and can sort effect at any position within the pathological circuit. The effect size, the electrical current needed to obtain a positive effect, as well as the occurrence of stimulation induced side-effects possibly differs per target. These factors, together with the best stereotactic approach, determine the therapeutic window and define the target of preference. Due to the lack of comparative studies, firm conclusions on differences in efficacy of the different targets along the auditory pathway to suppress tinnitus cannot be drawn.

Preliminary findings on auditory brainstem implants (ABI) in two patients with tinnitus has shown positive effects on tinnitus distress and loudness. ${ }^{9} \mathrm{We}$ however argue that the DCN is not the target of first choice for stimulation if tinnitus is long-standing. This is supported by a phenomenon called "progressive centralization". It has been suggested that chronically established tinnitus does not require the DCN for its expression. ${ }^{10}$ Over time, increased spontaneous activity in midbrain auditory nuclei becomes independent on afferent inputs, i.e. from DCN.11 Contemplating this intrinsically generated neuronal activity of upstream auditory nuclei and the undetermined rostral effects of DCN-HFS, a major effect of DCN stimulation on long-standing tinnitus is doubtful. Moreover, up till now ABI is only used in patients with severe hearing loss. Many tinnitus patients do not meet this criterium. Surgical reasons can also play a decisive role in target choice. From a neurosurgical perspective, the MGB is a more attractive target for DBS. From the vast amount of experience with thalamic DBS in the last two decades, we know that this region is readily accessible using stereotactic approaches, therefore expected to be associated with less surgical risks compared to DBS of deeper structures such as IC and DCN. The surgical approach required for DCN stimulation is known to be invasive and complex, making it less suitable for DBS. Novel techniques such as focused ultrasound ${ }^{12}$ and magnetothermal stimulation ${ }^{13}$ potentially allow neuromodulation of brain targets without invasive surgery being involved.

Regarding stimulation induced side-effects of DBS in auditory structures, hearing related symptoms are of particular concern. No evidence exists that DBS of the auditory pathway causes impaired hearing. Previously, it has been shown that DBS of the IC did not cause increased hearing thresholds as measured with $\mathrm{ABR}$ and sound induced prepulse inhibition. ${ }^{8}$ We confirmed that high frequency DBS of the DCN did not increase ABR thresholds. As 
stated above, results of ABR results should be interpreted cautiously. With ABR only a limited amount of frequencies is tested, recordings are prone to artefacts and responses provide no information on the auditory system above the brainstem level.

The limitations of assessing hearing using $\mathrm{ABR}$ are especially relevant for measurements during MGB stimulation, as the response of this nucleus in the $A B R$ is often not visible due to the stimulation artefact. Another potential sideeffect is the opposite, namely the generation of sounds. We have not been able to design or find a behavioral paradigm to assess the generation of sounds in our animal model. The function of the MGB is primarily hearing, which makes the occurrence of other side-effects than hearing-related effects unlikely. Considering the connections of the MGB with limbic regions and the proximity to motor nuclei of the thalamus, DBS of this nucleus might induce anxiety or locomotion related side-effects. These side-effects could both be positive or negative. We did not observe these side-effects with HFS as measured with Open Field and Elevated Zero Maze. Important to note is that stimulation induced side-effects are reversible. Nonetheless, whilst stimulating an auditory structure, hearing related side-effects are a potential risk and can, at this point, not be ruled out.

Side-effects due to current spread outside the MGB are unlikely to occur when the electrode is located in the center of the structure. The MGB is located posterior to the subthalamic nucleus and is $8 \mathrm{~mm}$ wide, $6 \mathrm{~mm}$ long and $6.5 \mathrm{~mm}$ high. Its volume is largest at $-3.5 \mathrm{~mm}$ from the AC-PC plane. Since neighboring structures of the MGB are structures which functional neurosurgeons are familiar with, we can speculate on which side-effects could occur in case current spread happens. Current spread to the anterior side of the MGB could result in internal capsule effects. These side-effects are known from STN DBS treatment for dystonia and Parkinson's disease. The possible side-effects of IC stimulation are dysarthria, muscle contractions, and gaze paresis. The ventricle can be found posterior from the MGB. Possible side-effects are known from DBS of the anterior nucleus of the thalamus for epilepsy which has also a border at the ventricle. Antero-medially the sensory thalamus is located which is targeted when performing DBS to treat pain. More medially the fields of Forel are located. Possible side effects are disturbances of speech, postural stability and gait. Lateral to the MGB the optic tract is located. We know from DBS of the globus pallidus interna that stimulation of the optic tract elicits phosphenes. All these side effects from current spread to surrounding regions are elicited by stimulation and thus reversible. 
Taking this together, a beneficial effect of DBS on tinnitus-like behavior was found in three structures within the auditory pathway: DCN, IC, and MGB. There were no indications that DBS induced side-effects such as hearing loss (DBS of DCN and IC), or anxiety and locomotion deficits (DBS of MGB). Small human studies have shown that tinnitus related distress can be alleviated by DBS of nonauditory structures as well. Our results point towards targeting the auditory circuit with DBS to successfully suppress tinnitus. In clinical setting, the MGB is the target of our first choice, given the vast amount of experience with thalamic DBS and that MGB-DBS encompasses the least surgical risks.

\section{The rationale for DBS from an electrophysiological perspective}

Considering the major societal impact of tinnitus, it is unfortunate how little is known about the exact neuropathology of tinnitus. To date most human tinnitus studies depend on non-invasive imaging and EEG studies. These noninvasive tools are limited by their temporal and spatial resolution. Several human studies show changes in volume, connectivity and activity both within and outside the classical auditory network. ${ }^{14-18}$ These studies show opposing results and are limited in their design by the heterogeneity of their symptoms. The advantage of using animal models is that measurements can be performed invasively, such that both a high temporal and spatial resolution can be achieved. Moreover, subjects are less heterogenic and possibilities exist to control for hearing loss and hyperacusis. With our electrophysiological studies, we aimed to gain more knowledge on the working mechanisms of DBS, and also the pathophysiology of tinnitus, which is of importance for clinical application of such therapies.

Our electrophysiology study described in chapter 5 confirmed spontaneous hyperactivity in the MGB as a neural correlate of tinnitus. Specifically, sustained and suppressed response type exhibited enhanced spontaneous activity, while fast responding neurons became less- or non-responsive. In the context of the central gain model, it is tempting to speculate that the loss of fast-responding neurons is causally linked to the increased spontaneous firing of the other two response types. Even though we could not find a correlation, causality cannot completely be ruled out. The study described in chapter 6 , showed that noise-exposed animals could not distinguish temporal regularity in auditory stimuli. This inability to distinguish regular and irregular timing 
can indicate that sensory gating is disturbed. Tinnitus has previously been associated with predictive coding. ${ }^{19-21}$ Support for dysfunctional sensory gating mechanisms in the thalamus in persons with tinnitus was recently further supported in a in a graph-theoretical study. ${ }^{22}$ The altered firing patterns might modulate the gating function of the thalamus and thereby result in maladaptive gating.

The question on how DBS acts on aberrant neuronal tinnitus activity and gating mechanism remains. Working mechanisms of low frequency stimulation (LFS) and HFS are probably dissimilar.

In our studies, the positive effects on tinnitus behavior were observed during HFS of the DCN, IC and MGB. Interestingly, low frequency stimulation of the MGB did not have a beneficial effect on tinnitus-like behavior. Previously, it has been shown that electrical stimuli with auditory brainstem implants (ABI) with a high frequency $(250-4000 \mathrm{~Hz})$ can lead to changes in tinnitus loudness. ${ }^{23}$ These stimuli differ from continuous HFS by DBS, as the former are noncontinuously presented analogues of acoustic waveforms to restore hearing. Both ABI-stimulation and LFS can have excitatory effects.

If the DCN of someone with severe hearing loss is stimulated with ABI, a partial restoration of reduced auditory input is established. LFS of the auditory tract can have a similar effect, as one small preclinical study finds a suppressing effect of LFS on short term tinnitus when applied on the DCN in rats. ${ }^{24}$ These effects can be compared to the effect of hearing aids and cochlear implants to inhibit tinnitus. The effect of HFS cannot be explained by a restoration of the lack of input. First, we did not find an increase in c-Fos expression after HFS DCN stimulation in the IC, MGB and A1. And second, electrophysiological measurements did not show an increase in power and synchronicity after HFS. More likely, HFS disrupts aberrant patterns of activity or temporal coherence, eventually resulting in restoration of information flow. It is known that conventional HFS DBS leads to a relative inactivation of the neurons around the stimulation electrode. ${ }^{25}$ This is because HFS stimulation induced action potentials take the lead, which causes neurons to lose the ability to transit information. We postulate that this informational lesion within a neural network disorder, such as in tinnitus, can eliminate pathological oscillations, normalize neural firing patterns and potentially restore sensory gating in the MGB. 
Our results in chapter 6 indeed confirmed a temporary suppression of thalamocortical synchronicity in beta and gamma frequency bands after MGB DBS. It has been suggested that tinnitus is associated with enhanced thetagamma coupling, ${ }^{26}$ causing thalamocortical dysrhythmia. Our findings support that MGB-DBS has potential to disrupt this aberrant activity. Despite that we found some evidence for disturbed sensory gating in tinnitus, we did not find evidence that MGB DBS restores the sensory gating capacity in the noiseinduced tinnitus animal model.

The results from our electrophysiology studies support multiple existing theories on DBS and tinnitus pathophysiology, which strengthens our hypothesis that DBS ultimately will be effective to treat humans suffering from tinnitus. Neural plasticity is likely one of the involved mechanisms of DBS, as we found a residual effect of both HFS DBS on tinnitus-like behavior, and thalamocortical desynchronization. Further studies are needed to determine the exact duration of this remaining behavioral effect. This residual effect is of interest for potential clinical practice, as stimulation in ON-OFF cycles can be considered, which saves battery life and potentially reduces side- and habituation effects.

\section{Future perspectives}

The increasing evidence that tinnitus can be alleviated by modulation of central auditory targets is promising. Still, extreme caution has to be taken, in order to safely and effectively translate these preclinical findings to clinical practice. As discussed, it is uncertain how the preclinical noise-exposed tinnitus model represents human tinnitus. Results might only be extrapolated to a subset of tinnitus patients, presumably patients with unilateral pure-tone tinnitus. Species may perceive sounds differently. Humans relate sounds to meaning and memories, and this may be dissimilar in rats. We suppose that GPIAS results represent the loudness of a tinnitus sound. As we know from human research, this does not necessarily correspond to tinnitus severity. Up till now, no reliable behavioral correlate of tinnitus has been found in humans. Gap detection testing does not seem to be a valid measure in humans, ${ }^{27,28}$ possibly because cortical processing in humans compensates for minor subcortical deficits. Thus, tinnitus in human may be much more complex than the existing animal models. 
At the moment, modulation of central auditory structures can easiest be achieved with DBS. Neurosurgeons consider DBS as a minimally invasive approach. Still, other surgical or non-invasive neuromodulation techniques should not be neglected. It is to be expected that in the next decades, non or at least even less-invasive approaches to modulate central neuronal activity will become available.

The logical next step is to conduct a clinical pilot trial on the effect of DBS in severe and chronic tinnitus patients. Our target of first choice for this approach is the MGB. Remaining questions on how to define the optimal target and stimulation settings remain and may only be answered in a larger phase II trial. Human tinnitus studies are currently limited by their low temporal resolution (using MRI) and spatial resolution (using EEG). Performing this clinical study on MGB DBS creates the unique opportunity to acquire neural activity direct from the MGB both intra-operatively from the micro-electrode recordings and postoperatively from the DBS electrodes. Such valuable data could help to gain further insight in the underlying neurophysiological alterations which causes tinnitus. These findings could help establishing new concepts in the development of effective treatment options for persons with tinnitus. 


\section{References}

1. Smit JV, et al. Hearing assessment during deep brain stimulation of the central nucleus of the inferior colliculus and dentate cerebellar nucleus in rat. Peer J. 2017;5:e3892.

2. Shi Y, et al. Deep brain stimulation effects in patients with tinnitus. Otolaryngol Head Neck Surg. 2009;141(2):285-7.

3. Smit JV, et al. The impact of deep brain stimulation on tinnitus. Surg Neurol Int. 2016;7(Suppl 35):S848-S854.

4. Cheung SW, Larson PS. Tinnitus modulation by deep brain stimulation in locus of caudate neurons (area LC). Neuroscience. 2010;169(4):1768-1778.

5. Cheung SW, et al. Phase I trial of caudate deep brain stimulation for treatment-resistant tinnitus. J Neurosurg. 2019:1-10.

6. Dijkstra E, et al. Effective deep brain stimulation of intractable tinnitus: A case study. Brain Stimul. 2018;11(5):1205-1207.

7. Jakobs M, Lozano AM. Editorial. Deep brain stimulation for tinnitus: exploring the frontier between sensory perception and awareness. J Neurosurg. 2019:1-4.

8. Smit JV, et al. Deep brain stimulation of the inferior colliculus in the rodent suppresses tinnitus. Brain Res. 2016;1650:118-124.

9. van den Berge MJC. Neurosurgical interventions at the cochlear nerve \& nucleus for treatment of tinnitus. 2020.

10. Brozoski TJ, Bauer CA. The effect of dorsal cochlear nucleus ablation on tinnitus in rats. Hear Res. 2005;206(1-2):227-236.

11. Mulders WH, Robertson D. Progressive centralization of midbrain hyperactivity after acoustic trauma. Neuroscience. 2011;192:753-760.

12. Lipsman $\mathrm{N}$, et al. MR-guided focused ultrasound thalamotomy for essential tremor: a proofof-concept study. Lancet Neurol. 2013;12(5):462-468.

13. Chen R, et al. Wireless magnetothermal deep brain stimulation. Science. 2015;347(6229): 1477-1480.

14. Muhlau M, et al. Structural brain changes in tinnitus. Cereb Cortex. 2006;16(9):1283-1288.

15. Allan TW, et al. Neuroanatomical Alterations in Tinnitus Assessed with Magnetic Resonance Imaging. Front Aging Neurosci. 2016;8:221.

16. Chen $\mathrm{YC}$, et al. Aberrant spontaneous brain activity in chronic tinnitus patients revealed by resting-state functional MRI. Neuroimage Clin. 2014;6:222-228.

17. Hofmeier B, et al. Reduced sound-evoked and resting-state BOLD fMRI connectivity in tinnitus. Neuroimage Clin. 2018;20:637-649.

18. Lv H, et al. Altered functional connectivity of the thalamus in tinnitus patients is correlated with symptom alleviation after sound therapy. Brain Imaging Behav. 2020;14(6):2668-2678.

19. De Ridder D, et al. Thalamocortical Dysrhythmia: A Theoretical Update in Tinnitus. Front Neurol. 2015;6:124.

20. Hullfish J, Sedley W, Vanneste S. Prediction and perception: Insights for (and from) tinnitus. Neurosci Biobehav Rev. 2019;102:1-12.

21. Sedley W, et al. An Integrative Tinnitus Model Based on Sensory Precision. Trends Neurosci. 2016;39(12):799-812.

22. Lin X, et al. Altered Topological Patterns of Gray Matter Networks in Tinnitus: A GraphTheoretical-Based Study. Front Neurosci. 2020;14:541.

23. Soussi T, Otto SR. Effects of electrical brainstem stimulation on tinnitus. Acta Otolaryngol. 1994;114(2):135-140.

24. Luo H, et al. Tinnitus suppression by electrical stimulation of the rat dorsal cochlear nucleus. Neurosci Lett. 2012;522(1):16-20.

25. Lozano AM, et al. Deep brain stimulation: current challenges and future directions. Nat Rev Neurol. 2019;15(3):148-160. 
26. Vianney-Rodrigues $\mathrm{P}$, Auerbach BD, Salvi R. Aberrant thalamocortical coherence in an animal model of tinnitus. J Neurophysiol. 2019;121(3):893-907.

27. Fournier P, Hebert S. Gap detection deficits in humans with tinnitus as assessed with the acoustic startle paradigm: does tinnitus fill in the gap? Hear Res. 2013;295:16-23.

28. Boyen K, Baskent D, van Dijk P. The Gap Detection Test: Can It Be Used to Diagnose Tinnitus? Ear Hear. 2015;36(4):e138-145. 


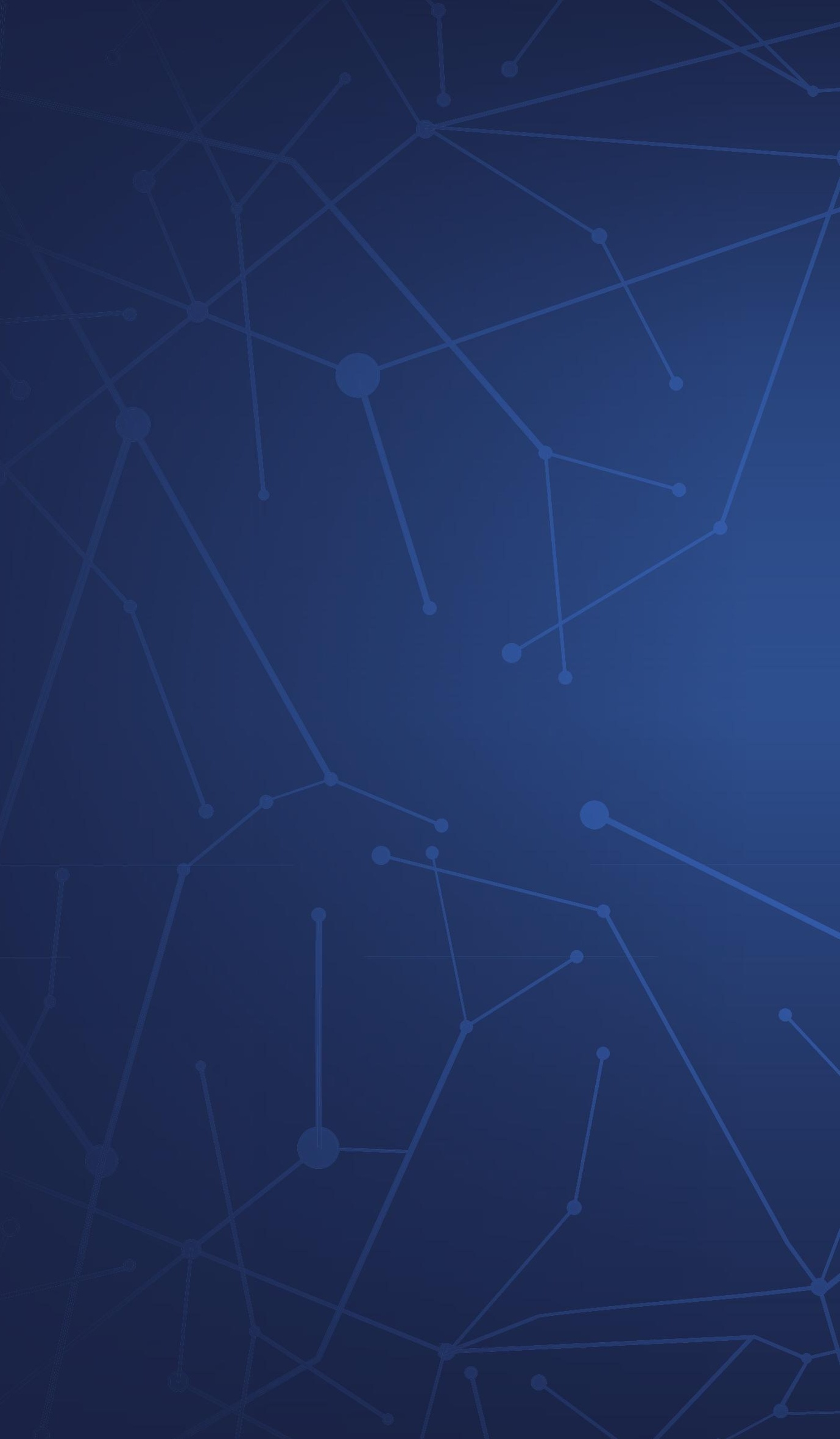


ADDEND出 

Summary 



\section{Summary}

The studies presented in this thesis examined the efficacy of deep brain stimulation (DBS) in central auditory structures on tinnitus suppression in preclinical setting (Part I), as well as neural mechanisms underlying tinnitus pathophysiology and DBS (Part II). These findings are translated from bench to bedside in a protocol for a first-in-human clinical pilot study on DBS in severe, refractory tinnitus (Part III).

\section{Part I}

Chapter 1 describes current developments in the field of neuromodulation and specifically DBS for tinnitus suppression. There is growing evidence that DBS might be able to interrupt altered neuronal activity within the complex tinnitus network and herewith suppress tinnitus. The dorsal cochlear nucleus (DCN), inferior colliculus (IC) and medial geniculate body of the thalamus (MGB) are auditory structures that show increased spontaneous firing and synchronicity in tinnitus, which make it candidate targets for DBS. However, preclinical evidence is currently lacking and required to support or discourage clinical application of DBS in tinnitus.

Following this, Chapter 2 assesses the effect of bilateral high frequency stimulation (HFS) of the DCN on noise-induced tinnitus in rats. After noise trauma, tinnitus was confirmed with gap-prepulse inhibition of the acoustic startle response paradigm (GPIAS), whilst contralateral hearing was preserved as measured with Auditory brainstem responses (ABR). Behavioral testing showed tinnitus suppression during HFS. HFS did not influence hearing thresholds. These results are in line with a previous study within our group on DBS of the IC. Regarding DBS mechanisms, HFS did not change c-FOS expression in the central auditory pathway. This implies that HFS does not simply stimulates or inhibits neuronal activity, but rather influences firing patterns and synchronicity within the pathophysiological tinnitus network.

Chapter 3 involves a methodologically similar preclinical study in which the efficacy of MGB-DBS is evaluated. Here, we found a direct and residual suppressing effect on tinnitus during HFS. On the contrary, low frequency stimulation (LFS) did not suppress tinnitus behavior. No anxiety or locomotor related side-effects were observed during HFS, as measured with the elevated 
zero maze and open field test. We postulated that HFS indeed causes an information lesion by eliminating pathological oscillations, which is dissimilar to the effect of LFS which is known to stimulate neuronal activity. This study confirms the prominent role of the MGB in tinnitus pathophysiology. Yet, available literature is sparse and only a handful of papers have been published on this matter.

\section{Part II}

In Chapter 4 we first aimed to characterize electrophysiological hallmarks in the MGB after noise-exposure. In total, we recorded activity of 214 single units in noise-exposed tinnitus animals and unexposed controls. We classified all recorded neurons into four response types: fast, sustained, suppressed and no responses. Novel findings suggest a distinct role for separate functional classes of neurons after noise trauma. Fast responding neurons become less or nonresponsive without a change to the spontaneous firing rate. We hypothesized that these neurons are mainly involved in hearing loss mechanisms. Sustained and suppressed responding neurons exhibited increased spontaneous firing, without a change to the stimulus driven activity. Possibly, this increased activity is responsible for phantom perception in tinnitus. In the second part of this chapter we present the effect of HFS on spontaneous local field potentials in the MGB. Our findings show that HFS of the MGB suppressed thalamocortical synchronization in beta and gamma frequency bands. In the light of thalamocortical dysrhythmia in tinnitus, we proposed that desynchronization is an underlying mechanism of tinnitus suppression by MGB DBS.

Chapter 5 explores the role of the MGB in sensory gating in noise-exposed and control subjects before and directly after MGB DBS. We found first evidence that thalamic filtering occurs at the level of the MGB in rats, concerning stimulus pitch, position and regularity. Noise-exposure abolished the effect of temporal regularity on evoked potentials' amplitudes, indicating that sensory gating is reduced in noise-exposed tinnitus animals. Furthermore, evoked potentials were overall increased after noise-trauma, possibly because of increased synchronous firing. We did not find evidence that DBS restored sensory gating function. Still, DBS led to a significant overall decrease in evoked potentials, which was specific to the noise-exposed group. 


\section{Part III}

The first step in translating our findings to clinical practice is the protocol for a first-in-human pilot trial, as presented in Chapter 6. Based on current preclinical evidence and available literature, targeting subcortical auditory structures with DBS seems a promising approach. Taking surgical risks into account, the MGB became the target of our first choice, as this nucleus is best accessible using stereotaxy. The chapter describes a protocol for a double-blind crossover clinical pilot trial on DBS of the MGB in six patients with severe, refractory tinnitus. The primary focus of this study is to evaluate safety and feasibility (acceptability). Secondary objectives are tinnitus severity as measured with the Tinnitus Functional Index (TFI), tinnitus loudness and distress, hearing, cognitive and psychological functions, quality of life and neurophysiological characteristics.

Besides recommendations for clinical practice, the general discussion in Chapter 7 addresses our preclinical setup and its limitations, the question which target is preferred for DBS in tinnitus and reflects on what has been learned from our electrophysiological studies. 

Nederlandse samenvatting 



\section{Samenvatting}

De studies in dit proefschrift richten zich op de effectiviteit van diepe hersenstimulatie (DBS) in centrale auditieve structuren op tinnitusonderdrukking in een preklinische setting (Deel I), evenals centrale mechanismen die ten grondslag liggen aan tinnitus en DBS (Deel II). Resultaten van het onderzoek worden vertaald van bench to bedside in een protocol voor een eerste klinische pilotstudie naar DBS bij patiënten met ernstige, refractaire tinnitus (Deel III).

\section{Deel I}

Hoofdstuk 1 geeft een overzicht van de huidige ontwikkelingen op het gebied van neuromodulatie en specifiek DBS voor de behandeling van tinnitus. Het beschrijft aanwijzingen dat DBS abnormale neuronale activiteit in een complex netwerk kan onderbreken en op deze manier tinnitus zou kunnen verminderen. De dorsale cochleaire kern (DCN), colliculus inferior (IC) en corpus geniculatum mediale van de thalamus (MGB) zijn drie auditieve structuren die een verhoogde spontane neuronale vuurfrequentie en synchroniciteit vertonen in tinnitus. Dit maakt deze structuren veelbelovend voor toepassing van DBS om tinnitus te onderdrukken. Tot op heden ontbreekt voldoende preklinisch bewijs om een klinische toepassing van DBS te ondersteunen of juist te ontmoedigen.

Hoofdstuk 2 beschrijft het onderzoek naar het effect van bilaterale hoogfrequente stimulatie (HFS) van de DCN op lawaai-geïnduceerde tinnitus bij ratten. Na geluidstrauma werd getest of de dieren tinnitus hadden, met een hiervoor ontwikkeld startle-response paradigma, genaamd gap prepulse inhibition of the acoustic startle (GPIAS). Ook werd gecontroleerd of het gehoor aan de contralaterale zijde behouden bleef, door het meten van auditieve hersenstam responsen. Gedragstesten gaven aan dat tinnitus onderdrukt kon worden met HFS, zonder meetbaar effect op de gehoordrempel. Deze resultaten komen overeen met die van een eerdere studie van onze groep over DBS van de IC. HFS had geen effect op de expressie van c-FOS in de centrale auditieve kernen. Dit impliceert dat HFS niet simpelweg neuronale activiteit stimuleert of remt, maar eerder neuronale vuurpatronen en synchroniciteit beïnvloedt binnen het pathofysiologische tinnitus netwerk. 
Hoofdstuk 3 betreft een methodologisch vergelijkbare preklinische studie waarin de werkzaamheid van DBS van de MGB wordt geëvalueerd. Ook in deze studie vonden we een positief effect van HFS in ons tinnitus-model. Dit effect is meetbaar gedurende ten minste enkele minuten na stimulatie. In tegenstelling tot HFS had laagfrequente stimulatie (LFS) geen effect op tinnitusgedrag. Metingen met de elevated zero maze en open field test lieten geen angst- of beweging gerelateerde bijwerkingen zien tijdens HFS. De bevinding dat specifiek HFS, en níet LFS, van meerdere structuren in het auditieve systeem tinnitus kan verminderen, pleit voor de hypothese dat HFS pathologische oscillaties in het tinnitus netwerk kan doorbreken. De werking van HFS is anders dan van LFS, waarvan bekend is dat de laatste juist neuronale activiteit stimuleert. Deze studie bevestigt de prominente rol van de MGB in de pathofysiologie van tinnitus. De beschikbare literatuur is schaars, er zijn slechts een handvol artikelen over dit onderwerp gepubliceerd.

\section{Deel II}

In Hoofdstuk 4 hebben we ons eerst gericht op de invloed van geluidstrauma op de neurofysiologische karakteristieken in de MGB. In totaal hebben we de activiteit van 214 afzonderlijke neuronen gemeten in zowel ratten die blootgesteld waren aan geluidstrauma als ook gezonde controles. Op basis van de reactie van de neuronen op geluiden, classificeerden we de responsen in vier typen: snelle, persisterende, onderdrukte of afwezige responsen. Onze bevindingen suggereren een verschillende rol voor afzonderlijke functionele klassen van neuronen in relatie tot geluidstrauma. Het blijkt dat snel reagerende neuronen minder of niet responsief worden, zonder dat de spontane vuurfrequentie verandert. We stelden dat deze neuronen voornamelijk betrokken zijn bij mechanismen voor gehoorverlies. Zowel persisterende als onderdrukt reagerende neuronen vertoonden een spontane overactiviteit na geluidstrauma, zonder dat de stimulus-gedreven activiteit veranderde. Mogelijk zijn deze neuronen verantwoordelijk voor fantoomperceptie bij tinnitus. In het tweede deel van dit hoofdstuk presenteren we het effect van HFS op spontane local field potentials in de MGB. Specifiek keken we naar de synchroniciteit tussen de thalamus en cortex, welke in tinnitus verstoord kan zijn. De resultaten tonen aan dat HFS van de MGB deze thalamocorticale synchronisatie onderdrukt, specifiek in bèta en gamma frequentiebanden. Op basis van deze bevindingen suggereren wij dat 
thalamocorticale desynchronizatie een onderliggend mechanisme kan zijn van tinnitus onderdrukking door MGB DBS.

Hoofdstuk 5 beschrijft het onderzoek naar de rol van de MGB in sensory gating en wat de invloed is van geluidstrauma en DBS. We vonden de eerste aanwijzingen dat auditieve filtering optreedt op het niveau van de MGB in ratten, op basis van variatie van de toonhoogte, positie en regelmatigheid van auditieve stimuli. We vonden aanwijzingen dat sensory gating verminderd was in de dieren die waren blootgesteld aan geluidstrauma, aangezien er geen verschil was in de amplitudes van de evoked potentials bij verschillen in temporele regelmaat. Ook waren evoked potentials verhoogd na geluidstrauma, wat mogelijk veroorzaakt wordt door verhoogd synchroon vuren van neuronen. Wij vonden geen bewijs dat DBS de sensory gating functie herstelde. Toch leidde DBS tot een significante afname van de verhoogde amplitudes van de evoked potentials, wat specifiek was voor de aan geluidstrauma blootgestelde diergroep.

\section{Deel III}

De eerste stap om onze bevindingen te vertalen naar een klinische toepassing is een first-in-human pilotstudie, waarvan het protocol is beschreven in Hoofdstuk 6. Het huidige preklinische onderzoek naar de werkzaamheid van DBS bij tinnitus en de literatuur hierover, wijst in de richting van de MGB als hersenstructuur van eerste keuze voor klinische DBS bij ernstige tinnitus. Het protocol beschrijft een dubbelblinde cross-over pilotstudie naar DBS van de MGB bij zes patiënten met ernstige, therapieresistente tinnitus. Het belangrijkste doel van deze studie is het evalueren van de veiligheid en uitvoerbaarheid van de behandeling. Secundaire uitkomsten zijn de ernst van tinnitus (gemeten met Tinnitus Functional Index, (TFI)), tinnitus luidheid en last (gemeten middels visueel analoge schaal, (VAS)), gehoor, kwaliteit van leven en cognitief en psychologisch functioneren. Bovendien worden neurofysiologische metingen verricht, wat een unieke mogelijkheid is om meer inzichten te krijgen in de functie van de MGB.

Naast aanbevelingen voor de klinische praktijk, gaat de algemene discussie in Hoofdstuk 7 in op onze preklinische opzet en de beperkingen daarvan, op de vraag welke hersenstructuur de voorkeur heeft voor DBS bij tinnitus en 
reflecteren we op de inzichten die we gekregen hebben van onze elektrofysiologische studies. 
Impact paragraph 



\section{Impact paragraph}

Tinnitus is one of the major health care problems of the current era, as it poses a high burden on individuals as well as the whole society. It is expected that $2.4 \%$ of the general population suffers from the most severe degree of tinnitus, which is accompanied by a wide range of psychological symptoms such as irritability, insomnia, anxiety and depression.1,2 Tinnitus-related costs for society are substantial, with a total estimated amount of $€ 6.8$ billion per year in the Netherlands, of which the main part is not health care related. ${ }^{3}$ The disease specific health care costs account for $2.3 \%$ of the total health care expenditure. ${ }^{3}$ These numbers illustrate that we are still in need of an effective therapy. So far, therapies have aimed at symptomatic relief, but fail to eliminate the tinnitus percept. ${ }^{4}$ Deep brain stimulation (DBS) has been put forward as a potential treatment in severe tinnitus. ${ }^{5}$ However, sufficient knowledge on neuropathophysiology of tinnitus and potential working mechanisms of DBS is still lacking, which hinders development of this therapy in severe tinnitus cases. With the preclinical studies presented in this thesis, we aimed to investigate the potential of DBS of structures within the auditory pathway to suppress tinnitus and to gain knowledge on potential neurophysiological mechanisms with regard to tinnitus and DBS. This way, we intended to help forming a solid scientific base which is needed in order to apply DBS in a clinical setting.

The main finding of this thesis is that high frequency stimulation of multiple structures within the central auditory pathway can suppress tinnitus. Additionally, our findings support the key role of the medial geniculate body (MGB) in tinnitus pathophysiology and suggest desynchronization of thalamocortical oscillations as an underlying mechanism of DBS.

\section{Target audience}

First and foremost, the work of this thesis is eventually relevant for patients and their relatives. An effective therapy for severe and refractory tinnitus is currently an unmet medical need, leading to a poor quality of life in millions of people worldwide. ${ }^{4}$ Unfortunately, tremendous efforts in the past decades have not led to breakthroughs in the search for curative tinnitus treatments. DBS has the potential to interact with the actual core of the tinnitus network, which is a novel and therefore promising therapeutic approach. Hopefully, the findings in this thesis contribute to the development of DBS and ultimately 
other types of non-invasive neuromodulation to suppress tinnitus, so that patients will benefit.

Once patients with severe, disabling tinnitus can function more independently, the general population will benefit as well. Effective treatment of severely affected tinnitus patients will be a financial relief for society, as less healthcare related costs will be made, while productivity benefits will increase as patients are able to work, consume and invest more.

Before this happens, the findings should be evaluated and incorporated by the next important target group: the academic community. The results of the behavioral studies on target choice, tinnitus suppression, side-effects, and stimulation parameters are relevant to a multi-disciplinary group of clinicians and researchers, such as neurosurgeons, ENT physicians, neurologists, audiologists and psychiatrists. In my view, a basic understanding of the complexity of a symptom such as tinnitus, as shown in this thesis, is also beneficial for general practitioners who deal on a daily basis with patients with scientifically poorly understood chronic conditions. With this background knowledge, it is possible to understand the heterogeneity of symptoms and patient presentations and can ultimately help showing compassion, which has many benefits on itself. Lastly, mechanistic findings underlying tinnitus pathophysiology and DBS effects are relevant to researchers, but also neuromodulation companies. Huge advances are being made in the field of neuromodulation. Besides optimizing DBS therapy, other non-invasive techniques are being developed that will potentially be used in auditory structures to suppress tinnitus as well. Examples are focused ultrasound or wireless magnetothermal deep brain stimulation to modulate neuronal activity. ${ }^{6,7}$

\section{Innovation}

The work described in this dissertation can be considered innovative in several ways. The first reason is that we used a schematic translational approach for this research line. This of course is not new on itself, but it is different compared to most previous established indications for DBS. Most indications for DBS derive from coincidental clinical findings which were subsequently directly applied in humans, often without a solid scientific base. With the evidence from our translational research line we aim to improve effectiveness and efficiency of our clinical studies. Second, we tried to shift the therapeutic focus of tinnitus. While current therapies have mostly focused on comorbidities 
of tinnitus in order to improve quality of life, we try and focus on the center of the problem and aim to eliminate the phantom sound by modulation of central auditory neuronal activity. Third, our findings put the MGB forward as a central key structure, a target that so far has been given just little attention in scientific tinnitus literature. As far as we are aware, we are the first to show an effect of MGB DBS on tinnitus behavior. We have shown novel findings that the MGB indeed serves as a filtering station for auditory stimulus processing, which is functioning differently in tinnitus animals. Furthermore, our explorative electrophysiological study points towards distinct roles of multiple functional classes of neurons in tinnitus pathophysiology, which need to be further investigated.

\section{Implementation}

The novel insights gained from this thesis will be implemented in various ways. Knowledge has been and will be shared in peer-reviewed international journals and during national and international conferences. On the short term, the results from the first part of this thesis lead to the clinical pilot study on MGB DBS in severe refractory tinnitus, which is currently being carried out at Maastricht UMC+. With this first-in-human study, Maastricht UMC+ is in the front line of DBS research. This clinical investigation of a novel indication for DBS impacts our international leading position in the field. With the exploratory studies of the second part of this dissertation, we encourage scientists to continue research on these electrophysiological findings. We hope that especially the MGB will be given more attention in future mechanistic and therapeutic research of tinnitus, as evidence shows promising characteristics of this structure for neuromodulative approaches. Furthermore, the clinical pilot study protocol that is presented in the third part provides a unique opportunity to record local field potentials within the human MGB. The procedure for performing these recordings will be based on the recording protocol that we developed for our preclinical study. On the longer term, welldesigned studies with an adequate sample size should investigate therapeutic effects of DBS and ultimately other non-invasive neuromodulative techniques. Eventually, we should aim for individually optimized treatments, based on combined data such as each patient's symptom characteristics, neurophysiological recordings and fMRI data. 


\section{References}

1. Axelsson A, Ringdahl A. Tinnitus--a study of its prevalence and characteristics. Br J Audiol. 1989;23(1):53-62.

2. Henry JA, Dennis KC, Schechter MA. General review of tinnitus: prevalence, mechanisms, effects, and management. J Speech Lang Hear Res. 2005;48(5):1204-1235.

3. Maes IH, Cima RF, Vlaeyen JW, Anteunis LJ, Joore MA. Tinnitus: a cost study. Ear Hear. 2013;34(4):508-514.

4. Langguth B, Kreuzer PM, Kleinjung T, De Ridder D. Tinnitus: causes and clinical management. Lancet Neurol. 2013;12(9):920-930.

5. Smit JV, Janssen ML, Schulze H, et al. Deep brain stimulation in tinnitus: current and future perspectives. Brain Res. 2015;1608:51-65.

6. Martin E, Jeanmonod D, Morel A, Zadicario E, Werner B. High-intensity focused ultrasound for noninvasive functional neurosurgery. Ann Neurol. 2009;66(6):858-861.

7. Roet M, Hescham SA, Jahanshahi A, Rutten BPF, Anikeeva PO, Temel Y. Progress in neuromodulation of the brain: A role for magnetic nanoparticles? Prog Neurobiol. 2019;177: $1-14$. 
Dankwoord 



\section{Dankwoord}

Zelf kan ik het eigenlijk nog niet geloven, maar mijn proefschrift is nu toch echt helemaal af. Dit was nooit gelukt zonder de hulp en steun van velen. Graag wil ik mijn promotieteam en een aantal mensen in het bijzonder bedanken.

Prof. Temel, beste Yasin, bedankt dat je mij het vertrouwen hebt gegeven om binnen het Neuroscience lab dit translationele promotietraject uit te voeren. Je enthousiasme en passie voor wetenschappelijk onderzoek hebben mij altijd erg geïnspireerd en zijn een drijvende kracht geweest achter dit promotietraject. De combinatie van je positiviteit en kritische blik werkten erg motiverend om nieuwe hypothesen te vormen en vervolgens te testen. Bedankt!

Prof. Stokroos, beste Robert, als student kwam ik op gesprek om de mogelijkheden voor een wetenschapsstage in het buitenland te bespreken. De ervaring die ik mede dankzij jou in Canada heb kunnen opdoen was fantastisch en heeft op vele manieren invloed gehad op mijn verdere loopbaan. Het was een bijzondere eer dat je mij daarna de mogelijkheid gaf om dit uitdagende promotietraject te starten, waar al veel voorbereidend werk aan vooraf was gegaan. Bedankt voor de vrijheid die je me hebt gegeven om keuzes te maken die bij mij passen, dit heb ik enorm gewaardeerd.

Dr. Janssen, beste Mark, jouw energie en besluitvaardigheid hebben dit onderzoek op een moment van stagnatie opnieuw in een stroomversnelling gebracht! Ik ben je ontzettend dankbaar voor de vele uren - vaak 's avonds na de normale werkzaamheden - die we samen aan alle projecten hebben gewerkt. Met jou als copromotor had ik een enorm luxeprobleem, omdat ik door de snelle correcties op manuscripten nooit iets uit mijn hoofd kon zetten. Bedankt, zonder jou was dit proefschrift nooit geworden wat het nu is.

Beste leden van de beoordelingscommissie, hartelijk dank dat jullie de tijd en moeite hebben willen nemen om mijn proefschrift te evalueren.

Naast mijn promotieteam wil ik nog een aantal mensen bedanken voor hun onmisbare bijdragen aan het onderzoek. Allereerst Dr. Smit, Jasper, dankzij jou kwam ik in een gespreid bed terecht en kende mijn promotietraject een vliegende start. Je leerde me ongeveer alles wat bij proefdieronderzoek komt kijken en gaf me perspectief als ik tijdens een experiment na weken zonder 
daglicht weer eens gepikeerd uit de kelder kwam. Ik ben ontzettend blij dat je ook het klinische project verder getrokken hebt en er mooie studies in het verschiet liggen.

Dr. Roberts, dear Mark, it is hard to express how much I learned from you. Your patience and programming skills are of the highest level I ever experienced. Even though it must have been frustrating at times to work with someone without a Matlab background ánd a MacBook, you never showed it! I owe you a huge thanks for working with me on the chapter I am most proud of.

Dr. Jahanshahi, dear Ali, thank you for always being helpful. Your expertise brought the project to a higher level for which I am very grateful. You taught me how to act adequately in acute situations and I am glad I now master the skill of rat-resuscitation.

Prof. Kotz, Dr. Schwartze, dear Sonja and Michael, thank you for our exciting collaboration, which started as a "side project", but quickly grew after the first results and interesting discussions we had. I am looking forward to see what the future studies will bring.

All other coauthors, thank you for the fruitful collaborations, I learned a lot from your input and help.

Lieve paranimfen, het betekent veel voor me dat jullie op deze belangrijke dag aan mijn zijde staan. Anne, jouw humor en onze acties hebben me echt door alle fasen van het promotietraject gesleept. De herinneringen aan een groot aantal hilarische momenten brengen nog vaak een lach op mijn gezicht. Hoewel jij zelf niet aan spiegelen deed, houd je wel vaak een spiegel voor me tijdens fijne gesprekken. Bedankt voor onze vriendschap! Lieve Ruth, dankzij ons co-ouderschap is onze band extra speciaal. De talloze avonturen die wij samen beleefd hebben waren altijd de perfecte manier van ontspanning! Van beklimmingen tot grote hoogte op mooie bergen in heel Europa, tot diepe duiken in Cyprus, niets is jou te gek. Recent heb je me ook nog aangestoken met het kitesurf virus, alsof ik nog niet genoeg hobby's had. Ik hoop dat er nog vele belevenissen zullen volgen, maar daar twijfel ik niet aan.

Dear colleagues of the Neuroscience lab, thanks to you my time in the lab was amazing and more memorable than I ever expected it to be. Fréderic met $\mathrm{c}$, lieve Fred, vanaf het moment dat we bij elkaar in hetzelfde kantoor waren werden we al snel een goed team. Jij was altijd hard aan het klikken, terwijl ik luid zat te tikken. Ons congres in San Diego en de reis nadien waren geweldig. 
Het surfen, de hele grote boom en rupsen in Death Valley zal ik nooit vergeten. Ook in moeilijkere tijden heb ik veel steun aan je gehad, dankjewel! Milaine, wat was het fijn om jou als nuchtere Haagse in ons midden te hebben. Ik kijk terug op mooie momenten, zoals in Tijuana, en heb van de tijd samen genoten (behalve als je weer eens aan het zagen en timmeren was). Mijn directe collega's en mede-promovendi, Sarah, Sandra, Birgit, Paul, Majed, Jeroen, Margot, Sylvana, Lianci en Faris, bedankt voor de hulp en de gezellige tijd. Onze dag tussen de Alpaca's was voor mij een hoogtepunt. Jana, bedankt dat jij het project voortzet! Aryo, thanks for your help and the time you have put in the analyses in one of our projects, I wish you the best of luck in Germany. Overige collega's van Divisie 3, wat ben ik blij dat ik jullie heb leren kennen. Artemis, your "gezelligheid" and cooking skills are extra-ordinary, thanks for the great times we shared! Marina, Koen, Glenn, Roel, Nynke, Perla and other colleagues, thanks for the interesting lunchbreaks and memorable lab days out. Marlieke, de vele uren die je achter de microscoop hebt doorgebracht hebben zeker zijn vruchten afgeworpen, bedankt! Hellen, Denise en Marjan, de onmisbare kern van het Neuroscience lab, bedankt voor jullie hulp bij al mijn experimenten. Ook wil ik graag alle medewerkers van het proefdierlab bedanken voor hun assistentie.

Collega's van de KNO en audiologie, bedankt voor de gezellige en leerzame momenten. Remo, Elke, Marc en Tim, onze cochlea-tot-cortex discussies kan ik me nog levendig voor de geest halen en lieten me inzien wat voor complex project ik nou eigenlijk begonnen was. Floor, bedankt voor de talloze fijne avondjes en alle gedeelde sushi rollen!

Alle medewerkers van Medisch Centrum de Linde en Medisch Centrum Margraten, en in het bijzonder mijn opleiders Chris Hanssen, Gerard Priem en Paulien Schunck: bedankt voor jullie flexibiliteit, jullie interesse in mij als persoon en in mijn onderzoek. De fijne tijd die ik als huisarts in opleiding bij jullie had maakte het zoveel makkelijker om in overige tijd aan mijn onderzoek te werken.

Vele lieve vrienden hebben me over de jaren bijgestaan. Lisa, Miranda, Mirjana en Kim, lieve Moeders, wij kwamen als Noordelijke lotgenoten bij elkaar toen we allemaal per ongeluk in Maastricht werden ingeloot. Lief en leed hebben we gedeeld en ik ben dankbaar dat onze vriendschap nog steeds zo hecht is, ondanks dat we ons intussen allemaal naar een andere uithoek van Nederland 
hebben verplaatst. Bedankt voor onze vele weekendjes, wijntjes, feestjes, roddels, reisjes en diners!

Mats en Lisa, wij gaan way back en hebben elkaar geloof ik ontmoet in de zandbak. Wij zijn alledrie intussen een compleet andere weg in geslagen, maar zijn nog steeds een heerlijk olijk trio. Bedankt voor de nodige ontspanning tijdens mooie feestjes in de zon!

Alle vrienden van de studenten alpenclub MaasSAC, bedankt voor de ontelbare momenten in de bergen, rotsen en heuvels. Deze afleiding was (en is) absoluut onmisbaar en heeft al vaak gezorgd voor een leeg en gezond hoofd. Oliver, Michael, Yuliya, Daphne, Hanneke, Chris, Pieter, Sabine, Sytze, Leah, Astrid, Matthijs, Irene en de rest: ik hoop dat we nog veel mooie tochten en Gipfelschnäpse zullen hebben samen.

Geneesco's van (onder andere) de eerste onderwijsgroep, bedankt voor de vele weekenden en feestjes die we over de jaren hebben gehad. Leonie, oude roomy, ik vind het bizar knap wat jij daar allemaal doet in Utrecht. Puck, wat is het tof dat wij gewoon even hetzelfde pad gaan bewandelen. Ik kijk er naar uit je weer in Nieuw Zeeland te zien!

Vele collega's van de huisartsenopleiding, bedankt voor de gezelligheid en de eindeloze momenten van reflectie, gelukkig vaak in de Thembi.

Juzer, we met years ago in Vancouver where you introduced me to science and taught me some professional Excel skills that later turned out to be vital. Your curiosity in life and reflective power inspires me. Thanks for the great conversations and your friendship.

Lieve familie, bedankt dat jullie mijn keuzes altijd steunen en trots op me zijn. Dankzij jullie heb ik geleerd nieuwsgierig, open en enthousiast te zijn. Pap, jij nam mij vroeger als klein meisje vaker mee naar jouw lab, waar ik met opengesperde ogen rondkeek en waardoor blijkbaar een vlammetje in me is aangewakkerd. Het was een eer enkele jaren geleden jouw paranimf te mogen zijn. Mam, het betekent veel voor me dat ook jij mijn plannen om te werken in een ver buitenland steunt, ook al weet ik dat je Maastricht eigenlijk al veel te ver weg vindt. Marleen, liefste zus, wij gaan door dik en dun en kennen elkaar door en door. Bovendien heb jij de twee knapste kinderen van de wereld: Hugo en Simon, durft te dromen, dat is het waard. Tim, ik ben trots dat jij mijn broer bent en ik weet dat we er altijd voor elkaar zijn! Opi en Omi, onze hechte familieband betekent heel veel voor mij, bedankt voor jullie steun. 
Liebe Schwiegerfamilie, danke, dass ich bei euch immer Wilkommen bin und Teil eurer lieben Familie sein darf.

Liefste Jan, jij bent mijn grootste supporter. Tijdens de pieken, maar ook de dalen die dit promotietraject heeft gekend bleef je altijd positief en in mij geloven. Een ding weet ik zeker, zonder jou was dit boekje nu niet hier. Bedankt voor je enthousiasme, geduld en liefde. Nu is het eindelijk tijd om het leven en de wereld samen verder te ontdekken. Ich liebe dich! 

Biography 



\section{Biography}

Gusta van Zwieten was born on the $14^{\text {th }}$ of August 1990 in Oostzaan, the Netherlands. After graduating secondary school (Blaise Pascal College, Zaandam), she moved to the South of the Netherlands to study Medicine at Maastricht University in 2008. In the final year of her Master's program, she completed her scientific internship on diagnostics in otolithic disorders in Vancouver General Hospital in Canada under supervision of dr. Art Mallinson and dr. Neil Longridge. Intrigued by research in the field of neuro-otological and often poorly understood pathologies, she started her PhD-project in November 2014, supervised by prof. dr. Yasin Temel, neurosurgeon and prof. dr. Robert J Stokroos, ENT physician.

Her research explores deep brain stimulation as a possible treatment for tinnitus and continues on the work of dr. Jasper V Smit. Gusta presented the results of the conducted studies at various international neuroscience and tinnitus conferences, in Cologne and Düsseldorf (Germany, 2015 and 2016), Łódź (Poland, 2016), Madrid (Spain, 2016), San Diego (USA, 2018), and Taipei (Taiwan, 2019).

After more than two years of full-time research in the Neuroscience Lab, she started the training to become a general practitioner in 2017, whilst continuing her research activities. In 2021, Gusta started working as a GP and is planning to fulfill a long-held dream and work as a rural doctor in New Zealand later in the same year. 

List of publications 



\section{List of publications}

van Zwieten G, Roberts MJ, Schaper FLVW, Smit JV, Temel Y, Janssen MLF. Noise-induced neurophysiological alterations in the rat medial geniculate body and thalamocortical desynchronization by deep brain stimulation. J Neurophysiol. 2021.

van Zwieten $G^{*}$, Zare $A^{*}$, Kotz SA, Temel Y, Schultz BG, Schwartze M, Janssen MLF. The effect of noise trauma and high-frequency stimulation on thalamic sensory gating in rodents. BioRxiv preprint. 2020.

Brunings JW, Vanbelle S, Hamaekers AEW, Kremer B, Bašić S, van Zwieten G, Baijens LWJ. Voice and vocal fold condition following short-term general anesthesia: a prospective study. J Voice. 2020.

van Zwieten $G_{\llcorner}$Jahanshahi A, van Erp M, Temel Y, Stokroos RJ, Janssen MLF, Smit JV. Alleviation of tinnitus with high frequency stimulation of the dorsal cochlear nucleus: a rodent study. Trends Hear. 2019.

Mallinson AI, Kuijpers ACM, van Zwieten G, Kakal J, Mullings WP, Longridge NS. Computerized Dynamic Posturography does not detect measured CVEMP and OVEMP abnormalities. Gait Posture. 2019.

van Zwieten G, Janssen MLF, Smit JV, Janssen AML, Roet M, Jahanshahi A, Stokroos RJ, Temel Y. Inhibition of Experimental Tinnitus With High Frequency Stimulation of the Rat Medial Geniculate Body. Neuromodulation. 2019.

van Zwieten G, Smit J, Jahanshahi A, Temel Y, Stokroos J. Tinnitus: is There a Place for Brain Stimulation? Surg Neurol Int. 2016.

van Zwieten $G^{*}$, Stuut $M^{*}$, Straetmans JM, Lacko $M$, Stumpel CT. The Inflatable Thymus - Herniation of the Normal Mediastinal Thymus: A Case Report and Review of the Literature. Int J Pediatr Otorhinolaryngol. 2016.

Smit J, Janssen M, van Zwieten G, Jahanshahi A, Temel Y, Stokroos J. Deep brain stimulation of the inferior colliculus in the rodent suppresses tinnitus. Brain Res. 2016. 
van Zwieten G, van Unen JMJ. Een man met een zwelling in de hand. Ned Tijdschr Geneeskd. 2014.

van Zwieten G, Goede de E, van der Laan R, Heemskerk J. A typical case of Bouveret's Syndrome, or Not? J Gastrointestin Liver Dis. 2014.

van Zwieten G, Devos JVP, Kotz SA, Ackermans L, Brinkmann P, Dauben L, George ELJ, Janssen AML, Kremer B, Leue C, Schwartze M, Temel Y, Smit JV, Janssen MLF. Deep brain stimulation for refractory tinnitus: a protocol for a randomised double-blinded cross-over clinical pilot study (submitted) 



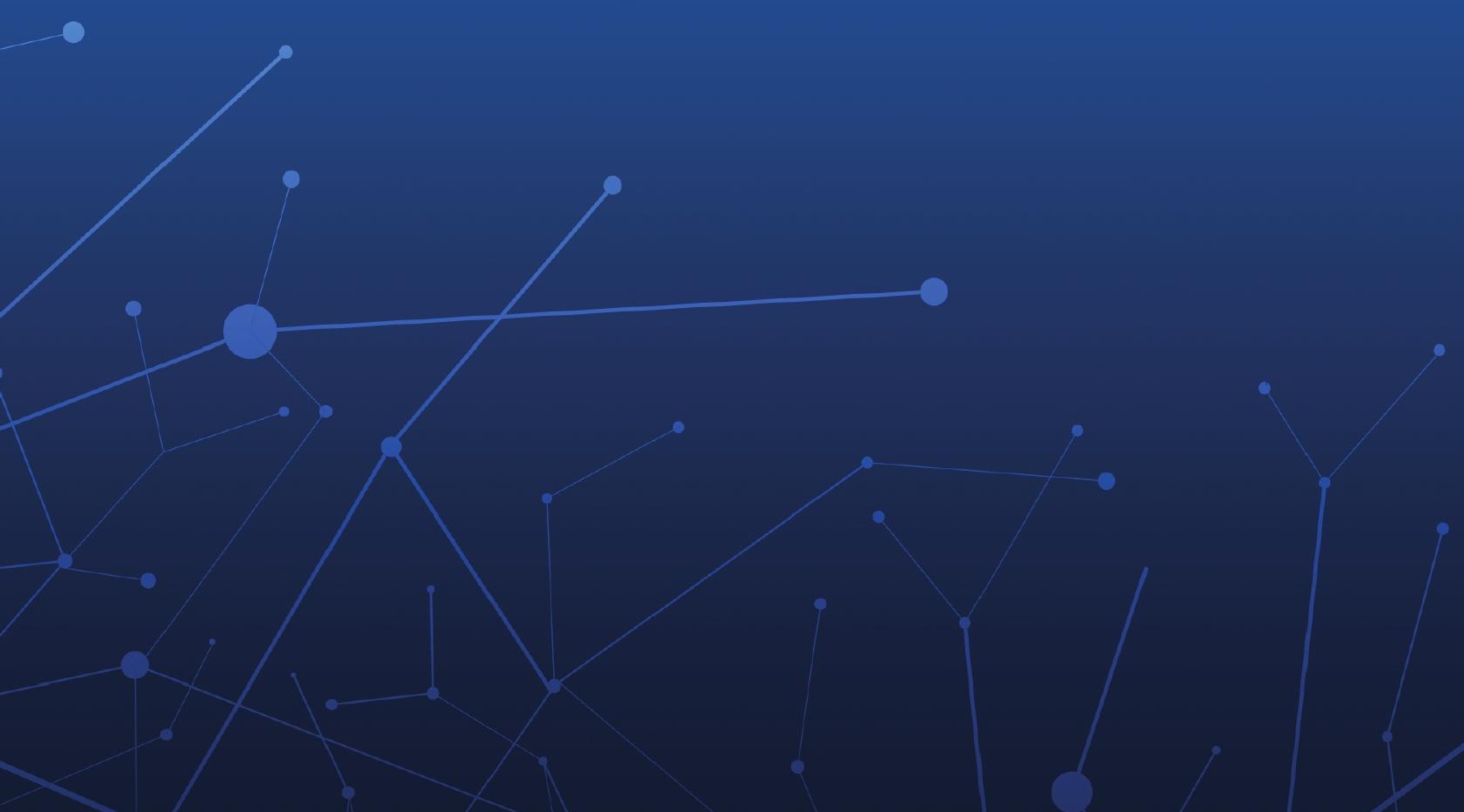

